\author{
Universidade de São Paulo \\ Instituto de Física
}

\title{
Ruídos quânticos da luz em macro cavidade de fibra óptica
}

\section{Renato Ribeiro Domeneguetti}

\author{
Orientador: Prof. Dr. Paulo A. Nussenzveig \\ Dissertação de mestrado apresentada ao \\ Instituto de Física para a obtenção do \\ título de Mestre em Ciências.
}

Banca Examinadora:

Prof. Dr. Paulo Alberto Nussenzveig (IFUSP)

Prof. Dr. Cristiano Luis Pinto de Oliveira (IFUSP)

Prof. Dr. Gustavo Wiederhecker (IFGW)

São Paulo 


\section{.}




\section{Agradecimentos}

Os meus mais sinceros agradecimentos ao meu orientador, o prof. Paulo A. Nussenzveig, e ao prof. Marcelo Martinelli, por terem me aceitado para fazer parte deste grupo de pesquisa tão importante. Foram dois anos de aprendizado muito importante na minha vida, tanto na parte profissional quanto na vida pessoal.

Sou grato ao prof. Hugo L. Fragnito, do laboratório de eletrônica quântica do Instituto de Física Gleb Wataghin da Universidade de Campinas (IFGW), por ter me cedido um espaço em seu laboratório para realizar o meu treinamento prático e executar a construção da cavidade de fibra óptica. Agradeço também pela utilização de seus materiais e equipamentos, e principalmente, por sua atenção e dedicação dispensada no desenvolvimento do projeto ao longo do período.

Sou grato ao prof. Gustavo Wiederhecker (IFGW), pelas excelentes discussões que tivemos sobre cavidades óticas, grande foi o meu avanço neste assunto.

Agradeço o prof. Paulo Dainese (IFGW), por diversas discussões sobre o comportamento do espalhamento Brillouin, tivemos muitas ideias de como podemos eliminar ou contornar este problema. A sua participação foi de fundamental importância no meu trabalho. 
Sou profundamente grato aos professores Christiano José Santiago de Matos e Eunézio Antônio de Souza (Thoróh) da Universidade Mackenzie, pelas primeiras discussões sobre o meu projeto. Foi uma grande satisfação poder visitar o laboratório de fotônica da universidade, e conhecer as pesquisas que por eles estão sendo desenvolvidas.

Obrigado aos professores Flavio C. Cruz e Luís Eduardo E. de Araujo (IFGW), pelo aprendizado adquirido durante minha estadia na Unicamp. Com eles aprendi bastante sobre eletrônica e sistemas de travamento em geral.

Sou igualmente grato aos técnicos do laboratório de Fibras Ópticas do professor Hugo, Wagner e Paulo, pela constante dedicação em minha familiarização com o manuseio de fibras ópticas e equipamentos eletrônicos.

Ao aluno de doutorado do prof. Hugo, Alexander Ramirez, pelo treinamento com fibras.

Agradeço a CNPq pelo apoio financeiro.

Em especial, gostaria de agradecer a todo o pessoal do LMCAL, todos tiveram uma participação importante neste perído. Ao Antônio, Felippe, Renné, Igor, Paula, Hans, Flávio, Klara, Rayssa, Carlos, Ashok, e todos outros que tive contato.

À Deus e à minha família. Não teria conseguido chegar até aqui sem a ajuda deles. 


\section{Resumo}

Mistura de quatro ondas não degenerada em fibras ópticas na configuração de cavidade foi inicialmente proposto como forma de geração de estados comprimidos da luz. Neste trabalho desenvolvemos um tratamento puramente quântico da análise da interação não linear entre a luz e a matéria. Com a equação de Fokker-Planck na representação de Wigner obtemos diretamente das equações o limiar de oscilação dos feixes gêmeos e o efeito de biestabilidade. Das equações de dinâmica linearizadas para as flutuações de quadraturas confirmamos a geração de estados comprimidos provenientes do processo de 4WM, tanto abaixo quanto acima do limiar de oscilação. Não é possível iniciar a geração dos feixes gêmeos sem antes alcançar o limiar de oscilação do espalhamento Brillouin estimulado. Portanto, técnicas para aumentar este limiar devem ser empregadas em qualquer medida de ruído ao nível de shot-noise com geração de campos. Nós também testamos as limitações técnicas intrínsecas do laser de diodo, como o ruído de amplitude e fase, assim como sua estabilidade em um experimento interferômetrico. Abaixo do limiar, a cavidade atua no sentido de reduzir o ruído de fase do laser em um intervalo de frequência que vai de $10 \mathrm{MHz}$ a $80 \mathrm{MHz}$. 


$$
\text { . }
$$




\begin{abstract}
Nondegenarate four-wave mixing in an optical-fiber cavity geometry, was initially proposed as a mean to generate squeezed states of light. We developed in this work a purely quantum analyzis of the nonlinear interaction between light and medium. With Fokker-Planck equation in the Wigner representation, we obtained directly from the equations, the twin beams oscillation threshold and bistability. From the linearized dynamic equations for the quadratures fluctuations, we confirmed the generation of squeezed states proceeding from $4 \mathrm{WM}$ process, not only below but above the threshold oscillation. It is not possible to initiate the twin beams generation without first reaching the stimulated Brillouin scattering threshold. Therefore, techniques to increase this threshold, must be used for any noise measure to the shot-noise level with fields generation. We also have tested the technical limitations of laser diode, as amplitude and phase noise, as well as the stability in an interferometric experiment. Below threshold, the cavity acts in the sense of reducing the intrinsic laser phase noise in a frequency range that goes from $10 \mathrm{MHz}$ to $80 \mathrm{MHz}$.
\end{abstract}




$$
\text { . }
$$


Dedicado à minha mãe e ao meu irmão, Safira e Fernando. 



\section{Conteúdo}

$\begin{array}{ll}\text { Lista de siglas } & 13\end{array}$

1 Introdução 15

2 Descrição do campo eletromagnético 19

2.1 Descrição clássica . . . . . . . . . . . . . . . . . . . . . . . . . . . . . . . . 19

2.2 Medida do campo eletromagnético . . . . . . . . . . . . . . . . 22

2.3 Estados quânticos do campo . . . . . . . . . . . . . . . . . . . . . . . . . . . . . . . . . . . 24

2.3.1 Estados coerentes . . . . . . . . . . . . . . . . . . 26

2.3 .2 Estados comprimidos . . . . . . . . . . . . . . . . . 27

2.4 Medida da função de Wigner . . . . . . . . . . . . . . . . . . . . . . 30

2.5 Medidas das variâncias . . . . . . . . . . . . . . . . . . . . . . . . 33

2.6 Calibração de shot-noise . . . . . . . . . . . . . . . . . . . . . 40

3 Fibras ópticas $\quad 45$

3.1 Introdução . . . . . . . . . . . . . . . . . . . . . . 45

3.2 A Hamiltoniana não linear . . . . . . . . . . . . . . . . . . . . . . . . . . 49

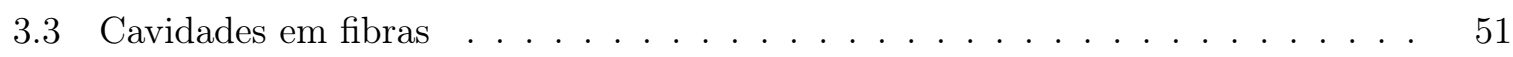

3.4 Efeito da cavidade . . . . . . . . . . . . . . . . . . . 57

3.5 Mistura de quatro ondas . . . . . . . . . . . . . . . . . . 62

4 Compressão em fibras $\quad 67$

4.1 Tratamento estocástico . . . . . . . . . . . . . . . . . . . . . 67

4.2 Representação de Wigner . . . . . . . . . . . . . . . . . . . . 72

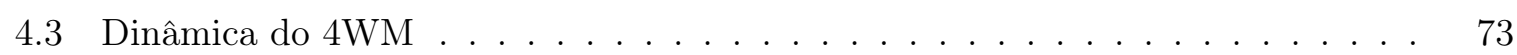

4.4 Solução estacionária . . . . . . . . . . . . . . . . . . . . . 77

4.5 Equações linearizadas . . . . . . . . . . . . . . . . . . . . . . . 79

4.6 Espectro de flutuações $\ldots \ldots \ldots$. . . . . . . . . . . . . . . . . . . 82 
4.7 Espectro de compressão . . . . . . . . . . . . . . . . . . . . 84

4.7 .1 Acima do limiar de oscilação . . . . . . . . . . . . . . . . . . 85

4.7 .2 Abaixo do limiar de oscilação . . . . . . . . . . . . . . . . . . 86

5 Descrição Experimental $\quad 91$

5.1 Espalhamento Brillouin . . . . . . . . . . . . . . . . . . . . . . . 91

5.1.1 Espalhamento Brillouin estimulado . . . . . . . . . . . . . . . . . 91

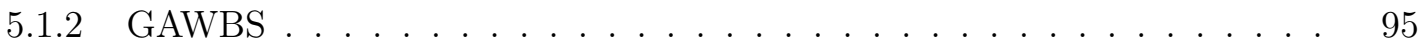

5.2 Caracterização instrumental . . . . . . . . . . . . . . . . . 96

5.3 Aparato Experimental . . . . . . . . . . . . . . . . . . . . . . . . 100

5.4 Primeiras análises . . . . . . . . . . . . . . . . . . . . . 103

5.4 .1 Efeito cavidade . . . . . . . . . . . . . . . . . 105

5.5 Medidas de ruído . . . . . . . . . . . . . . . . . . . . . 107

6. Conclusões e perspectivas 111

A Sistema de travamento 113 


\section{Lista de siglas}

\begin{tabular}{lll} 
Sigla & Inglês & Português \\
\hline OPA & Optical Parametric Amplifier & Amplificador paramétrico óptico \\
OPO & Optical Parametric Oscillator & Oscilador paramétrico óptico \\
4WM & Four Wave Mixing & Mistura de quatro ondas \\
FSR & Free Spectral Range & Intervalo espectral livre \\
HNLF & Highly Non-Linear Fiber & Fibra altamente não linear \\
BS & Beam Splitter & Divisor de feixe \\
PBS & Polarization Beam Splitter & Divisor de feixe por polarização \\
DC & Direct Current & Corrente contínua \\
HF & High frequency & Sinal de alta frequência \\
PZT & Piezoeletric Actuator & Atuador piezoelétrico \\
ZDW & Zero Dispersion Wavelength & Comprimento em zero de dispersão \\
QND & Quantum Nondemolition & Não demolição quântica \\
SBS & Stimulated Brillouin Scattering & Espalhamento Brillouin estimulado \\
GAWBS & Guided Acoustic Wave - & Espalhamento Brillouin guiado - \\
& Brillouin Scattering & por ondas acústicas \\
CW & Continuous Wave & Feixe contínuo \\
SQL & Standart Quantum Limit & Limite quântico padrão \\
FWHM & Full Width at Half Maximum & Largura à meia altura \\
CMOS & Complementary Metal-Oxide- & Semicondutor metal-óxido \\
& Semiconductor & complementar
\end{tabular}




\section{.}




\section{Capítulo 1}

\section{Introdução}

Desde a invenção do telefone, Alexander Graham Bell pensou em utilizar a luz como meio de comunicação. A dificuldade é que até antes de 1960 não existia uma fonte capaz de produzir luz coerente, isto é, com uma frequência e direção de propagação bem definidas. A invenção do laser possibilitou a retomada da pesquisa nessa área. Os pesquisadores começaram a ver o espectro de luz como uma extensão da radio frequência.

Atualmente, sistemas de comunicação óptica tem sido desenvolvido por grandes empresas, com uso militar, espacial e civil. O laser produz luz coerente com frequência de portadora da ordem de $10^{15} \mathrm{~Hz}$. Uma largura de banda de $0,1 \%$ representa uma largura de banda de informação de 1000GHz. A vantagem desse sistema reside na possibilidade de transmitir através de um mesmo caminho óptico múltiplas informações.

Pesquisas estão sendo desenvolvidas na tentativa de implementar essa tecnologia em sistemas integrados. O objetivo é aplicar a tecnologia de circuitos integrados, para produzir sistemas ópticos na escala micrométrica. Com essas tecnologias, podemos imaginar alternativas para os circuitos elétricos, em sistemas que operam com luz coerente [29].

As fibras ópticas são guias de ondas que atuam no comprimento de onda do infravermelho próximo, $1,55 \mu \mathrm{m}$, conhecido como telecom. Sua estrutura é constituída de um núcleo de vidro e um entorno com índice de refração levemente inferior, aproximadamente 1\%. Para conseguir essa pequena diferença no índice de refração, o núcleo e o entorno são fabricados com o mesmo material, mas o núcleo é dopado tipicamente com germânio [35]. As primeiras fibras ópticas tinham uma taxa de perda de $20 \mathrm{~dB} / \mathrm{km}$, sendo necessário a instalação de amplificadores eletrônicos, conhecidos como repetidores, a cada $3 \mathrm{~km}$. O alto custo da instalação desses sistemas acabaram tornando inviáveis a implementação das fibras como guias de ondas.

Com o avanço da técnica aplicada na construção das fibras, atualmente é possível construí-las 
com perdas da ordem de $0,5 \mathrm{~dB} / \mathrm{km}$. Com as perdas reduzidas, a necessidade de instalações de amplificadores passaram a ser a cada 100km. Hoje, devido aos grandes esforços empregados na área de pesquisa sobre efeitos não lineares em fibras, os amplificadores eletrônicos foram substituídos por amplificadores ópticos.

Os amplificadores ópticos são sistemas que amplificam o sinal da fibra diretamente, sem a necessidade de converter o sinal óptico em sinal eletrônico. Existem várias técnicas utilizadas para realizar a amplificação diretamente do sinal óptico, técnicas como amplificação por efeito Raman em fibras [50], dopagem da fibra em certos pontos com Erbium [38], e também os amplificadores paramétricos ópticos (OPA). Estes fazem uso do termo de não linearidade óptica das fibras, um ganho devido a conversões paramétricas entre campos acoplados.

Interações por processos paramétricos em um meio não linear podem levar à geração e amplificação de novos campos em detrimento da atenuação de outros. Devido às possibilidades de aplicações práticas dos campos amplificados, uma maior atenção ao processo de geração e suas propriedades foram dispensadas a eles. Contudo, a atenuação dos campos responsáveis pelas amplificações está por trás do processo de geração de estados comprimidos da luz [54].

Primeiramente, os estados comprimidos foram descritos teoricamente, e desde então, vários experimentos foram propostos e realizados na tentativa de demonstrarem sua existência $[5,25]$. Contudo, a geração de estados comprimidos não é uma realização trivial. Do ponto de vista experimental, a geração destes estados requer que várias dificuldades sejam contornadas [28]. O campo eletromagnético pode ser discutido de duas maneiras, ou mais especificamente, através de dois tratamentos, o tratamento clássico e o quântico. No tratamento clássico, o campo eletromagnético é tratado como um campo elétrico que oscila no tempo $E(t)=E_{1} \sin (\omega t)+E_{2} \cos (\omega t)$, onde $E_{1}$ e $E_{2}$ são ditos as quadraturas do campo. Uma forma de representação destas quantidades é por meio do espaço de fase, e cada estado do campo pode ser representado neste mapa. Classicamente, cada uma das quadraturas pode ser medida com precisão infinita, representado como um ponto no espaço de fase.

Na mecânica quântica, as quadraturas passam a serem consideradas como variáveis canônicas conjugadas, que estão sujeitas ao princípio da incerteza de Heisenberg. Com isso, o estado do campo deixa de ser representado por um ponto e passa para uma representação indicada por um valor médio mais uma densidade de probabilidade em torno deste, que reflete os possíveis valores das quadraturas ditadas pelo princípio da incerteza [40]. O estado coerente por exemplo, é representado por um valor médio mais um contorno circular em torno deste ponto, representando sua incerteza na medida. O raio deste contorno, indica que a variância nas duas quadraturas são iguais é obedecem ao limite mínimo dado pelo princípio da incerteza. Estados comprimidos por sua vez, são estados do campo que admitem a redução da variância em uma de suas quadraturas para um valor inferior ao do estado coerente. Sua representação no espaço 
de fase é uma elipse, com eixo menor indicando a quadratura reduzida. A incerteza na medida das quadraturas do campo é relacionada com as flutuações de vácuo. Portanto, um processo não linear que possibilita a geração de estados comprimidos, é uma ferramenta que permite a alteração das flutuações de vácuo para valores inferiores aos limitados pelo princípio da incerteza de Heisenberg.

A medição do campo eletromagnético exige um sistema de deteç̧ão capaz de medir ambas as quadraturas, amplitude e fase. A deteç̧ão simples da luz mede o fluxo de fótons que atinge o detector, que é proporcional à quadratura de amplitude. Técnicas de interferência na detecção devem ser implementadas com o objetivo de medir a quadratura de fase. A técnica de detecção homodina emprega o batimento entre um campo intenso, denominado de oscilador local, e o campo de interesse. Ambos os campos devem possuir uma relação bem definida de frequência e fase, que combinados em um beam-splitter e colimados em um fotodiodo, a mecânica quântica prediz que as pequenas flutuações de amplitude e fase do campo de interesse correspondem às flutuações do oscilador local [57, 41]. O uso da técnica de cavidades, aproveita o caráter dispersivo dos ressoadores para induzir uma diferença de fase entre o oscilador local, neste caso a portadora, e as flutuações de vácuo, tratadas como bandas laterais [26, 53].

Para a observação de estados comprimidos é necessário primeiramente uma fonte de luz de estados coerentes. Os lasers costumam apresentar excesso de ruído em uma das quadraturas. Para lasers não muito ruidosos, através das cavidades de filtro podemos filtrar este excesso de ruídos a níveis próximos do shot-noise. Outra alternativa é trabalhar em regiões de alta frequência, onde normalmente o excesso de ruído deixa de existir.

A Hamiltoniana de interação responsável pela geração de estados comprimidos é quadrática nos operadores de criação e aniquilação da mecânica quântica, ou é bilinear em modos distintos. Ela é diferente da Hamiltoniana dos estados coerentes, que é linear nos operadores de criação e aniquilação. O processo não linear aplicado aqui é a mistura de quatro ondas. As interações paramétricas acoplam dois fótons gerados, sinal e complemetar, com dois fótons do campo de bombeio. A aniquilação simultânea de dois fótons do bombeio para a geração também simultânea dos fótons sinal e complementar é um indício da existência de propriedades não quânticas da luz, como compressão e emaranhamento. Se os modos dos fótons gerados não forem em número grande o suficiente para definir um valor médio do campo, estes serão tratados como flutuações do vácuo.

A principal dificuldade experimental neste processo reside no fato de que a interação óptica não linear responsável pela geração de estados comprimidos é muito fraca, enquanto que, efeitos indesejáveis como atenuação, difração e espalhamento, podem ser bastante significativos. A primeira proposta para contornar estas dificuldades foi a realização de mistura e quatro ondas em fibras ópticas [26], como forma de aumentar espacialmente a interação entre a luz e o meio 
não linear. Outra alternativa, neste caso estratégica, foi a utilização de cavidade como forma de intensificar os processos não lineares $[58,47,8]$. Seguindo estas direções, nada mais razoável do que pensar em unir as duas técnicas para construir uma cavidade com fibras ópticas [43]. Iremos seguir aqui esta mesma proposta levando em conta que, com as novas tecnologias desenvolvidas na produção de fibras óticas, a atenuação dos campos nas fibras foram reduzidas a um nível que podemos construir cavidades maiores e com perdas equivalentes. O avanço tecnológico também permitiu a construção de fibras com núcleo menores, aumentando o fator de não linearidade da fibra. Com o desenvolvimento de novos lasers, principalmente na faixa de $1550 \mathrm{~nm}$, onde ocorre menos absorção pela fibra, estes ficaram mais estáveis e menos ruidosos, condição favorável para verificar um nível de compressão nas quadraturas mais significativo. 


\section{Capítulo 2}

\section{Descrição do campo eletromagnético}

Iniciaremos com a descrição clássica do campo eletromagnético, definindo as funções de correlação clássicas do campo. Seguiremos com a explicação de como podemos medir o campo eletromagnético, e interpretar as flutuações na corrente elétrica como provenientes das flutuações do campo eletromagnético. Faremos também um tratamento quântico do campo, sendo um pouco diferente do que é costumado a ser feito, através dos auto estados da Hamiltoniana. Seguiremos a descrição em termos da função de quasiprobabilidade de Wigner, processo que se assemelha à descrição feita na mecânica clássica. Por fim, explicaremos a técnica de detecção utilizada neste trabalho, a detecção por cavidades.

\subsection{Descrição clássica}

O campo eletromagnético clássico entendido como procedente das equações de Maxwell [22] é descrito por uma função real $\alpha^{(r)}(\mathbf{r}, t)$, que caracteriza o campo no ponto $\mathbf{r}$ e no tempo $t$. Ela pode representar por exemplo o campo elétrico, o campo magnético ou o potencial vetor. Em geral não especificamos a natureza física que a função descreve, e a principal razão para isso é que a descrição é independente de uma escolha particular da grandeza clássica. No entanto, uma vez determinado o caráter particular do problema, é conveniente a priori, determinar a natureza da grandeza física, pois com a escolha correta podemos muitas vezes simplificar a abordagem.

A função $\alpha^{(r)}(\mathbf{r}, t)$ flutua no tempo, e a causa principal de sua flutuação é estabelecida em sua origem. A flutuação temporal pode ser entendida como uma assinatura do campo contendo informações a respeito de todos as componentes de frequência que a gerou. Por exemplo, a luz 
gerada por uma fonte térmica contém componentes em frequências aleatórias e independentes umas das outras. Neste caso o campo é dito incoerente e suas flutuações são tratadas estatisticamente [18]. Por outro lado, a luz gerada por uma fonte coerente, como o feixe monocromático de um laser, possui um espectro estreito de flutuações. Mas apesar disto, ainda existem flutuações aleatórias causadas principalmente pela emissão espontânea e questões técnicas diversas, como por exemplo as vibrações dos espelhos que compõem a cavidade interna do laser.

Com estas considerações, é intuitivo tentar descrever o campo eletromagnético como sendo composto por um espectro de frequências, cujo efeito global é produzir o sinal temporal em questão. Assumimos que a decomposição se dá no espaço de Fourier [33]

$$
\alpha^{(r)}(\mathbf{r}, t)=\int_{-\infty}^{\infty} \alpha(\mathbf{r}, \nu) \exp (-2 \pi i \nu t) d \nu
$$

e notamos que $\alpha^{*}(\mathbf{r}, \nu)=\alpha(\mathbf{r},-\nu)$. Por essa relação percebemos que toda a informação existente no lado negativo do espectro está contida na parte conjugada do espectro positivo, portanto é oportuno definir a função complexa

$$
\alpha(\mathbf{r}, t)=\int_{0}^{\infty} \alpha(\mathbf{r}, \nu) \exp (-2 \pi i \nu t) d \nu
$$

Uma propriedade importante que surge da descrição clássica reside no efeito da coerência, que essencialmente retrata a manifestação de correlações entre flutuações de campos separados no espaço tempo. Sendo assim, podemos definir a função de correlação entre campos distintos. Para isso, imaginamos duas fontes pontuais de luz localizadas em dois pontos distintos $\mathbf{r}_{1}$ e $\mathbf{r}_{2}{ }^{1}$. Em um ponto $\mathcal{P}$ genérico, com coordenada $\mathbf{r}$ e distanciado de $s_{1}$ e $s_{2}$ com relação a $\mathbf{r}_{\mathbf{1}}$ e $\mathbf{r}_{\mathbf{2}}$ respectivamente, aceitamos a existência de um campo complexo dado por

$$
\alpha(\mathbf{r}, t)=K_{1} \alpha\left(\mathbf{r}_{1}, t-t_{1}\right)+K_{2} \alpha\left(\mathbf{r}_{2}, t-t_{2}\right)
$$

onde $t_{1}=s_{1} / c$ e $t_{2}=s_{2} / c$ é o tempo em que a luz leva para se propagar dos pontos $\mathbf{r}_{1}$ e $\mathbf{r}_{2}$, respectivamente, e chegar ao ponto $\mathcal{P}$. $K_{1}$ juntamente com $K_{2}$ são constantes que contabilizam a estrutura da fonte de luz, como sua natureza e geometria, nos pontos $\mathbf{r}_{1}$ e $\mathbf{r}_{2}$ respectivamente. Um fotodetector colocado em $\mathbf{r}$ irá medir a intensidade do campo ${ }^{2}$

$$
I(\mathbf{r}, t)=\alpha^{*}(\mathbf{r}, t) \alpha(\mathbf{r}, t),
$$

\footnotetext{
${ }^{1} \mathrm{Na}$ prática, não existe fontes pontuais de luz. Experimentalmente podemos nos aproximar da situação ideal fazendo um feixe de luz atravessar um orifício pequeno (pinhole), e fazer a análise dos campos após este ponto.

${ }^{2}$ De fato, é possível associar a função complexa $\alpha(\mathbf{r}, t)$ com o operador de aniquilação da mecânica quântica que, na teoria de fotodeteç̧ão, está diretamente relacionado com a medida de intensidade do campo [17], como veremos mais à frente.
} 
e medindo por um tempo maior que o tempo de coerência temos

$$
\langle I(\mathbf{r}, t)\rangle=\left|K_{1}\right|^{2} I_{1}\left(\mathbf{r}_{1}, t-t_{1}\right)+\left|K_{2}\right|^{2} I_{2}\left(\mathbf{r}_{\mathbf{2}}, t-t_{2}\right)+2 \Re\left[K_{1}^{*} K_{2} \Gamma\left(\mathbf{r}_{1}, \mathbf{r}_{2}, t-t_{1}, t-t_{2}\right)\right],
$$

onde $\Re$ se refere à parte real do termo e

$$
\Gamma\left(\mathbf{r}_{1}, \mathbf{r}_{2}, t_{1}, t_{2}\right)=\left\langle\alpha^{*}\left(\mathbf{r}_{1}, t_{1}\right) \alpha\left(\mathbf{r}_{2}, t_{2}\right)\right\rangle
$$

A expressão (2.1.6) representa a correlação temporal entre os dois campos. Fazendo uso da notação, definimos a intensidade média dos campos individuais no ponto $\mathbf{r}_{\imath}$ como $\Gamma\left(\mathbf{r}_{\imath}, \mathbf{r}_{\imath}, t_{\imath}, t_{\imath}\right)$, com $\imath=1$ ou 2 .

Normalmente consideramos a descrição do campo eletromagnético clássico no regime estacionário. Sob esta condição, a função que descreve o campo perde qualquer informação a respeito da origem dos tempos e, em qualquer correlação existente, o argumento temporal da função de correlação irá depender apenas da diferença dos tempos $t_{2}-t_{1}$. Iremos discutir um pouco mais sobre a estacionariedade na próxima seção.

Notamos pela expressão (2.1.5), que a intensidade em $\mathbf{r}$ não é igual à soma das intensidades nos pontos $\mathbf{r}_{1}$ e $\mathbf{r}_{2}$. Elas diferem por $2 \Re\left[K_{1}^{*} K_{2} \Gamma\left(\mathbf{r}_{1}, \mathbf{r}_{2}, t_{2}-t_{1}\right)\right]$, responsável pelo efeito de interferência.

É conveniente normalizar a função de correlação pelas intensidades individuais

$$
C\left(\mathbf{r}_{1}, \mathbf{r}_{2}, \tau\right)=\frac{\Gamma\left(\mathbf{r}_{1}, \mathbf{r}_{2}, \tau\right)}{\left[\Gamma\left(\mathbf{r}_{1}, \mathbf{r}_{1}, 0\right)\right]^{1 / 2}\left[\Gamma\left(\mathbf{r}_{2}, \mathbf{r}_{2}, 0\right)\right]^{1 / 2}} .
$$

Da desigualdade de Schwarz é possível mostrar que

$$
0 \leq\left|C\left(\mathbf{r}_{1}, \mathbf{r}_{2}, \tau\right)\right| \leq 1
$$

A noção de coerência do eletromagnetismo clássico denota a tendência dos resultados de duas medidas realizadas, espacialmente separadas ou temporalmente distantes, estarem correlacionados. O efeito de interferência observado num ponto é um indício da existência de correlação, como observado na discussão acima.

A definição de coerência aqui apresentada está direcionada à correlação de intensidade, que pode ser medida por um fotodetector. Para este tipo de correlação, a expressão (2.1.7) completa a noção de coerência do campo eletromagnético clássico.

Com o surgimento do laser, a idéia de coerência passou a ser considerada como uma característica intrínseca a tais objetos, associando esta propriedade com perfil estreito de seu espectro. No entanto, com o advento da óptica não linear, envolvendo a interação da radiação com a 
matéria, experimentos levaram a observações de correlações de ordem superior em dois ou mais pontos separados no espaço tempo. Em resposta a estas observações, houve a necessidade de reformular a noção de coerência para uma idéia mais ampla [17], e sua concepção deixou de ser atribuída à largura de linha do espectro do laser e passou a ser considerada como uma qualidade do grau de organização tanto da radiação quanto da matéria.

\subsection{Medida do campo eletromagnético}

Experiências relacionadas com a luz, independente da forma de como ela é tratada, exigem normalmente medições do campo eletromagnético. Tal medida requer a utilização de fotodetectores, os quais utilizam o efeito fotoelétrico para produzirem uma fotocorrente $i(t)$. Estamos interessados inicialmente em campos intensos, os quais geram uma corrente nos fotodetectores. Acompanhado do valor médio da fotocorrente, medimos também suas flutuações, que representam os ruídos intrínsecos da luz e também as flutuações do detector. Uma descrição completa do ruído da fotocorrente é feita a partir da função de correlação, definida como em [12]

$$
C_{i}\left(t, t^{\prime}\right)=\left\langle i(t) i\left(t^{\prime}\right)\right\rangle-\langle i(t)\rangle\left\langle i\left(t^{\prime}\right)\right\rangle
$$

Na configuração de feixes intensos, a corrente gerada pelo fotodetector é estacionária. A implicação disto, é que a função de correlação será independente do instante inicial da primeira medida, dependendo apenas do intervalo temporal em que as duas medidas foram realizadas $t-t^{\prime}=\tau$.

Pode ser útil realizar a medida de ruído em uma frequência de análise específica. Como veremos adiante, qualquer medida geral de ruído pode ser feita por um equipamento conhecido como analisador de espectro, que mede o ruído da fotocorrente na frequência desejada. Por esse motivo é conveniente definirmos a transformada de Fourier da função de correlação, conhecida como densidade de ruído espectral

$$
S_{i}(\Omega)=\int_{-\infty}^{\infty} C_{i}\left(t, t^{\prime}\right) \exp (i \Omega \tau) d \tau
$$

Podemos definir a flutuação na corrente como $\delta i(t)=i(t)-\langle i(t)\rangle$. Da mesma maneira, definimos a flutuação de corrente espectral como

$$
\delta i(\Omega)=\int_{-\infty}^{\infty} \delta i(t) \exp (i \Omega t) d t \quad ; \quad \delta i(t)=\int_{-\infty}^{\infty} \delta i(\Omega) \exp (-i \Omega t) \frac{d \Omega}{2 \pi} .
$$


Por substituição direta mostra-se que

$$
\left\langle\delta i(\Omega) \delta i\left(\Omega^{\prime}\right)^{*}\right\rangle=2 \pi \delta\left(\Omega-\Omega^{\prime}\right) S_{i}(\Omega) .
$$

A expressão acima é um caso particular de um teorema mais geral, conhecido como teorema de Wiener Khinchine. A variância pode ser calculada fazendo $\tau=0$,

$$
(\Delta i)^{2}=C_{i}(\tau=0)=\int_{-\infty}^{\infty} S_{i}(\Omega) \frac{d \Omega}{2 \pi}
$$

A densidade de ruído espectral pode ser independente de $\Omega$, neste caso ela possui o mesmo valor para qualquer banda de frequência analisada e se espalha ao longo de todo o espectro. Sendo assim, sua variância é divergente, característica descrita como "ruído branco".

A medida da variância da corrente é realizada por equipamentos que calculam de forma eletrônica a integração do lado direito da (2.2.5). Em geral, o sistema completo de detecção é composto por um detector mais uma eletrônica que, entre outras funções, é capaz de filtrar a flutuação de corrente em um determinado intervalo de frequência. Portanto, a flutuação de corrente gerada pelo fotodiodo, que idealmente representa a flutuação do campo eletromagnético medido, é filtrada pela eletrônica de detecção, $\delta i_{F}(\Omega)=f(\Omega) \delta i(\Omega)$. Assim, a variância medida não representa a variância real da fotocorrente, o filtro espectral deve ser levado em conta

$$
\left(\Delta i_{F}\right)^{2}=\int_{-\infty}^{\infty}|f(\Omega)|^{2} S_{i}(\Omega) \frac{d \Omega}{2 \pi}
$$

É conveniente, como veremos, medir a variância em um intervalo estreito de frequência. Idealmente estamos interessados na medida da variância em uma frequência específica $\Omega_{0}$. O analisador de espectro é constituído por um filtro eletrônico de banda estreita, que de forma ideal se assemelha a uma delta de Dirac. Este filtro pode ser descrito por $|f(\Omega)|^{2}=2(2 \pi \delta f) \delta\left(\Omega_{0}\right)$, onde $\delta f$ é a largura de banda do filtro, assim a variância é escrita como

$$
\left(\Delta i_{F}\right)^{2}=2 \delta f S_{i}(\Omega)
$$

Portanto o analisador de espectro, através da medida da flutuação da corrente e das propriedades de seu filtro, permite uma medida real da densidade de ruído espectral.

Fizemos uma aplicação direta do teorema de Wiener Khinchine para as flutuações da corrente, que é a grandeza diretamente medida em experimentos de óptica, mas a validade do teorema se estende para qualquer tipo de flutuação da variável a ser definida. A condição que deve ser satisfeita é que não haja um ponto especial no tempo, isto é, que o valor da função de correlação não dependa do instante em que foi calculada, mas deve depender apenas da diferença entre os 
dois tempos. Uma situação como esta é tratada como um processo de Markov [23]. Uma forma bastante útil de se escrever o teorema de Wiener Khinchine é

$$
\left\langle\delta X(\Omega) \delta X\left(-\Omega^{\prime}\right)\right\rangle=2 \pi \delta\left(\Omega-\Omega^{\prime}\right) S_{X}(\Omega)
$$

o qual diz que a densidade de ruído espectral é proporcional à transformada de Fourier da função de correlação da variável $X(t)$. Mais adiante iremos discutir um pouco mais sobre este teorema.

\subsection{Estados quânticos do campo}

Classicamente, vimos que podemos descrever o estado do campo eletromagnético através de uma função de onda complexa definida como a transformada de Fourier de uma função definida no espaço de frequências. Toda informação contida no espectro de frequências está codificada na função de onda temporal, e obtemos estas informações acessando os componentes através da transformada de Fourier inversa, realizada pelo analisador de espectro.

No contexto da mecânica quântica, o estado de um sistema é muitas vezes representado em termos do auto-estado da Hamiltoniana $|\psi\rangle$, ou do operador densidade $\hat{\rho}$ [7]. Por meio destas entidades, é possível descrever completamente o estado quântico do campo ou da partícula, obter resultados de problemas, e entender as propriedades quânticas do sistema. No entanto, há outra representação da mecânica quântica que possibilita estudá-la de um modo que, em certos aspectos, se assemelha à maneira que estudamos a física clássica. Tal descrição é feita no

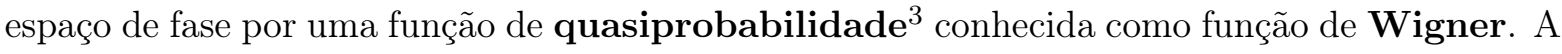
principal vantagem deste tratamento é atribuída à semelhança direta com a mecânica clássica, no que diz respeito à existência de função que representa, em termos das variáveis do sistema, uma distribuição de probabilidade, responsável por conter toda a informação do sistema. Neste cenário, a função de Wigner contém toda a informação, em caráter quântico, sobre o sistema, e podemos reconstruir a função através de medidas distintas do sistema, em um processo chamado de tomografia quântica [9].

Sabemos da quantização do campo eletromagnético que podemos pensar em cada modo do campo como um oscilador harmônico. Portanto, irei descrever nesta seção o tratamento dado ao oscilador harmônico em termos da distribuição de probabilidade de Wigner. Este conteúdo foi fortemente influenciado pela descrição feita em [40].

\footnotetext{
${ }^{3}$ É denominada de função de quasiprobabilidade por satisfazer propriedades semelhantes às quais as funções de probabilidade satisfazem, com exceção de que a função de Wigner pode assumir valores negativos.
} 
Na mecânica quântica, a informação sobre a probabilidade de encontrar o oscilador harmônico no estado $x$ está escrita em sua função de onda $\psi(x)$. E o módulo ao quadrado de $\psi(x)$ representa, em certo sentido, uma distribuição clássica de probabilidade dos estados do oscilador harmônico no espaço de posição. Podemos passar para a representação no espaço de momento pela transformada de Fourier da função de onda no espaço de posição, convenientemente normalizada

$$
\tilde{\psi}(p)=\int_{-\infty}^{\infty} \frac{d x}{\sqrt{2 \pi \hbar}} \exp \left(-i \frac{p}{\hbar} x\right) \psi(x)
$$

onde agora o módulo ao quadrado da $\tilde{\psi}(p)$, representa a probabilidade de encontrar o oscilador harmônico com um determinado momento $p$. Calculando o módulo ao quadrado desta função observamos que o argumento da exponencial depende apenas da diferença $x_{2}-x_{1}$ das posições. É conveniente então passarmos a descrição para um novo sistema de referência, cujas coordenadas representam agora a média e a diferença entre as posições, $x_{1}=x+y / 2$ e $x_{2}=x-y / 2$. Com esta mudança obtemos

$$
|\tilde{\psi}(p)|^{2}=\int_{-\infty}^{\infty} \frac{d x d y}{2 \pi \hbar} \exp \left(-i \frac{p}{\hbar} y\right) \psi(x+y / 2) \psi^{*}(x-y / 2) .
$$

Supondo hipoteticamente que $y$ seja zero, o produto $\psi(x+y / 2) \psi^{*}(x-y / 2)$ é uma função em $x$ que tem a forma de uma distribuição de probabilidade, e a integral em $x$ assume a forma de uma distribuição marginal da função $|\psi(x)|^{2}$. Seguindo este raciocínio, podemos imaginar uma distribuição de probabilidade $W(x, p)$ no espaço de posição e fase que contenha informações sobre o sistema nestas duas variáveis canônicas. Considerando tudo na integral (2.3.2), com exceção da integral em $x$, como uma distribuição de probabilidade, podemos escrever $|\tilde{\psi}(p)|^{2}$ como a distribuição marginal de $W(x, p)$

$$
|\tilde{\psi}(p)|^{2}=\int_{-\infty}^{\infty} d x W(x, p),
$$

tal que

$$
W(x, p) \equiv \int_{-\infty}^{\infty} \frac{d y}{2 \pi \hbar} \exp \left(-i \frac{p}{\hbar} y\right)\left\langle\psi(x+y / 2) \psi^{*}(x-y / 2)\right\rangle .
$$

O termo $\left\langle\psi(x+y / 2) \psi^{*}(x-y / 2)\right\rangle$ é conhecido como a matriz densidade do estado no espaço de posição. Mostra-se que a função probabilidade do estado do sistema no espaço de posição é dada por

$$
|\psi(x)|^{2}=\int_{-\infty}^{\infty} d p W(x, p) .
$$

Normalizando a função densidade $|\psi(x)|^{2}$, obtemos $\int_{-\infty}^{\infty} d x d p W(x, p)=1$, condição requerida para uma distribuição de probabilidade. Também é possível mostrar que $W(x, p)$ assume valo- 
res reais, mas que não são estritamente positivos. A possibilidade da função de Wigner assumir valores negativos é um indicativo do caráter quântico do sistema.

Com este formalismo, podemos obter o valor esperado de qualquer grandeza que dependa da posição e do momento $f(x, p)$, integrando-a sobre o espaço de fase mediada pela função de Wigner $\int_{-\infty}^{\infty} f(x, p) W(x, p)$.

Seria interessante agora discutir a função de Wigner para alguns estados do campo eletromagnético, como por exemplo estados coerentes e estados comprimidos.

\subsubsection{Estados coerentes}

O estado coerente é o estado de um oscilador harmônico que mais se aproxima de um oscilador clássico. Esta idéia é embasada no fato de que os valores esperados encontrados para os operadores de posição e momento são idênticos àqueles encontrados para as variáveis de posição e momento em um oscilador harmônico clássico. Portanto, um caso particular do estado coerente é o estado de vácuo, que é o estado fundamental do oscilador harmônico. Assim, lembrando que a função de onda para um oscilador harmônico no estado fundamental é dada por $\psi(x)=(a / \pi)^{1 / 4} \exp \left(-a x^{2} / 2\right)$, onde $a=m \omega / \hbar$, por substituição direta em (2.3.4) obtemos

$$
W(x, p)=\frac{1}{\pi \hbar} \exp \left[-\frac{m \omega}{\hbar}\left(\frac{p^{2}}{m^{2} \omega^{2}}+x^{2}\right)\right] .
$$

Como podemos ver, a função de Wigner para o estado de vácuo é uma gaussiana. O deslocamento da gaussiana no espaço de fase é caracterizado por um parâmetro conhecido como $\alpha$ e seu estado descrito por $|\alpha\rangle$. Mecanicamente falando, obtemos estes estados por um deslocamento de $\sqrt{2 / a} \Re(\alpha)$ da origem do oscilador harmônico, onde o termo que multiplica $\alpha$ tem a função de deixar o deslocamento com dimensão de posição. Este deslocamento deve ocorrer em uma escala de tempo menor que qualquer tempo envolvido no sistema, a fim de que a função de onda não se altere.

O deslocamento de uma função unidimensional em $x$, por exemplo $\psi(x)$, deve ser feito de tal maneira que o perfil da função deslocada $\tilde{\psi}(x)$ não seja alterado, isto é, $\tilde{\psi}(x)=\psi(x-\Delta x)$. Expandindo em série de Taylor temos $\tilde{\psi}(x)=\left(1-\Delta x \partial_{x}\right) \psi(x)=\exp \left(-\Delta x \partial_{x}\right) \psi(x)$. A derivada parcial em $x$ está relacionada com o operador de momento, que por sua vez pode ser escrito em termos dos operadores de criação e aniquilação da mecânica quântica, $\hat{a}^{\dagger}$ e $\hat{a}$ [10]. Assim temos que $\partial_{x}=\sqrt{a / 2}\left(\hat{a}-\hat{a}^{\dagger}\right)$, e escrevemos $-\Delta x \partial_{x}=-\Re(\alpha)\left(\hat{a}-\hat{a}^{\dagger}\right)$. Portanto podemos definir o operador deslocamento como

$$
\hat{D}(\alpha)=\exp \left(\alpha \hat{a}^{\dagger}-\alpha^{*} \hat{a}\right)
$$


Dessa forma, o estado coerente é entendido como um deslocamento do estado de vácuo.

$$
|\alpha\rangle=\hat{D}(\alpha)|0\rangle .
$$

A descrição do campo também pode ser feita através de suas quadraturas, semelhante à descrição do oscilador harmônico a partir das variáveis de posição e momento. Essas quadraturas são canonicamente conjugadas, implicando que suas variâncias estão sujeitas ao princípio da incerteza de Heisenberg, dando origem ao ruído quântico padrão.

O ruído quântico se origina da impossibilidade na mecânica quântica de se realizar medidas conjuntas de duas variáveis canonicamente conjugadas

$$
\operatorname{var}(X) \operatorname{var}(Y) \geq 1,
$$

devidamente normalizado.

A partir da (2.3.6), escrevemos a função de Wigner de um estado coerente em função das quadraturas de amplitude e fase, definindo as quadraturas segundo as relações equivalentes $X \equiv \sqrt{m \omega / \hbar} x, Y \equiv \sqrt{1 / \hbar m \omega} p$ e $\omega \equiv \sqrt{k / m}$

$$
W(X, Y)=\frac{1}{2 \pi} \exp \left[-\frac{1}{2}(X-\Re[\alpha])^{2}-\frac{1}{2}(Y-i \Im[\alpha])^{2}\right] .
$$

Para um campo coerente, o produto das variâncias das quadraturas satisfazem a igualdade na relação de incerteza de Heisenberg, e as incertezas nas quadraturas individuais são simétricas

$$
\operatorname{var}(X)=\operatorname{var}(Y)=1 \text {. }
$$

\subsubsection{Estados comprimidos}

O shot-noise descreve as flutuações de amplitude do estado coerente, e como vimos na subseção anterior, é um estado de menor incerteza. Certamente este não é o único estado que iguala o limite do princípio da incerteza. O estado coerente satisfaz o limite com pesos iguais nas flutuações das quadraturas, mas o princípio da incerteza de Heisenberg não requer que as variâncias sejam necessariamente iguais. De fato, $\operatorname{var}(X)$ ou $\operatorname{var}(Y)$ podem ser menores do que um, desde que o produto entre elas seja maior ou igual a um. Estados que se enquadram nesta categoria são conhecidos como estados comprimidos [42].

O estado comprimido pode ser descrito como uma compressão do estado gaussiano do oscilador harmônico no estado fundamental. A ideia é alterar, comprimindo, a concavidade do potencial harmônico que gerou o estado gaussiano. Este processo é não linear, e deve ser feito através de 
uma Hamiltoniana não linear nas equações de dinâmica.

Uma mudança suave sobre a frequência do oscilador, de $\omega$ para $\omega / s$, não irá alterar o estado fundamental do oscilador harmônico. Portanto, a função de onda que descreve este estado permanecerá a mesma, com exceção da substituição da frequência. Aplicando novamente a (2.3.4) para o estado fundamental do oscilador harmônico de frequência $\omega / s$, obtemos a função de Wigner para o estado comprimido

$$
W(x, p)=\frac{1}{\pi \hbar} \exp \left[-\frac{m \omega}{\hbar}\left(\frac{p^{2}}{s m^{2} \omega^{2}}+s x^{2}\right)\right]
$$

Podemos escrever também a função de Wigner em termos das quadraturas do campo

$$
W(X, Y)=\frac{1}{2 \pi} \exp \left[-\frac{s}{2}(X-\Re[\alpha])^{2}-\frac{1}{2 s}(Y-i \Im[\alpha])^{2}\right] .
$$

O cálculo das variâncias das quadraturas $X$ e $Y$ resultam em

$$
\operatorname{var}(X)=1 / s \quad ; \quad \operatorname{var}(Y)=s .
$$

Assim, para $s>1$, a variância na quadratura de amplitude é reduzida (compressão), enquanto que a variância na quadratura de fase é aumentada, mas o produto entre elas ainda satisfaz o limite mínimo do princípio de incerteza.

As linhas de contorno da função de Wigner são elipses e o tamanho dos semi-eixos maior e menor é dado pelo parâmetro s. É importante notar que na descrição acima do estado comprimido,
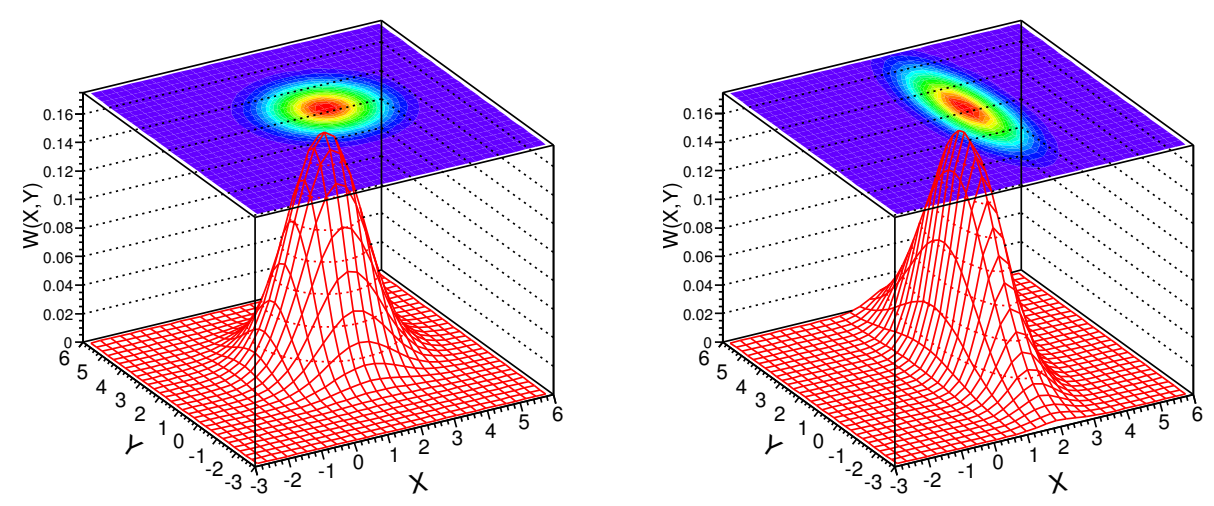

Figura 2.1: Figuras a) e b), são representações dos estados coerente e comprimido, respectivamente, pela função de Wigner. O valor usado para $\alpha$ foi $2+2 i$ e o parâmetro $s=3$. 
consideramos que o semi-eixo menor está alinhado com a quadratura $X$ e que o semi-eixo maior está alinhado com a quadratura $Y$. Essa configuração não é necessariamente verdade, a direção de máxima compressão pode fazer ângulos com as quadraturas. Esses casos são importantes no estudo da evolução temporal do estado comprimido [40].

Alterando o potencial harmônico, alteram-se também os operadores de criação e aniquilação do oscilador harmônico. No entanto, podemos escrever os novos operadores em termos dos antigos. Seja o potencial antigo descrito em termos de $\omega$, e o potencial comprimido descrito em termos de $\omega / s$. Então o operador comprimido $b$ é escrito como

$$
\hat{b}=\mu \hat{a}+\nu \hat{a}^{\dagger},
$$

onde $\mu=(\sqrt{1 / s}+\sqrt{s}) / 2$ e $\nu=(\sqrt{1 / s}-\sqrt{s}) / 2$. Nota-se imediatamente que os números complexos $\mu$ e $\nu$ obedecem a relação $|\mu|^{2}-|\nu|^{2}=1$. Transformações do tipo da (2.3.15), com a condição acima satisfeita, são conhecidas como transformações de Bogoliubov [24]. Estas transformações preservam o princípio da incerteza e os operadores comprimidos obedecem a mesma relação de comutação dos operadores do estado coerente

$$
\left[\hat{b}, \hat{b}^{\dagger}\right]=1
$$

Uma solução imediata para a equação (2.3.15) são as funções hiperbólicas. Não demonstrarei a solução geral das equação (2.3.15). Um argumento suficiente para a utilização das funções hiperbólicas é a relação que elas obedecem $\cosh ^{2}(r)-\sinh ^{2}(r)=1$. Portanto, escrevemos o operador $\hat{b}$ do estado comprimido em termos do operador $\hat{a}$ e $\hat{a}^{\dagger}$ do estado coerente como

$$
\hat{b}=\cosh (r) \hat{a}+\sinh (r) \hat{a}^{\dagger},
$$

o parâmetro $r$ é denominado de parâmetro de compressão.

Assim como definimos o operador de deslocamento para a produção de um estado coerente geral, podemos definir também o operador de compressão para geração do estado comprimido. O processo de geração de um estado comprimido, deve ser entendido como uma evolução temporal do estado coerente, quando este é submetido a uma Hamiltoniana adequada. Sendo um processo não linear, a Hamiltoniana mais simples que podemos imaginar, deve conter termos quadráticos em $\hat{a}$ e $\hat{a}^{\dagger}$

$$
\hat{H}=i \hbar \Omega\left(\zeta^{*} \hat{a}^{2}-\zeta \hat{a}^{\dagger 2}\right),
$$

onde $\zeta$ é a grandeza complexa que parametriza a compressão da variância a valores inferiores ao do estado coerente. Na representação de Heisenberg, com o auxílio da Hamiltoniana (2.3.18), 
podemos escrever as equações de dinâmica dos operadores

$$
\dot{\hat{a}}=-2 \Omega \zeta \hat{a}^{\dagger}
$$

e sua Hermitiana conjugada. Derivando temporalmente (2.3.19), e inserindo a equação de $\hat{a}^{\dagger}$, obtemos

$$
\ddot{\hat{a}}=4 \Omega^{2}|\zeta|^{2} \hat{a} .
$$

Diferentemente da dinâmica dos operadores para o oscilador harmônico, o termo proporcional ao operador $\hat{a}$ é estritamente positivo, levando a soluções do tipo $\hat{a} \cosh (\lambda t)$ e $\hat{a}^{\dagger} \sinh (\lambda t)$, onde $\lambda t=-2|\zeta| \Omega t$. Portanto, uma Hamiltoniana do tipo da (2.3.18), aplicada a um estado de vácuo leva a uma evolução temporal dos operadores $\hat{a}$ e $\hat{a}^{\dagger}$ na forma da expressão (2.3.17).

No formalismo de Heisenberg, o operador de compressão pode ser escrito como

$$
\hat{S}(\zeta)=\exp \left(\zeta^{*} \hat{a}^{2}-\zeta \hat{a}^{\dagger}\right)
$$

A função de Wigner (2.3.13), descreve um estado comprimido gerado inicialmente por uma compressão do vácuo seguido por um deslocamento $\alpha$. Em termos dos operadores, podemos escrever

$$
|c o m p\rangle=\hat{D}(\alpha) \hat{S}(s)|0\rangle .
$$

É importante registrar que é possível gerar o mesmo estado comprimido por um deslocamento inicial do vácuo seguido de uma compressão. No entanto, os parâmetros de deslocamento e compressão $\alpha$ e $s$ devem ser diferentes. De fato, temos

$$
|c o m p\rangle=S(s) D(\alpha)|0\rangle=D(\alpha / \sqrt{s}) S(s)|0\rangle .
$$

A luz convencionalmente gerada, através de um laser por exemplo, não possui propriedades quânticas do estado comprimido. Normalmente a luz de um laser pode ser aproximada por um estado coerente. O processo de geração do estado comprimido requer uma Hamiltoniana não linear do tipo (2.3.18). Como veremos, processos que envolvem o efeito Kerr, com base na mistura de quatro ondas $4 \mathrm{WM}$, são capazes de gerar compressão nas quadraturas.

\subsection{Medida da função de Wigner}

A teoria de medição da função de Wigner é uma área de estudo conhecida como tomografia quântica, cujo nome é baseado na semelhança entre o processo de medida do estado quântico 
descrito pela função de Wigner, e o método de mapeamento por divisão em fatias de um corpo, através de ondas de raios X, por exemplo. A ideia principal é transferir para o domínio quântico o algoritmo de construção de imagens em duas dimensões de corpos densos a partir de projeções unidimensionais em diferentes direções.

Vimos que na representação dos estados quânticos pela função de Wigner, obtemos informações a respeito da distribuição de probabilidade do estado na variável de posição a partir da distribuição marginal da função de Wigner no espaço de momento

$$
\rho(x)=\int_{-\infty}^{\infty} d p W(x, p) .
$$

Graficamente, podemos entender a integração na variável de momento como uma projeção na quadratura de posição da função de Wigner, que implica na projeção de toda a informação do estado sobre a variável de posição. Obviamente perdemos informação se nos limitarmos apenas a essa medida. Uma alternativa possível, seria projetar a informação na variável de momento, integrando a função de Wigner em $x$. Generalizando, podemos projetar a função de Wigner numa superposição de posição e momento em uma direção arbitrária $\theta$. Um procedimento

a)

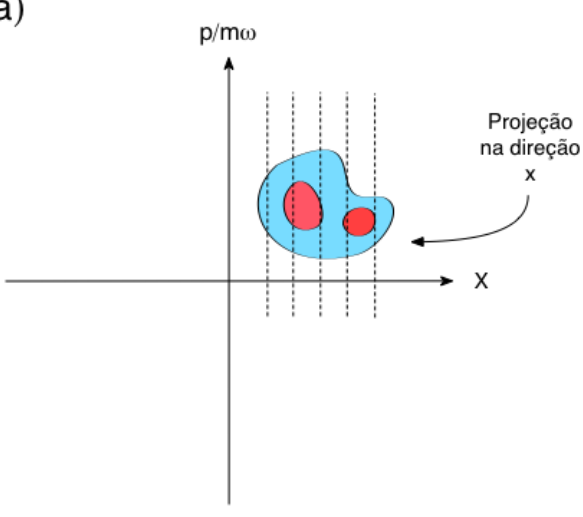

b)

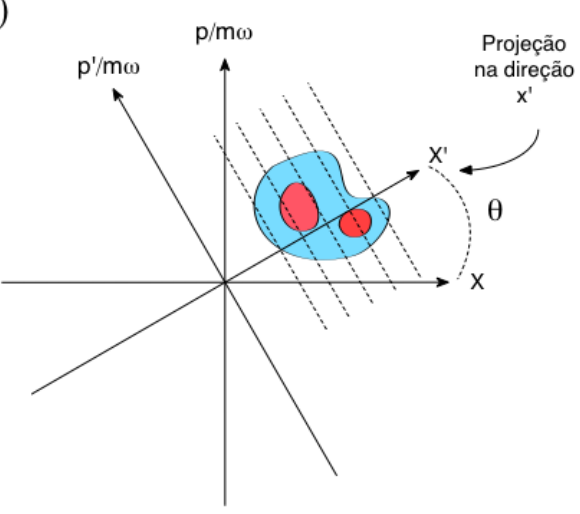

Figura 2.2: Tomografia do estado quântico. Na figura a) medimos a projeção de um estado quântico qualquer no espaço de posição. A figura b) mostra graficamente a projeção do estado em uma quadratura generalizada, deslocada angularmente de $\theta$ do eixo de posição.

equivalente, é realizar uma rotação randômica de $\theta$ do espaço de fase e projetar a função de Wigner na nova variável de posição $x^{\prime}$

$$
\rho_{\theta}\left(x^{\prime}\right)=\int_{-\infty}^{\infty} d p^{\prime} W\left(x^{\prime}, p^{\prime}\right) .
$$


Obtemos mais informações a respeito do estado conforme realizamos medidas em direções diferentes, e é possível reconstruir inteiramente o estado quântico por meio de uma rotação contínua do espaço de fase.

O ponto de partida para se medir a função de Wigner é definir o operador de rotação de quadratura

$$
\hat{X}_{\theta}=\cos \theta \kappa \hat{x}+\sin \theta \frac{\hat{p}}{\hbar \kappa},
$$

onde $\hat{x}$ e $\hat{p}$ são os operadores posição e momento, respectivamente. O parâmetro $\kappa$ é somente um fator de escala.

Assumimos que a todo operador $\hat{X}_{\theta}$, está associado um auto-vetor $\left|X_{\theta}\right\rangle$, com o respectivo auto-valor $X_{\theta}$, e que este conjunto de operadores, diferenciados pelo parâmetro $\theta$, satisfaçam a resolução da unidade $\int d X_{\theta}\left|X_{\theta}\right\rangle\left\langle X_{\theta}\right|=\hat{1}$. Devemos agora derivar a função de Wigner $W_{\left|X_{\theta}\right\rangle}$ para o operador de rotação de quadratura, e para isso é necessário projetar os auto-estados sobre o espaço de posição

$$
\left\langle x\left|\hat{X}_{\theta}\right| X_{\theta}\right\rangle=X_{\theta}\left\langle x \mid X_{\theta}\right\rangle
$$

Utilizando a relação (2.4.3), obtemos a função de onda no espaço de posição do estado de rotação de quadratura

$$
\psi\left(x ; X_{\theta}\right)=N(\theta) \exp \left[-i \frac{\left(\cos \theta \kappa x-X_{\theta}\right)^{2}}{2 \sin \theta \cos \theta}\right] .
$$

Em princípio, o fator de normalização $N(\theta)$ pode depender do ângulo de rotação $\theta$ e do autovalor $X_{\theta}$, mas devido à função de onda $\psi\left(x ; X_{\theta}\right)$ ter o formato de uma Gaussiana apenas com parte imaginária, esta não é normalizável da maneira convencional, devendo fazê-la em termos da função delta de Dirac, e o processo é simplificado se considerarmos que o fator de normalização dependa somente de $\theta$, cujo valor encontrado é $|N(\theta)|^{2}=\kappa / 2 \pi \sin \theta$.

Calculamos a função de Wigner para o auto-estado $\left|X_{\theta}\right\rangle$, inserindo diretamente a função de onda (2.4.5) na (2.3.4)

$$
W_{\left|X_{\theta}\right\rangle}=\frac{1}{2 \pi \hbar} \delta\left[X_{\theta}-\left(\cos \theta \kappa x+\sin \theta \frac{p}{\hbar \kappa}\right)\right] .
$$

Portanto, a função de Wigner para o estado de rotação de quadratura é uma delta de Dirac ao longo da linha $\cos \theta \kappa x+\sin \theta p / \hbar \kappa$, e exerce papel fundamental na reconstrução completa do estado quântico, pois ela permite medir a distribuição de probabilidade do estado, ao longo de uma linha específica representada pela direção $\theta$. Portanto, é possível reconstruir o estado quântico $\hat{\rho}$ através da medida

$$
W\left(X_{\theta}\right)=\operatorname{Tr}\left\{\left|X_{\theta}\right\rangle\left\langle X_{\theta}\right| \hat{\rho}\right\},
$$


para vários ângulos $\theta^{4}$. A função (2.4.7) é denominada de distribuição de quadratura. Com o auxílio da (2.4.6) obtemos

$$
W\left(X_{\theta}\right)=\int_{-\infty}^{\infty} d x \int_{-\infty}^{\infty} d p \delta\left[X_{\theta}-\left(\cos \theta \kappa x+\sin \theta \frac{p}{\hbar \kappa}\right)\right] W_{\hat{\rho}}(x, p) .
$$

A integral (2.4.8) possibilita uma interpretação bem interessante. A medida da densidade de quadratura é feita pela interseção da função de Wigner do estado quântico que se deseja medir com a função $\delta$, ou seja, a distribuição de quadratura (2.4.7), são "fatias"da função de Wigner do estado quântico. Portanto, podemos medir o estado quântico completo do sistema a partir de várias medidas da função $W\left(X_{\theta}\right)$.

A partir das medidas realizadas, é possível reconstruir o estado do sistema invertendo a equação (2.4.8). Para inverter esta equação, é necessário escrever a função delta como uma transformada de Fourier, e seguir as etapas necessárias para encontrar a transformada de Fourier inversa [40]

$W_{\hat{\rho}}(x, p)=\frac{1}{4 \pi^{2} \hbar} \int_{-\infty}^{\infty} d t|t| \int_{-\pi / 2}^{\pi / 2} d \theta \int_{-\infty}^{\infty} d X_{\theta} \exp \left[i t\left(X_{\theta}-\kappa x \cos \theta-\frac{p}{\hbar \kappa} \sin \theta\right)\right] W\left(X_{\theta}\right)$.

As técnicas de medida das quadraturas, assim como a rotação do espaço de fase para a reconstrução da função de Wigner, podem ser encontradas em técnicas conhecidas por homodinagem.

\subsection{Medidas das variâncias}

Na seção anterior, vimos como podemos reconstruir a função de Wigner de um sistema quântico. Muitas vezes não estamos interessados em reconstruir o estado por completo, sendo suficiente investigar apenas algumas propriedades do mesmo. No caso de medidas realizadas sobre o campo eletromagnético, frequentemente estamos interessados nas medidas das variâncias das quadraturas, que sob algumas considerações, como admitir uma distribuição gaussiana do estado, pode representar um perfil bidimensional da distribuição de Wigner. Existem diferentes técnicas para se medir as variâncias das quadraturas, onde as mais conhecidas, são a detecção homodina [57, 41] e a deteç̧ão por cavidades [25] , também conhecida como rotação da elipse de ruído [53].

A detecção homodina se destaca por sua grande utilização em muitos trabalhos envolvendo medidas de ruído. Entretanto, sua aplicação de uma forma eficiente impõe certas condições que às vezes podem não ser satisfeitas. A limitação de sua utilização se deve às características do

\footnotetext{
${ }^{4}$ Mostra-se que é necessário variar o ângulo $\theta$ de $-\pi / 2$ até $\pi / 2$.
} 
campo de referência, denominado de oscilador local, que devem se assemelhar às características do campo a ser medido. Estas características se referem à frequência, fase, perfil espectral e modo espacial, além de que, a intensidade do oscilador local deve ser bem maior do que a intensidade do campo de interesse [34].

Por causa da utilização exclusiva da técnica de rotação de elipse neste trabalho, em razão de suas vantagens com relação às limitações da detecção homodina, irei descrever de uma maneira resumida sua implementação como forma de medida de ruído.

O fotodetector é constituído basicamente de um fotodiodo ${ }^{5}$ e um circuito eletrônico de amplificação constituído por amplificadores operacionais. O funcionamento do fotodiodo é baseado no princípio do efeito fotoelétrico, explicado por Einstein. O fóton, considerado como um pacote discreto de energia definida, interage com um objeto metálico podendo liberar um elétron, e a energia cinética do elétron liberado varia linearmente com a energia do fóton. A capacidade do sistema de deteç̧ão de distinguir fótons individuais e produzir respostas por meio de correntes clássicas é conhecida como eficiência quântica, que leva em conta ainda a taxa de perda de fótons na detecção. Portanto, para uma deteç̧ão com eficiência quântica unitária, o aparato consegue distinguir cada fóton individualmente, e com isso temos, além da informação do número médio de fótons que chegam ao detector, a informação sobre as flutuações estatísticas dos fótons decodificadas em termos de flutuações na corrente.

Desta forma, o fotodetector consegue extrair informações sobre a intensidade do campo, iluminando diretamente o fotodiodo com a onda eletromagnética. No entanto, o mesmo não acontece com a fase. O fotodiodo não consegue determinar um valor de referência para a fase de uma onda eletromagnética, nem suas flutuações em torno do valor médio. Para extrair alguma informação sobre a fase do campo, devemos compará-la com a fase de um campo de referência conhecido, através de um experimento interferométrico, como por exemplo o Mach-Zehnder.

Dada uma onda eletromagnética monocromática e com a fase bem definida, podendo ser descrita por uma senóide por exemplo, suponha que possamos dividir este feixe em 50\%, por um divisor de feixes 50/50, e conduzi-los por dois braços idênticos, mesmo comprimento, e recombiná-los novamente por um divisor de feixes idênticos ao primeiro. Neste caso, ambos os campos divididos alcançarão o divisor de feixe com a mesma fase e se recombinarão resultando em um campo idêntico ao incidente. Agora, alterando levemente o tamanho de um dos braços, as fases dos campos que chegam ao segundo divisor serão levemente diferentes, e um padrão de interferência poderá ser observado dependendo da diferença de fase. Caso a diferença de trajetória resulte em uma diferença de fase de $\pi$, uma interferência destrutiva irá acontecer e a intensidade do feixe recombinado será nula. Portanto, variando a fase relativa entre as ondas eletromagnéticas, re-

\footnotetext{
${ }^{5}$ Existem atualmente fotodiodos confeccionados com materiais semicondutores ou metálicos. O menor tempo de resposta deste último com relação ao fotodiodo de junção PN é a principal diferença dos dois modelos.
} 
flete numa variação de intensidade do campo após um processo interferométrico, possibilitando adquirir informação sobre o valor médio e as flutuações de fase pelo fotodiodo.

Esse é o princípio de funcionamento de qualquer técnica para se extrair informação da fase do campo. A detecção homodina nada mais é do que um interferômetro de Mach Zehnder, no qual, por um dos braços, transmitimos o campo a ser estudado, e pelo outro braço, o campo de referência é responsável por variar a fase relativa. Normalmente estamos interessados apenas nas flutuações de fase do campo de interesse. Portanto, a interferência que ocorre na recombinação dos feixes deve ser suficiente apenas para detectar as variações na intensidade devido às flutuações de fase, mantendo o seu valor médio pouco alterado. A importancia disto reside no fato de que o fotodiodo funciona eficientemente quando trabalha em uma faixa de intensidade adequada, possuindo uma intensidade razoável para se distinguir da luz de fundo, mas que também fique abaixo do limite de saturação do fotodiodo.

A técnica de rotação de elipse faz uso de cavidades ópticas para alterar a fase do campo de referência com o campo de interesse. Neste caso, tomamos como campo de referência a própria portadora, que corresponde a uma região do espectro com um número de fótons significativos. O deslocamento de fase é dado com relação às bandas laterais, que são as regiões espectrais que compõem o sinal temporal do campo eletromagnético, mas que possuem um número reduzido de fótons, quase zero. O fato de ser desnecessário a presença de um campo de referência, intenso, separado do campo a ser medido, torna a detecção com cavidades bastante atraente. Uma cavidade óptica do tipo Fabry-Perot nada mais é do que uma caixa fechada internamente refletora, que sob condições de contorno adequadas, limitamos a existência de apenas alguns modos discretos. A figura 2.3 mostra a estrutura principal de uma cavidade de análise. O acoplamento de entrada é feito por um espelho parcialmente refletor mas com baixa transmissão. O segundo espelho não precisa ter necessariamente reflexão unitária, que além do mais, por limitações técnicas, dificilmente encontra-se um espelho com $R=1$. Devemos considerar, nestes casos, que o campo intracavidade estará sofrendo influência do ruído de vácuo, e portanto adicionando ruído ao campo de interesse. Discutiremos mais sobre a influência do vácuo sobre uma cavidade nos capítulos 3 e 4 . Considerando os índices de reflexão definidos por $r_{\imath}^{2}=R_{\imath}$, com $\imath=1$ ou 2 , e também os índices de transmissão $t_{\imath}^{2}=T_{\imath}$, com 1 para o espelho de entrada e 2 para o segundo espelho, obtemos os principais parâmetros que descreve a cavidade: a frequência de ressonância $\nu_{c}$, a distância entre duas frequências de ressonância $F S R=c / L$, a finesse

$$
F=\frac{\pi\left(R_{1} R_{2}\right)^{1 / 4}}{1-\sqrt{R_{1} R_{2}}}
$$

e a largura de linha $\delta \nu_{c}=F S R / F$, onde $L$ é o comprimento da cavidade [3]. A finesse é um parâmetro da cavidade associado com o fator de qualidade da mesma, e fornece a idéia de 


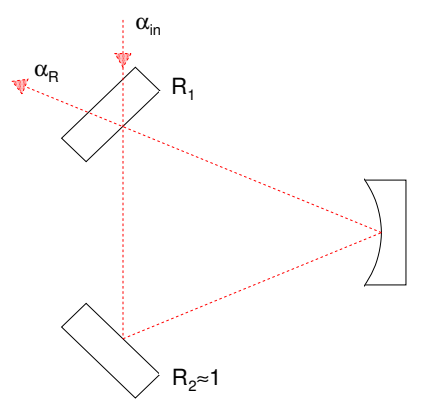

Figura 2.3: Cavidade óptica simbolizando a cavidade de análise das quadraturas dos campos. Os espelhos que constituem a cavidade devem possuir alto índice de reflexão. A amplitude do campo refletido $\alpha_{R}$ é a soma da amplitude do campo incidente $\alpha_{i n}$ com o vácuo, que pode entrar pelo segundo espelho.

quanto a cavidade consegue armazenar luz em seu interior.

O modo do campo pode ser escrito como (2.1.2)

$$
\alpha(t)=\bar{\alpha}+\delta \alpha(t)
$$

onde $\bar{\alpha}$ representa o valor médio do campo e $\delta \alpha(t)$ sua flutuação. Por conseguinte, o campo incidente sobre a cavidade $\alpha_{i n}(t)=\bar{\alpha}_{i n}+\delta \bar{\alpha}_{i n}(t)$ resulta no campo refletido $\alpha_{R}(t)=\bar{\alpha}_{R}+\delta \bar{\alpha}_{R}(t)$ sob a influência das flutuações de vácuo com média nula $\alpha_{v}(t)=\delta \alpha_{v}(t)$. Definindo a frequência de análise em unidades de largura de linha da cavidade, $\nu^{\prime}=\nu / \delta \nu_{c}$, temos a seguinte relação para o modo do campo nesta frequência [19]

$$
\alpha_{R}\left(\nu^{\prime}\right)=r\left(\Delta+\nu^{\prime}\right) \alpha_{i n}\left(\nu^{\prime}\right)+t\left(\Delta+\nu^{\prime}\right) \alpha_{v}\left(\nu^{\prime}\right)
$$

com os coeficientes de reflexão e transmissão dados por

$$
r(\Delta)=\frac{-r_{1}+r_{2} \exp (i 2 \pi \Delta / F)}{1-r_{1} r_{2} \exp (i 2 \pi \Delta / F)} \text { e } t(\Delta)=\frac{t_{1} t_{2} \exp (i \pi \Delta / F)}{1-r_{1} r_{2} \exp (i 2 \pi \Delta / F)}
$$

onde $\Delta=\left(\nu_{0}-\nu_{c}\right) / \delta \nu_{c}$ é a dessintonia da frequência da portadora com relação à frequência de ressonância da cavidade em unidades de largura de linha da cavidade.

A curva da figura 2.4 mostra o módulo ao quadrado e a fase do coeficiente de reflexão $r(\Delta)$ em função da dessintonia entre a frequência da portadora e a ressonância da cavidade. A portadora ao passar pela região ressonante da cavidade de análise, sofre uma depleção na intensidade e recebe uma fase de $2 \pi$ com relação às bandas laterais que estão longe da ressonância. A 


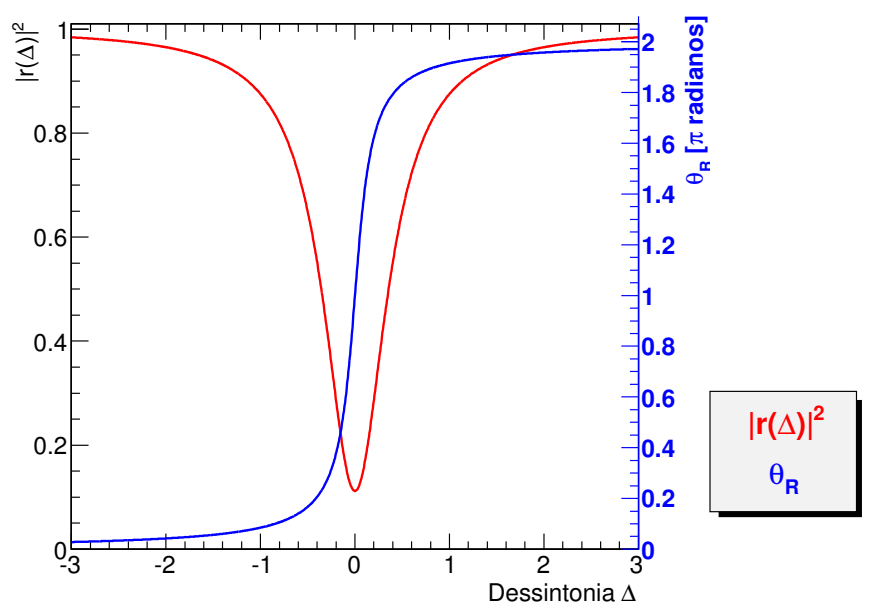

Figura 2.4: Gráfico do módulo ao quadrado $|r(\Delta)|^{2}$ e a fase $\theta_{r}(\Delta)$ do coeficiente de reflexão em função da dessintonia $\Delta$. Os valores dos parâmetros utilizados foram: $R_{1}=0.99, R_{2}=0.995 \mathrm{e}$ $F=337$.

consequência deste deslocamento de fase é a possibilidade de se medir as flutuações de fase das bandas laterais.

A flutuação normalizada da quadratura de amplitude do campo é obtida a partir da diferencial do módulo ao quadrado da expressão (2.5.2), dividida pelo módulo do campo [53]

$$
\delta p(v)=\exp (-i \phi) \delta \alpha(\nu)+\exp (i \phi) \delta \alpha^{*}(-\nu)
$$

A quadratura de fase é a variável canônica em quadratura

$$
\delta q(v)=-i\left[\exp (-i \phi) \delta \alpha(\nu)-\exp (i \phi) \delta \alpha^{*}(-\nu)\right]
$$

O campo $\alpha(t)$, ao ser acoplado com a cavidade de análise sofrerá a influência desta ao entrar em ressonância. Esta influência é entendida como um acréscimo de fase na flutuação da quadratura de amplitude da banda lateral, ou da portadora, correspondente à frequência de ressonância da cavidade, como podemos ver na figura 2.5 .

Quando a cavidade de análise começa a entrar em ressonância com a banda lateral negativa (-), a fase aplicada é zero e medimos o ruído de amplitude. Conforme a banda lateral negativa vai entrando em ressonância, a fase aplicada pela cavidade vai aumentando. Quando a fase atinge o valor de $\pi$, a cavidade mede o ruído de fase. Neste ponto, a banda lateral começa a sair de ressonância e a fase continua a aumentar. $\operatorname{Em} \theta_{R}=2 \pi$, a cavidade mede novamente o ruído de amplitude. Depois deste ponto, a cavidade de análise começa a entrar em ressonância com 


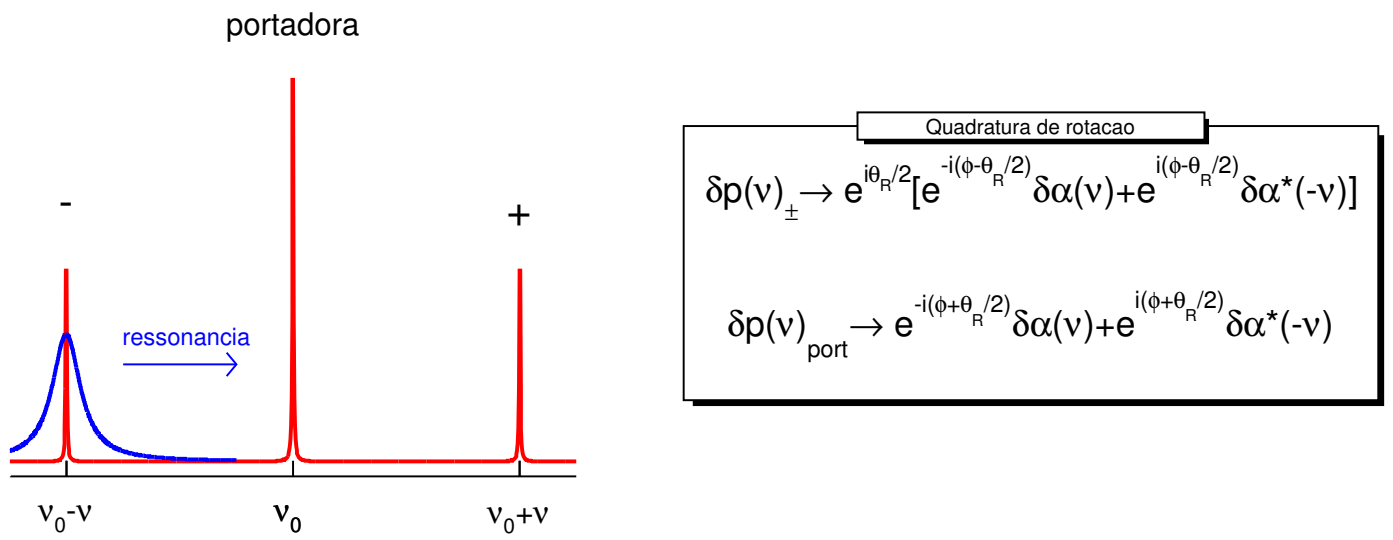

Figura 2.5: Representação da portadora e das bandas laterais. O efeito da cavidade de análise sobre o campo complexo $\alpha(t)$ é adicionar uma fase de $\theta_{R}$ sobre a quadratura de amplitude da portadora e das bandas laterais.

a portadora. Longe da ressonância, ela mede o ruído de amplitude da portadora. O ruído de fase da portadora é medido em $\theta_{R}=\pi / 2$ e $3 \pi / 2$. Para a portadora, em $\theta_{R}=\pi$, longe da ressonância tanto no espectro negativo quanto no positivo, medimos o ruído de amplitude. Um processo semelhante ao que ocorre com a banda lateral negativa, ocorre com a banda lateral positiva $(+)$.

Podemos relacionar a quadratura de amplitude do campo refletido com as quadraturas de amplitude e fase do campo de entrada juntamente com as flutuações de vácuo [53]

$$
\delta p_{R}\left(\Delta, \nu^{\prime}\right)=g_{p} \delta p_{i n}\left(\nu^{\prime}\right)+i g_{q} \delta q_{i n}\left(\nu^{\prime}\right)+g_{v p} \delta v_{p}\left(\nu^{\prime}\right)+i g_{v q} \delta v_{q}\left(\nu^{\prime}\right)
$$

onde $\delta v=\exp (i \phi)\left(\delta v_{p}+i \delta v_{q}\right) / 2$ são as flutuações do vácuo, e

$$
\begin{aligned}
& 2 g_{p}=\exp \left[-i \theta_{R}(\Delta)\right] r\left(\Delta+\nu^{\prime}\right)+\exp \left[i \theta_{R}(\Delta)\right] r^{*}\left(\Delta-\nu^{\prime}\right), \\
& 2 g_{q}=\exp \left[-i \theta_{R}(\Delta)\right] r\left(\Delta+\nu^{\prime}\right)-\exp \left[i \theta_{R}(\Delta)\right] r^{*}\left(\Delta-\nu^{\prime}\right), \\
& 2 g_{v p}=\exp \left[-i \theta_{R}(\Delta)\right] t\left(\Delta+\nu^{\prime}\right)+\exp \left[i \theta_{R}(\Delta)\right] t^{*}\left(\Delta-\nu^{\prime}\right), \\
& 2 g_{v q}=\exp \left[-i \theta_{R}(\Delta)\right] t\left(\Delta+\nu^{\prime}\right)-\exp \left[i \theta_{R}(\Delta)\right] t^{*}\left(\Delta-\nu^{\prime}\right) .
\end{aligned}
$$

O módulo dos coeficientes acima $(2.5 .8$ - 2.5.11), representam os pesos das quadraturas conjugadas do campo de entrada, amplitude e fase, que compõem a quadratura de amplitude do 
campo refletido. A partir da expressão (2.5.7), podemos ver explicitamente a atuação da cavidade sobre as flutuações das quadraturas do campo de entrada, em função da dessintonia. Considerando apenas o módulo dos coeficientes que acompanham as flutuações, a figura 2.6 mostra que para dessintonias grandes $(\Delta>8)$, a cavidade de análise não exerce influência sobre o campo, e apenas o ruído na quadratura de amplitude é medido. A máxima conversão entre ruído de amplitude para ruído de fase da banda lateral negativa acontece para valor de dessintonia próximo da frequência de análise $\left(\Delta \approx-\nu^{\prime}\right)$. Para dessintonias menores, medimos uma mistura entre ruídos de amplitude e fase, e a influência de um ruído sobre o outro depende da frequência de análise. Para dessintonias pequenas, a cavidade de análise começa a entrar em ressonância com a portadora. Em $\Delta \approx 1 / 2$, medimos o ruído de fase para a portadora, e em $\Delta=0$, o ruído de amplitude. No lado positivo do espectro, medimos também o ruído de fase da banda lateral positiva na dessintonia próxima da frequência de análise.

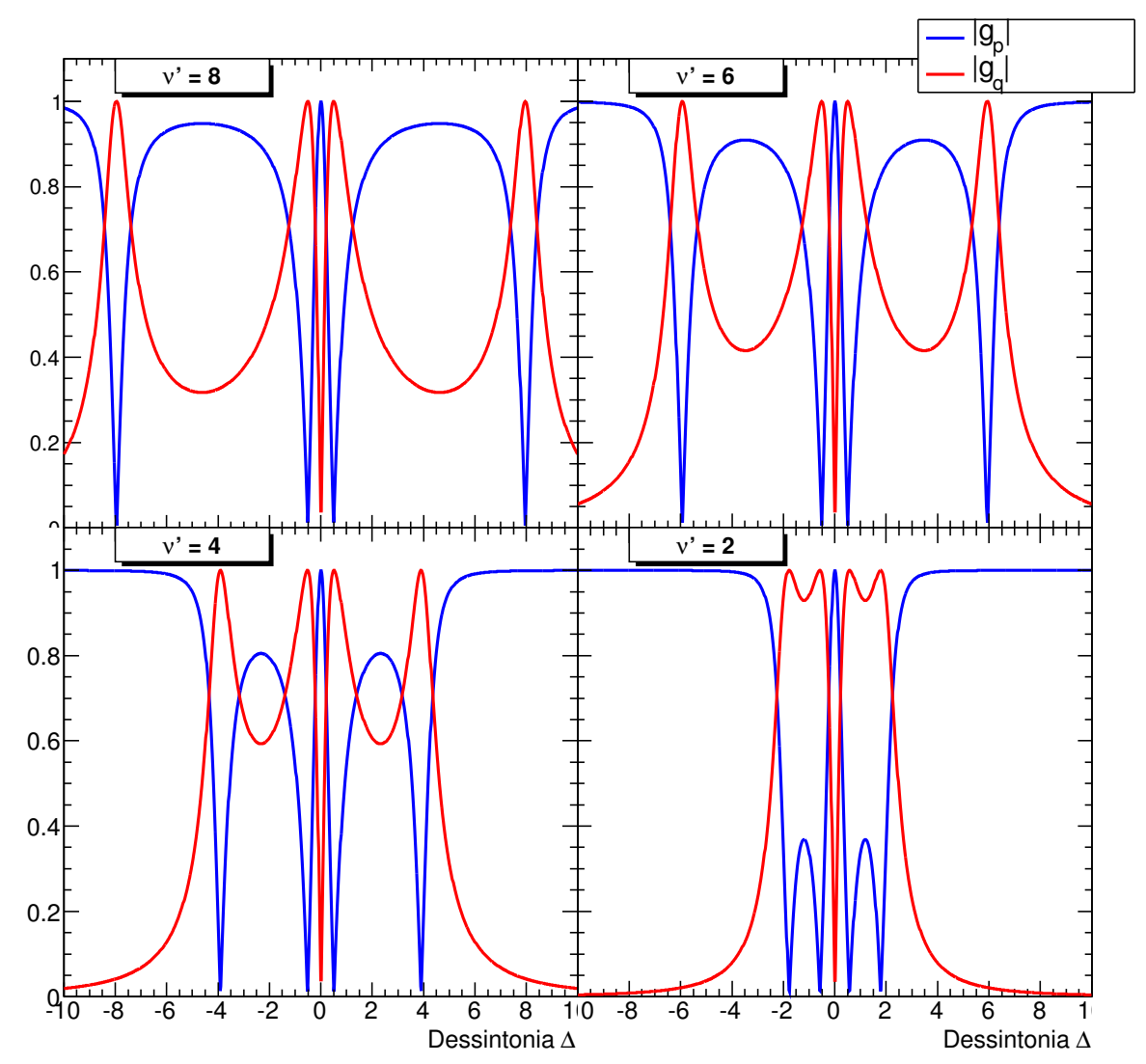

Figura 2.6: Curvas dos módulos $\left|g_{p}\right|$ e $\left|g_{q}\right|$ em função da dessintonia da cavidade para diferentes valores de $\nu^{\prime}$. A cavidade foi considerada bem fechada $\left(R_{2}=1 ; R_{1} \rightarrow 1\right)$, e o efeito das flutuações de vácuo desprezada.

É importante notar que, quando trabalhamos com frequências de análise pequenas, os ruídos 
de fase das bandas laterais se aproximam do ruído de fase da portadora, tornando-se quase indistinguíveis em $\nu^{\prime}=2$. Esse fato limita inferiormente a frequência que queremos investigar. De fato, mostra-se que, para uma completa separação dos ruídos das bandas laterais com a portadora, devemos analisar o espectro em regiões tal que $\nu^{\prime}>\sqrt{2}$.

\subsection{Calibração de shot-noise}

A calibração do shot-noise é um procedimento no qual se compara o ruído de amplitude de um estado qualquer $|a\rangle$, com o ruído de vácuo. Uma maneira simples de se fazer isso é inserir esse estado através de uma porta de um beam-splitter 50/50(BS), e pela outra porta inserir um estado coerente ${ }^{6}$. Com os dois estados divididos igualmente na saída do BS, medimos a variância da soma e subtração de suas intensidades. Se o BS possuir índices de transmissão e

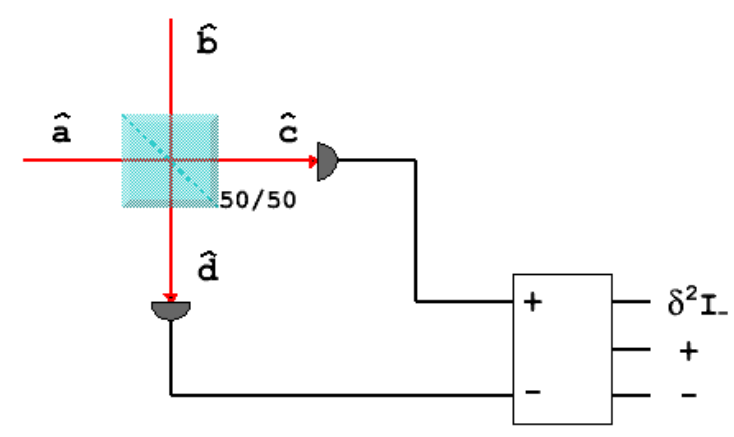

Figura 2.7: Diagrama representando a medida do shot-noise

reflexão $t$ e $r$, pela conservação de energia obtemos $|r|^{2}+|t|^{2}=1$ e $r t^{*}+t r^{*}=0$. A consequência da transformação de beam-splitter é o surgimento de uma fase de $\pi$ em um dos campos refletidos [3].

Na detecção balanceada, $|t|=|r|=1 / \sqrt{2}$, e obtemos para os estados de saída $|c\rangle$ e $|d\rangle$

$$
\begin{aligned}
& |c\rangle=\frac{1}{\sqrt{2}}(|a\rangle+|b\rangle), \\
& |d\rangle=\frac{1}{\sqrt{2}}(|a\rangle-|b\rangle) .
\end{aligned}
$$

\footnotetext{
${ }^{6}$ Não há uma exigência física para que o estado seja coerente, é feita essa suposição para facilitar a análise.
} 
Se o estado de entrada $|a\rangle$ é um estado de Fock de um laser por exemplo, os detectores medem a corrente fotoelétrica devido à interação do fóton com o elétron do fotodiodo. Ou seja, os fotodetectores medem o número de fótons - intensidade $-k c^{\dagger} c$ e $k d^{\dagger} d$, onde $k$ é um termo de amplitude proveniente da eletrônica do sistema de detecção.

A soma e a diferença das intensidades são dadas por

$$
\begin{aligned}
& I_{+}=k\left(\hat{a}^{\dagger} \hat{a}+\hat{b}^{\dagger} \hat{b}\right), \\
& I_{-}=k\left(\hat{a}^{\dagger} \hat{b}+\hat{a} \hat{b}^{\dagger}\right) .
\end{aligned}
$$

Como estamos interessados em medir o ruído quântico do estado $|a\rangle$, utilizando um estado de referência $|b\rangle$, não desejamos que estes dois estados possuam algum tipo de correlação. Portanto, vamos assumir experimentalmente como independentes, ou seja, que possam ser escritos em dois espaços de Hilbert distintos $|a\rangle \otimes|\beta\rangle$, onde $|\beta\rangle$ é um estado coerente

$$
\hat{b}|\beta\rangle=\beta|\beta\rangle .
$$

Podemos então calcular agora as variâncias da soma e da subtração das intensidades

$$
\operatorname{var}\left(I_{ \pm}\right)=\left\langle I_{ \pm}^{2}\right\rangle-\left\langle I_{ \pm}\right\rangle^{2}
$$

Com o uso da (2.6.3) os cálculos das variâncias saem diretamente

$$
\begin{gathered}
\operatorname{var}\left(I_{-}\right)=k^{2}\left\langle\hat{a}^{\dagger} \hat{a}\right\rangle+k^{2}\left[\left\langle\left(\beta \hat{a}^{\dagger}+\beta^{*} \hat{a}\right)^{2}\right\rangle-\left(\beta\left\langle\hat{a}^{\dagger}\right\rangle+\beta^{*}\langle\hat{a}\rangle\right)^{2}\right], \\
\operatorname{var}\left(I_{+}\right)=k^{2}\left[\left\langle: \operatorname{var}\left(n_{a}\right):\right\rangle+\left\langle\hat{a}^{\dagger} \hat{a}\right\rangle+|\beta|^{2}\right] .
\end{gathered}
$$

Onde $\left\langle: \operatorname{var}\left(n_{a}\right):\right\rangle$ é a variância do operador número do campo $|a\rangle$ em ordem normal ${ }^{7}$.

O autovalor do operador $\hat{b}$ é uma grandeza complexa, e pode ser escrita como $\beta=|\beta| e^{i \theta}$. A quadratura generalizada do campo eletromagnético pode ser expressa em termos dos operadores como $X_{\phi}=\hat{a}^{\dagger} e^{i \phi}+\hat{a} e^{-i \phi}$. Reescrevendo as variâncias da soma e subtração das intensidades em termos destas grandezas

$$
\begin{gathered}
\operatorname{var}\left(I_{-}\right)=k\left\langle I_{a}\right\rangle+k\left\langle I_{b}\right\rangle \operatorname{var}\left(X_{\phi}^{(a)}\right), \\
\operatorname{var}\left(I_{+}\right)=k^{2}\left\langle: \operatorname{var}\left(n_{a}\right):\right\rangle+k\left\langle I_{a}\right\rangle+k\left\langle I_{b}\right\rangle .
\end{gathered}
$$

\footnotetext{
${ }^{7} \mathrm{~A}$ ordem normal é aquela na qual os operadores de construção aparecem na frente dos operadores de aniquilação. A saber, a variância do operador número do campo $\hat{a}$ em ordem normal é definida como $\left\langle a^{\dagger} a^{\dagger} a a\right\rangle-\left\langle a^{\dagger} a\right\rangle^{2}$.
} 
Uma situação particularmente interessante ocorre quando tratamos o campo $|\beta\rangle$ como um modo de vácuo, ou seja, $|\beta\rangle=|0\rangle$. Quando isso ocorre, as variâncias são dadas por:

$$
\begin{gathered}
\operatorname{var}\left(I_{-}\right)=k\left\langle I_{a}\right\rangle, \\
\operatorname{var}\left(I_{+}\right)=k^{2}\left\langle: \operatorname{var}\left(n_{a}\right):\right\rangle+k\left\langle I_{a}\right\rangle .
\end{gathered}
$$

Note que a variância de $I_{-}$é proporcional à intensidade $I_{a}$. A verificacão deste fato serve para testar a qualidade do sistema de detecção em eliminar o ruído clássico do campo. Para garantirmos que o campo seja coerente, devemos verificar que (2.6.9) e (2.6.10) são obedecidas para todas as quadraturas, e isso é feito com o auxílio de um oscilador local em $|b\rangle$. Verificou-se

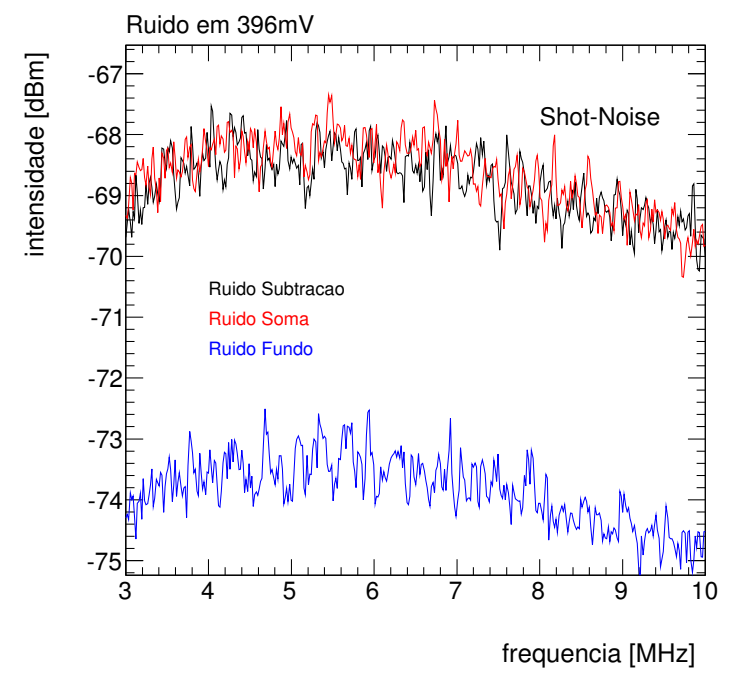

Figura 2.8: Espectro de ruído do Laser de subtração, soma e ruído de fundo, indo de $3 \mathrm{MHz}$ à $10 \mathrm{MHz}$ em unidades de $\mathrm{dBm}$.

experimentalmente que a detecção balanceada que empregamos pode de fato eliminar o ruído clássico do laser de tal modo que na subtração dos campos mede-se o ruído quântico padrão. Após a divisão do campo em $50 \%$ pelo $B S$, os campos foram focalizados nos detectores. Apesar dos fotodiodos ETX 300, produzidos pela EPITAXX, nos permitirem realizar medições em até $300 \mathrm{MHz}$, a eletrônica de amplificação deixa de ter uma boa resposta acima de $60 \mathrm{MHz}$. Portanto em todas as medidas de ruído procuramos ficar bem abaixo deste limite. As saídas dos fotodiodos passam por um eletrônica cujo sinal DC é separado e monitorado pelo osciloscópio. Cada sinal AC é amplificado independentemente e em seguida misturado pela eletrônica para realizar a subtração. O ruído de fundo, que inclui o ruído eletrônico e as flutuações térmicas, é medido bloqueando os campos antes dos detectores e descontado na medida do ruído total. 
A medida foi efetuada com o analisador de espectro da Agilent, modelo E4401B. O analisador estava configurado para medir em tempo real uma largura de banda de $100 \mathrm{KHz}$, e a resolução de video ajustada para $1 \mathrm{KHz}$. As medidas foram tomadas em uma média de 10 segundos.

A calibração de shot-noise consiste em comparar o ruído quântico do laser com as flutuações de vácuo, excitadas na outra porta do BS. Segundo a relação (2.6.9), devemos esperar uma relação linear entre o ruído de intensidade e a tensão medida nos detectores. A figura 2.9 é a verificação direta das expressões (2.6.9) e (2.6.10) respectivamente. Observou-se uma relação
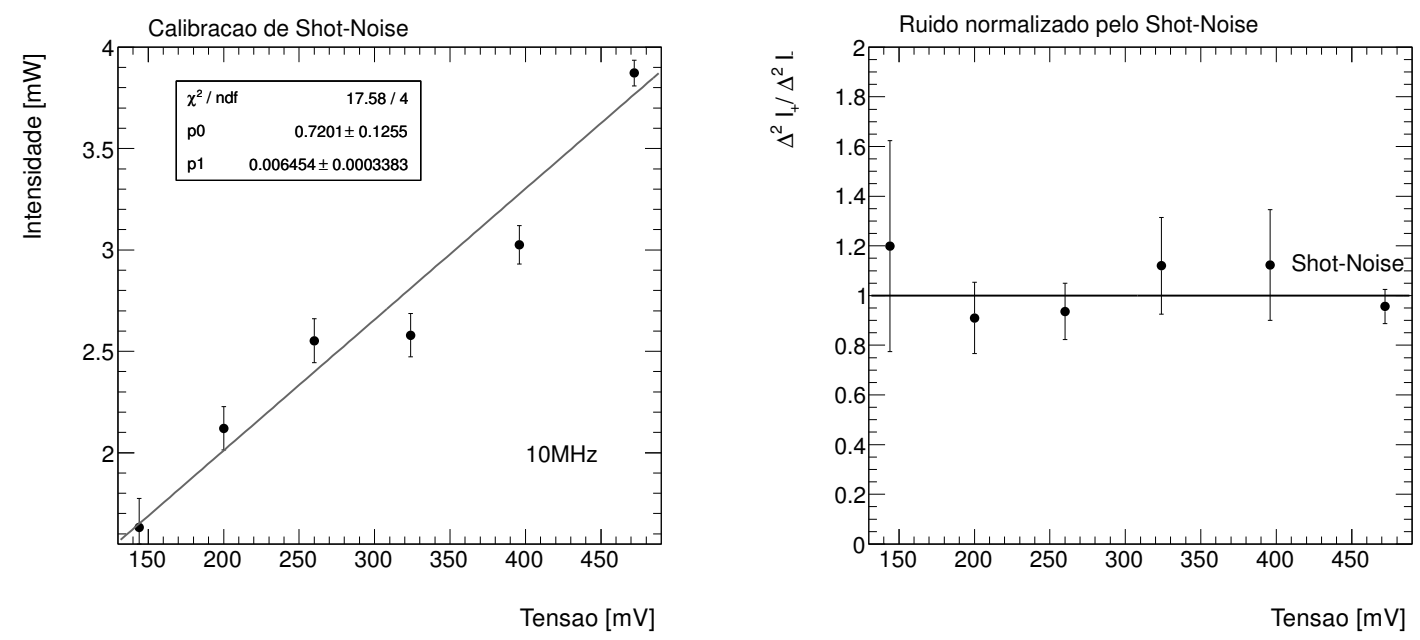

Figura 2.9: Calibração de shot-noise usando vácuo e diretamente a luz do laser. Medida feita em $5 \mathrm{MHz}$.

linear entre a intensidade do feixe de luz com a tensão no detector, em frequências até $80 \mathrm{MHz}$. Para frequências acima de $80 \mathrm{MHz}$ os detectores acrescentam ruído e a eletrônica é incapaz de realizar a subtração eficientemente. Em medidas envolvendo ruídos quânticos é importante estabelecer o nível de shot-noise precisamente. O ajuste linear dos dados permitiu determinar o ganho dos detectores. A razão entre o ruído da soma e da subtração é compatível com um, indicando pouco ruído clássico do laser. 



\section{Capítulo 3}

\section{Fibras ópticas}

Neste capítulo faremos um breve introdução sobre fibras ópticas, uma descrição física de suas propriedades. Estaremos particularmente interessados nas propriedades de não linearidade óptica, atribuindo a esta, a responsabilidade na geração de mistura de quatro ondas. Construiremos a Hamiltoniana de interação a partir da resposta do meio a um campo elétrico aplicado. Sendo a fibra óptica um meio amorfo, a primeira resposta não linear é um termo proporcional ao campo elétrico ao cubo. Veremos que, aplicada a condição de conservação da energia, obtemos uma Hamiltoniana em termos quadráticos nos operadores de criação e aniquilação do campo de bombeio, e operadores de aniquilação e criação bilineares nos modos gerados, a desejada Hamiltoniana de mistura de quatro ondas.

Em seguida, escolhendo uma relação de fase adequada entre os campos ao se propagarem pelo acoplador de onda evanescente, obtemos um conjunto de equações que se assemelham às transformações de beam-splitter. Impondo condições de contorno circulares, obtemos que as equações dos campos podem ser escritas por um processo interativo, levando a uma condição de cavidade. Para altas finesses, obtemos definições para uma cavidade de fibra que são equivalentes àquelas para uma cavidade Fabry-Perot. Por fim, faremos uma breve introdução sobre o processo de mistura de quatro ondas em fibras.

\subsection{Introdução}

O processo de geração de estados comprimidos envolve a interação da luz com um meio material. Em consequência da fraca interação não linear responsável pela geração destes estados, foi proposta a realização da mistura de quatro ondas não degenerada em fibras, como meio de 
prolongar a interação [25]. Neste contexto, o processo de mistura de quatro ondas é iniciado com a interação de dois fótons do campo de bombeio $\omega_{1}$ e $\omega_{2}$, originando outros dois fótons, denominados de sinal $\omega_{3}$, e complementar $\omega_{4}$, de tal forma que a relação de conservação da energia é obedecida $\omega_{1}+\omega_{2}=\omega_{3}+\omega_{4}$.
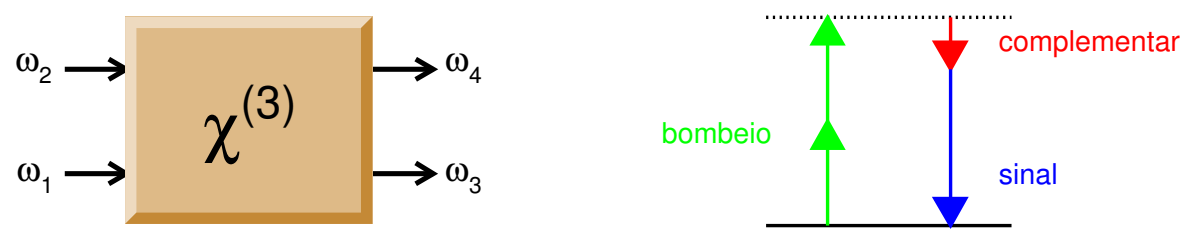

Figura 3.1: Mistura de quatro ondas degenerado. O processo de 4WM envolve a conversão de dois fótons do bombeio em um fóton do sinal e outro do complementar.

O processo de mistura de quatro ondas surge em consequência da não linearidade da fibra que está associada com a susceptibilidade de terceira ordem $\chi^{(3)}$, também conhecida como não linearidade Kerr. Uma forma de introduzir isso é através do índice de refração não linear $n_{2}$. De fato, na presença de um campo de bombeio intenso, o índice de refração da fibra é modificado segundo a relação

$$
n=n_{0}+n_{2} I,
$$

onde $n_{0}$ é o índice de refração da fibra quando a intensidade do campo é fraca, e $n$ é o índice de refração para campos fortes. Uma vez que a intensidade dos campos podem ser diferentes na fibra, e como o índice de refração está associado com a velocidade de propagação do campo no meio material, o campo de bombeio pode se propagar ao longo da fibra com uma velocidade diferente dos campos sinal e complementar, implicando em uma diferença de fase entres eles. O termo $n_{2}$ é devido principalmente ao movimento anarmônico dos elétrons como resposta ao campo aplicado. Em razão da pequena massa dos elétrons, estes respondem rapidamente às mudanças na intensidade do campo. Como resultado, pode-se considerar que a fibra tem uma resposta instantânea à intensidade do campo propagante. Devido a isto, no caso em que dois campos intensos de bombeio $\omega_{1}$ e $\omega_{2}$ se propagam na fibra, a intensidade será descrita pelas frequências $\pm \omega_{1}$ e $\pm \omega_{2}$. Se um terceiro campo com frequência $\omega_{3}$ é acoplado à mesma fibra, este 
sofrerá modulação temporal de fase devido à resposta dos elétrons ao bombeio. Portanto haverá um quarto campo cuja intensidade será descrita pelas frequências $\pm \omega_{1}, \pm \omega_{2}$ e $\pm \omega_{3}$. Este novo campo é dito ser proveniente da mistura de quatro ondas, por existir na fibra quatro campos com frequências $\omega_{1}, \omega_{2}, \omega_{3}$ e outro com combinações das frequências $\omega_{1}, \omega_{2}$ e $\omega_{3}$.

Outra forma de expressar a consequência do movimento anarmônico dos elétrons é através da polarização induzida pelos momentos de dipolo elétrico, que deixa de ser linear com o campo e passa a ser representada segundo a expressão [2]

$$
\mathbf{P}(\mathbf{r}, t)=\varepsilon_{0}\left[\chi^{(1)}(t) \otimes \mathbf{E}(\mathbf{r}, t)+\chi^{(2)}(t) \otimes \mathbf{E E}(\mathbf{r}, t)+\chi^{(3)}(t) \otimes \mathbf{E E E}(\mathbf{r}, t)\right],
$$

onde $\varepsilon_{0}$ é a permissividade do vácuo e $\chi^{(j)} \operatorname{com} j=1,2,3$ é um tensor de ordem $j+1$. A susceptibilidade linear $\chi^{(1)}$ é o termo dominante da polarização, e está relacionado com o índice de refração $n$ e o termo de atenuação $\alpha$. Os termos de ordem par da susceptibilidade está associado com processos não lineares que levam à geração de segundo harmônico e soma e subtração de frequências. O termo de segunda ordem é significativo apenas para materiais que não apresentam simetria de inversão ao nível molecular. Para fibras feitas com $\mathrm{SiO}_{2}$, material amorfo que apresenta simetria de inversão, o termo de segunda ordem é desprezível e portanto deve-se considerar apenas os efeitos de $\chi^{(3)}$.

Os parâmetros relevantes de uma fibra óptica são: o índice de refração, $n=1,45$ para sílica, o fator de atenuação, $\alpha=0,5 \mathrm{~km}^{-1}$, e a susceptibilidade não linear, $\chi^{(3)}$, que depende do modelo da fibra. A frequência do laser é assumida como sendo distante de qualquer transição atômica do estado fundamental, caso contrário, a quantidade de estados atômicos excitados passaria a ser significativo, e como consequência, haveria um acréscimo de ruído ao campo.

Como qualquer outro fenômeno eletromagnético clássico, a propagação dos campos na fibra pode ser descrita a partir das equações de Maxwell [22]

$$
\operatorname{div} \mathbf{B}=0, \quad \operatorname{rot} \mathbf{E}=-\partial_{t} \mathbf{B}, \quad \operatorname{div} \mathbf{D}=0, \quad \operatorname{rot} \mathbf{H}=\partial_{t} \mathbf{D}
$$

Uma das características da propagação do campo em fibras são as ausências de cargas elétricas livres e densidade de corrente. A equação de Maxewell pode ser usada para obter a equação de onda que descreve a propagação dos campos na fibra.

$$
\Delta \mathbf{E}(\mathbf{r}, t)=-\frac{1}{c^{2}} \frac{\partial^{2} \mathbf{E}(\mathbf{r}, t)}{\partial t^{2}}-\mu_{0} \frac{\partial^{2} \mathbf{P}(\mathbf{r}, t)}{\partial t^{2}}
$$

onde $\mu_{0}$ é a permeabilidade do vácuo, e usamos a relação $\mu_{0} \epsilon_{0}=1 / c^{2}$. Quando a frequência óptica dos campos propagantes está próxima da frequência de ressonância do meio material, um tratamento quântico da resposta do meio à presença dos campos eletromagnéticos é necessário. 
Longe dessa condição, uma resposta fenomenológica, como a descrita pela equação (3.1.2), pode ser usada. Isto define uma faixa de comprimentos de onda que vai de $0,5 \mu \mathrm{m}$ à $2 \mu \mathrm{m}$, que é o intervalo mais usado em análises de não linearidade em fibras.

O efeito da não linearidade Kerr é relativamente fraco em fibras de sílica. Portanto, podemos tratar as equações considerando como efeito principal o termo linear da polarização, e tratar a resposta não linear como um processo perturbativo. Na análise linear, as equações de Maxwell são lineares com os campos. Este fato permite desenvolver a equação de onda (3.1.4) no espaço de frequências, definindo a transformada de Fourier do campo elétrico. A vantagem do tratamento no espaço de Fourier reside na possibilidade de analisar o efeito da fibra sobre os campos, no domínio da frequência. Neste domínio, a equação de onda (3.1.4) se torna

$$
\Delta \mathbf{E}(\mathbf{r}, \omega)-\epsilon(\omega) \frac{\omega^{2}}{c^{2}} \mathbf{E}(\mathbf{r}, \omega)=0
$$

onde definimos a constante dielétrica dependente da frequência $\epsilon(\omega)=1+\tilde{\chi}^{(1)}(\omega)$, e $\tilde{\chi}^{(1)}(\omega)$ é a transformada de Fourier de $\chi^{(1)}(t)$. Em geral, $\tilde{\chi}^{(1)}(\omega)$ é um número complexo, consequentemente, $\epsilon(\omega)$ também o é. Sua parte real e imaginária podem ser relacionadas com o índice de refração $n$ e o coeficiente de absorção $\alpha$, por $\epsilon=(n+i \alpha c / 2 \omega)^{2}$. Inserindo esta relação em (3.1.5), obtemos

$$
\begin{gathered}
n(\omega)=1+\frac{1}{2} \Re\left[\tilde{\chi}^{(1)}(\omega)\right], \\
\alpha(\omega)=\frac{\omega}{n c} \Im\left[\tilde{\chi}^{(1)}(\omega)\right] .
\end{gathered}
$$

Para um feixe de bombeio com frequência $\omega_{1}=1 \times 10^{15} \mathrm{~Hz}$ acoplado a uma fibra padrão com os parâmetros acima definidos, obtemos $\Re\left(\chi^{(1)}\right)=0,9$ e $\Im\left(\chi^{(1)}\right)=2 \times 10^{-10}$.

A não linearidade Kerr depende fortemente da configuração física da fibra, como sua composição material e estrutura geométrica. Além disso, a resposta de $\chi^{(3)}$ pode ser diferente para cada campo propagante. De maneira mais geral ela pode depender tanto da frequência como da polarização do campo. No caso em que os quatro campos não estão muito distanciados em frequências, a dependência com relação a esta pode ser desprezada.

Uma fibra mono-modo convencional, não birrefringente, suporta campos com polarização linear ou circular. No 4WM degenerado, por simetria, se o campo de bombeio possui polarização linear, o sinal e complementar também o terão, podendo ser paralela ou perpendicular ao campo de bombeio. Caso a polarização do bombeio seja circular, o sinal e complementar terão polarização circular, no mesmo sentido, ou no sentido inverso. Portanto é uma boa aproximação considerar que a magnitude da não linearidade Kerr seja constante para todos os campos, mesmo possuindo uma dependência com a polarização, desde que a fibra consiga manter a polarização dos campos ao longo da propagação. Tipicamente em fibras convencionais de sílica, a magnitude da não 
linearidade Kerr é da ordem de $\chi^{(3)}=4 \times 10^{-20}$ em unidades de mks.

É comum, ao trabalhar com processos não lineares em fibras definir o fator de não linearidade $\kappa$, que pode ser escrito como [2]

$$
\kappa=\frac{n_{2} \omega_{e f}}{c A_{e f}} .
$$

O parâmetro $\kappa$ contém informações a respeito da não linearidade da fibra, através de $n_{2}$, do modo de propagação, através da área efetiva $A_{e f}$, e também dos acoplamentos dos campos, através de uma frequência efetiva $\omega_{e f}$, dada por uma combinação dos modos propagantes [35]. Para um modo Gaussiano, a área efetiva é duas vezes a área do feixe Gaussiano propagante com a mesma intensidade e potência. Para se ter uma idéia da magnitude de $\kappa$ é comum considerar a frequência e a área efetiva como sendo a frequência do campo de bombeio e a área do núcleo da fibra, respectivamente.

\subsection{A Hamiltoniana não linear}

Em uma fibra birrefringente, todos os campos linearmente polarizados possuem a mesma direção de polarização, portanto podemos ignorar a caráter vetorial da expressão de polarização do meio (3.1.2), e tratá-la como escalar.

A densidade de energia associada ao campo é escrita como $U=U_{L}+U_{N L}$, onde $U_{L}$ provem do termo linear da polarização e resulta no termo quadrático da energia, existindo mesmo na ausência de um meio não linear. O termo $U_{N L}$ é devido à interação não linear da polarização. Ambas energias são calculadas de acordo com $P=-d U / d E$ [56], temos portanto

$$
U_{L}=-\varepsilon_{0} \frac{1+\chi^{(1)}}{2} E^{2} ; U_{N L}=-\varepsilon_{0} \frac{\chi^{(3)}}{4} E^{4} .
$$

A Hamiltoniana do processo não linear é então obtida integrando, por todo o volume de interação, a energia responsável pelo processo.

Partimos do pressuposto da existência de quatro ondas monocromáticas com frequências $\omega_{l}$, $\operatorname{com} \imath=1,2,3$ e 4, e condicionamos as frequências à conservação de energia $\omega_{1}+\omega_{2}=\omega_{3}+\omega_{4}$. A transição para a mecânica quântica é realizada introduzindo os operadores $\hat{a}_{\imath}^{\dagger}\left(\omega_{\imath}\right)$ e $\hat{a}_{\imath}\left(\omega_{\imath}\right)$ como amplitudes de Fourier dos operadores de criação e aniquilação $\hat{a}_{l}^{\dagger}(z)$ e $\hat{a}_{\imath}(z)$ respectivamente, onde $z$ é a direção longitudinal da fibra [35]. Estes operadores devem obedecer a regra de comutação $\left[\hat{a}_{\imath}\left(\omega_{\imath}\right), \hat{a}_{j}^{\dagger}\left(\omega_{\jmath}\right)\right]=\delta_{\imath, \jmath}$. Assim sendo, podemos escrevemos o campo elétrico total 
como

$$
E(\mathbf{r}, t)=\sum_{\imath=1}^{4} N_{\imath} \hat{a}_{\imath}\left(\omega_{\imath}\right) F_{\imath}(x, y) \exp \left(i \beta_{\imath} z-i \omega_{\imath} t\right)+h . c,
$$

onde $F_{\imath}(x, y)$ descreve o perfil transversal dos campos, e iremos considerá-lo como real a partir deste ponto. O termo $\beta_{\imath}=n_{\imath} k_{\imath}$ é o módulo do vetor de onda na fibra, e o fator de normalização $N_{j}$ é escolhido convenientemente de modo que o termo quadrático da energia leve à Hamiltoniana linear $\hbar \omega_{\imath} \hat{a}_{\imath}^{\dagger} \hat{a}+1 / 2$.

A densidade de energia não linear pode então ser escrita como

$$
U_{N L}=\frac{\chi^{(3)}(x, y) \hbar^{2}}{4 \varepsilon_{0} l^{2}}\left\{\sum_{\imath=1}^{4} \sqrt{\frac{\omega_{\imath}}{R_{\imath}}}\left[\hat{a}_{\imath}\left(\omega_{\imath}\right) \exp \left(i \beta_{\imath} z-i \omega_{\imath} t\right)+\hat{a}_{\imath}^{\dagger}\left(\omega_{\imath}\right) \exp \left(-i \beta_{\imath} z+i \omega_{\imath} t\right)\right] F_{\imath}(x, y)\right\}{ }^{4},
$$

onde $l$ é o comprimento da fibra e $R_{\imath} \equiv \int_{x, y}\left[n_{\imath}(x, y) F_{\imath}(x, y)\right]^{2} d x d y$. Os limites de integração de $R_{\imath}$ em $x$ e $y$ devem ser estendidos até os domínios dos modos espaciais. Em geral, assume-se que os domínios dos modos espaciais são limitados e estende-se a integração até $-\infty$ e $+\infty$. A parte não linear da Hamiltoniana é então obtida integrando $U_{N L}$ ao longo de todo volume de interação do campo com a fibra.

Estamos interessados nos termos que oscilam lentamente na expressão (3.2.3), isto é, que não dependam das frequências. Dentro desta categoria existem diferentes combinações de operadores que possibilitam eliminar a dependência na frequência.

Combinações dos tipos $\hat{a}_{\imath}^{\dagger} \hat{a}_{\imath}^{\dagger} \hat{a}_{\imath} \hat{a}_{\imath}$ e $\hat{a}_{\imath}^{\dagger} \hat{a}_{\imath}^{\dagger} \hat{a}_{\jmath} \hat{a}_{\jmath}$, com $\imath \neq \jmath$, dão origem a efeitos conhecidos respectivamente por auto modulação de fase e modulação de fase cruzada.

A imposição da lei de conservação da energia permite escrever mais uma combinação de operadores que elimina a dependência na frequência, uma vez que $\omega_{1}+\omega_{2}-\omega_{3}-\omega_{4}=0$. A combinação destes termos levam à Hamiltoniana do 4WM

$$
\hat{H}_{4 W M}=\frac{4 ! \hbar^{2} \chi^{(3)}}{4 \varepsilon_{0} l^{2}} \frac{\sqrt{\omega_{1}^{2} \omega_{3} \omega_{4}}}{n_{1}^{2} n_{3} n_{4}}\left[\int_{0}^{l} \exp (-i \Delta \beta z) d z\right] f \hat{a}_{1}^{2} \hat{a}_{3}^{\dagger} \hat{a}_{4}^{\dagger}+h . c,
$$

onde 4 ! são as possíveis combinações dos operadores $\hat{a}_{1}^{2} \hat{a}_{3}^{\dagger} \hat{a}_{4}^{\dagger}$, pois todos comutam entre si. O argumento da exponencial $\Delta \beta=\beta_{3}+\beta_{4}-2 \beta_{1}$ contabiliza o acordo de fase dos campos e

$$
f=\frac{\int_{x, y} F_{1}^{2}(x, y) F_{3}(x, y) F_{4}(x, y) d x d y}{\sqrt{\left[\int_{x, y} F_{1}^{2}(x, y) d x d y\right]^{2} \int_{x, y} F_{3}^{2}(x, y) d x d y \int_{x, y} F_{4}^{2}(x, y) d x d y}}
$$


é responsável pelo acordo de modo.

A forma da Hamiltoniana contém combinações dos operadores de criação e aniquilação que revelam ao nível mais fundamental o processo de interação entre quatro fótons dos campos. $\mathrm{O}$ operador $\hat{a}_{1}^{2}$ indica a destruição simultânea de dois fótons do bombeio para a criação, também simultânea, dos fótons do sinal e complementar, representado pelos operadores $\hat{a}_{3}^{\dagger}$ e $\hat{a}_{4}^{\dagger}$. O processo inverso, a destruição dos fótons do sinal e complementar para a criação de dois fótons do bombeio, ocorre no Hermitiano conjugado da Hamiltoniana.

Em ambos os casos, dois fótons são destruídos para o surgimento de dois outros fótons. Portanto em um processo de mistura de quatro ondas, o número de fótons é conservado.

É comum em processos paramétricos considerar o campo de bombeio intenso, de maneira que não sofra depleção devido aos fótons gerados nas bandas laterais. Nessa condição considera-se o campo de bombeio clássico, isto é, ele deixa de ser representado por operadores e passa a ser escrito como números complexos. A vantagem deste tratamento resulta na simplificação das equações de dinâmica do processo, desacoplando os campos de sinal e complementar dos campos de bombeio.

As flutuações nas bandas laterais se tornam correlacionadas pelo processo de mistura de quatro ondas, e um deslocamento de fase do bombeio com relação às bandas laterais podem revelar compressão nas quadraturas. Uma maneira de intensificar o processo de compressão é fazendo uso de cavidades ópticas em fibras. Este sistema foi inicialmente proposto em 1986 com a finalidade de aumentar a interação do campo com o meio material [43].

\subsection{Cavidades em fibras}

A cavidade em fibras é baseada no acoplamento por onda evanescente em guias de onda [20]. O dispositivo consiste basicamente em um "loop" de fibra com a aproximação dos núcleos, onde uma fração do campo de saída é acoplado novamente com o campo de entrada por um acoplador comercial. O processo de acoplamento por onda evanescente provém dos princípios da mecânica quântica e das equações de Maxwell, em particular, dos estudos das funções de ondas em poços de potenciais. A exigência da continuidade da função de onda nos limites do potencial tem como consequência o tunelamento dela na direção interior ao potencial.

O acoplamento em fibras ópticas por onda evanescente exige a aproximação dos núcleos das duas fibras a uma distância curta o suficiente para que a taxa de decaimento do campo não o impeça de atingir a interface dos meios. O que se faz normalmente é esquentar e esticar os núcleos das fibras, no sentido de reduzir o diâmetro do núcleo e aumentar a região de onda 
evanescente. Uma representação pictórica do acoplador e a geometria da cavidade em anel pode ser observada na figura 3.2.

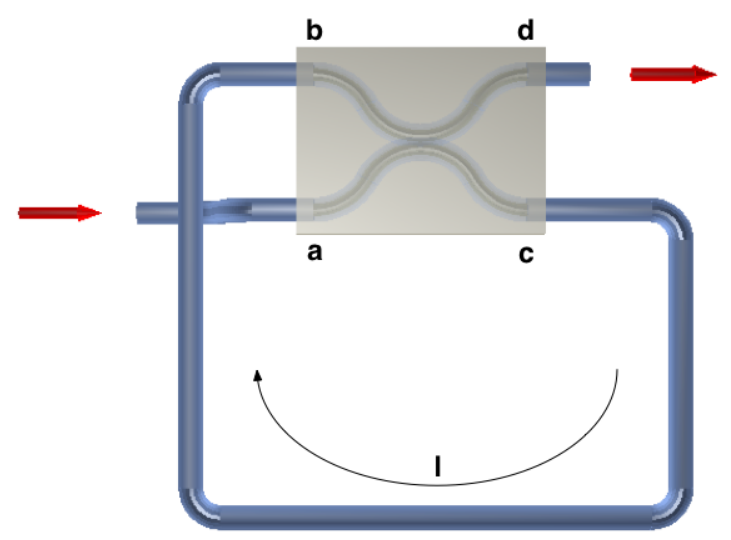

Figura 3.2: Representação pictórica da cavidade em anel. A aproximação dos núcleos no acoplador permite o acoplamento por onda evanescente dos campos. O comprimento da fibra no loop é $l$.

O acoplador é constituído de quatro portas $a, b, c$ e $d$. O campo de entrada é acoplado à porta $a$. Interferência construtiva deve ser estabelecida entre os campos acoplados com a porta $c$ provenientes das portas $a$ e $b$. Uma pequena fração do campo em $b$, acoplado com a porta $d$, deve sofrer interferência destrutiva com o campo acoplado com as portas $a$ e $d$. A intensidade do campo circulante irá aumentar até que o equilíbrio seja atingido, ou seja, até que as perdas dentro do loop, igualem à potência de entrada na porta $a$.

O comprimento do loop é $l$ e pode ser constituído por uma fibra birrefringente ou não. Em ambos os casos, para conseguir a máxima interferência no acoplamento, a polarização do campo na porta $b$ deve igual à polarização na porta $a$. O ajuste da polarização é feito com um controlador de polarização na porta $b$.

A intensidade campo de entrada na porta $a$ é acoplado com a porta $d$ com uma eficiência $\eta$. A mesma eficiência é considerada no acoplamento entre as portas $b$ e $c$. Comercialmente é possível 
encontrar acopladores com valores de $\eta$ indo de 0,50 até 0,99.

O acoplador e o loop de fibra adicionam perdas que atenuam o campo intracavidade. A perda no acoplador pode variar de $0,5 \mathrm{~dB}$ a $1 \mathrm{~dB}$, sendo predominantemente causada por perda de inserção e pelos conectores que fazem a ligação entre o acoplador e a fibra. As perdas na fibra são devidas principalmente à absorção e espalhamento.

As amplitudes complexas dos campos, após o acoplamento, se relacionam por

$$
\begin{aligned}
& E_{c}=\sqrt{1-\gamma_{0}}\left[\exp \left(i \Phi_{a c}\right) \sqrt{1-\eta} E_{a}+\exp \left(i \Phi_{b c}\right) \sqrt{\eta} E_{b}\right] \\
& E_{d}=\sqrt{1-\gamma_{0}}\left[\exp \left(i \Phi_{a d}\right) \sqrt{\eta} E_{a}+\exp \left(i \Phi_{b d}\right) \sqrt{1-\eta} E_{b}\right]
\end{aligned}
$$

onde $\gamma_{0}$ amarra as perdas nos acopladores e $\exp \left(i \Phi_{\imath \jmath}\right)$ é a fase acrescida pelo acoplador ao campo quando este é transmitido da porta $\imath$ para a porta $\jmath$.

A condição de conservação da energia nos leva a impor que os argumentos das exponenciais da soma dos módulos ao quadrado das (3.3.1) se anule, levando a uma relação entre as fases

$$
\Phi_{a c}+\Phi_{b d}-\Phi_{b c}-\Phi_{a d}= \pm \pi .
$$

Existe uma diversidade de combinações possíveis na escolha das fases, determinando o funcionamento do acoplador. Uma escolha possível é fazer $\Phi_{a d}=\pi$ e todas as outras iguais a zero. Com esta escolha, o acoplador funciona como um beam-splitter de refletividade $\eta$, e as transformações dos campos se assemelham às (2.6.1).

O campo $E_{c}$ recebe um atraso de fase de $\Phi=n \omega l / c$ ao percorrer o loop de fibra com comprimento $l$. Além disso, o campo está sujeito a absorção dos fótons pelos átomos da fibra, e também a processos de espalhamento, atenuando o campo ao longo do loop. Portanto podemos escrever

$$
E_{b}=\exp (i \Phi) \exp \left(-\alpha_{0} l\right) E_{c},
$$

onde $\alpha_{0}$ é responsável por contabilizar a atenuação na fibra. Atualmente as fibras são construídas com um fator de atenuação relativamente baixo $\alpha_{0} \sim-0,5 \mathrm{~dB} / \mathrm{km}$, que para cavidades curtas significa perdas pequenas quando comparadas com as perdas no acoplador.

Ao escrever o campo de entrada na porta $b$ em função do campo de saída na porta $c$, estabelecemos uma configuração de cavidade no acoplador. A consequência disto é que podemos escrever as equações de transformação dos campos (3.3.1) como um processo iterativo. As amplitudes dos campos circulante e de saída na frequência $\omega$ são

$$
E_{c}=\frac{\sqrt{(1-\eta)\left(1-\gamma_{0}\right)}}{1-\sqrt{\eta\left(1-\gamma_{0}\right)} \exp \left(-\alpha_{0} l+i \Phi\right)} E_{a}
$$




$$
E_{d}=-\sqrt{\eta\left(1-\gamma_{0}\right)}\left[1-\frac{(1-\eta) \sqrt{\left(1-\gamma_{0}\right) / \eta} \exp \left(-\alpha_{0} l+i \Phi\right)}{1-\sqrt{\eta\left(1-\gamma_{0}\right)} \exp \left(-\alpha_{0} l+i \Phi\right)}\right] E_{a},
$$

onde $\sqrt{\eta\left(1-\gamma_{0}\right)} \exp \left(-\alpha_{0} l+i \Phi\right)$ é o efeito da cavidade sobre o campo circulante a cada volta. Para uma cavidade ressonante exigimos que a parte real e imaginária do campo $E_{d}$ se anulem, levando a duas condições de ressonância. A primeira diz que a fase $\Phi$ deve ser um múltiplo inteiro de $\pi$, cuja implicação diz que a cavidade irá passar pela condição de ressonância sempre que o caminho óptico da fibra $\mathrm{nl}$ for alterado em meio comprimento de onda.

A segunda condição determina o valor do parâmetro do acoplador $\eta$ para a máxima ressonância, isto é, sem campo refletido (acoplamento crítico)

$$
\eta_{r}=\left(1-\gamma_{0}\right) \exp \left(-2 \alpha_{0} l\right)
$$

Note que $1-\eta_{r}$ são as perdas na cavidade em uma volta. A figura 3.3 mostra a intensidade do campo refletido para diferentes acoplamentos. Na condição de ressonância o campo refletido é

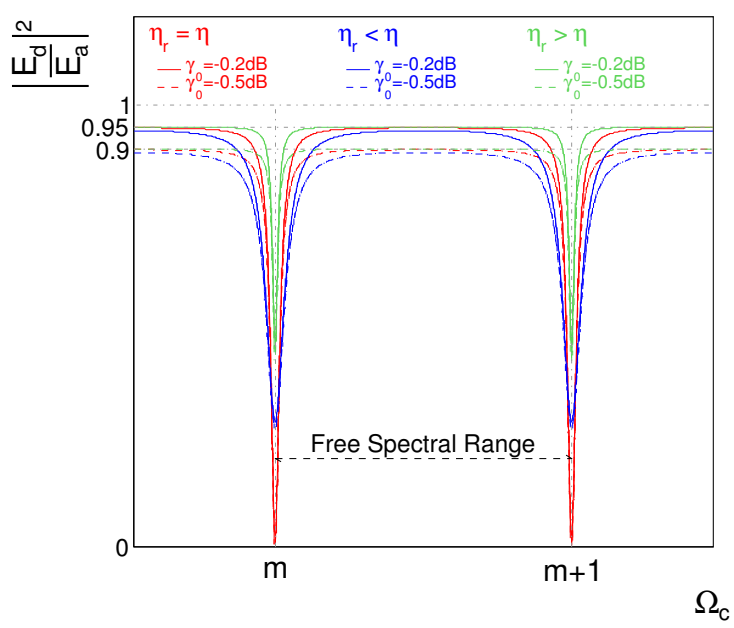

Figura 3.3: Curva teórica para a intensidade do campo refletido pela cavidade. A eficiência $\eta$ é de 0,9 e as perdas nos acopladores de $0,2 \mathrm{~dB}$ e $0,5 \mathrm{~dB}$. A figura também compara os casos onde $\eta_{r}=0,7<\eta$, acoplamento sub-critico, e $\eta_{r}=0.95>\eta$, acoplamento super-critico.

mínimo e obtemos a máxima intensidade do campo circulante

$$
\left|\frac{E_{c}}{E_{a}}\right|_{\max }^{2}=\frac{(1-\eta)\left(1-\gamma_{0}\right)}{\left(1-\sqrt{\eta \eta_{r}}\right)^{2}}
$$


O comprimento do caminho óptico é $n l$ e o intervalo espectral livre - Free Spectral Range - é definido como

$$
\omega_{c}=\frac{2 \pi c}{n l}
$$

Definimos a largura de linha da cavidade como a largura à meia altura dos picos de transmissão. Para uma eficiência próxima da unidade obtemos

$$
\delta \omega_{c}=\omega_{c} \frac{\left(1-\sqrt{\eta \eta_{r}}\right)}{\pi\left(\eta \eta_{r}\right)^{1 / 4}}
$$

e definimos a finesse da seguinte forma

$$
F=\frac{\omega_{c}}{\delta \omega_{c}}=\frac{\pi\left(\eta \eta_{r}\right)^{1 / 4}}{\left(1-\sqrt{\eta \eta_{r}}\right)}
$$

Devemos notar que, no regime de alta finesse, obtemos para uma cavidade em anel construída com fibra óptica, a mesma expressão da finesse de uma cavidade do tipo Fabry-Perot (2.5.1), com $\eta$ e $\eta_{r}$ fazendo os papéis das refletâncias dos espelhos de entrada e do segundo espelho respectivamente.

O tempo de decaimento da cavidade é o tempo em que demora a dissipação da energia no interior da cavidade quando o campo de entrada é desligado

$$
\tau=\frac{n l / c}{\delta}
$$

onde $\delta$ são os meios de dissipação da energia a cada volta. Estes meios incluem as perdas por dispersão e absorção que ocorrem na fibra, assim como as perdas e transmissão nos acopladores

$$
\tau=\frac{n l / c}{\left(1-\eta_{r}\right)+(1-\eta)}
$$

A perda no acoplador é de aproximadamente $15 \%$ e é causada principalmente por perdas de inserção. Estas perdas são muito maiores que as que ocorrem na fibra devido a atenuação. O tempo máximo de permanência dos fótons em um loop de fibra de $60 \mathrm{~m}$ é de $1,5 \mu$ s e ocorre quando $\eta=\eta_{r}=0,9$. Portanto um laser com comprimento de coerência superior a $c \tau$ pode produzir efeitos de interferência.

O comprimento do loop de fibra que empregamos é de $60 \mathrm{~m}$ e parte dele é enrolado em uma estrutura de PZT - piezoeletric actuator - que pode expandir esticando a fibra e com isso alterando o caminho óptico $n l$, causando uma modulação de fase no campo. Um controlador de polarização é utilizado na entrada da porta $b$ para maximizar a interferência no acoplador. $\mathrm{O}$ laser da RIO com comprimento de coerência longo, largura de linha de aproximadamente $3 \mathrm{KHz}$, 


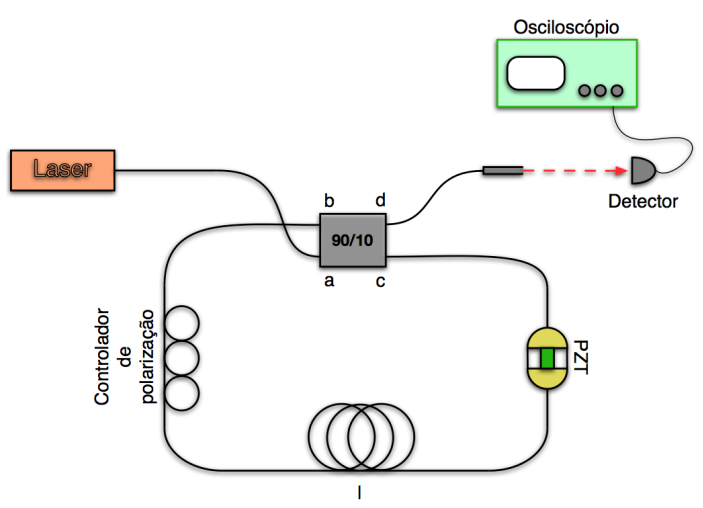

Figura 3.4: Representação pictórica da montagem de uma cavidade oscilante com fibras. Um laser estável com largura de linha estreita é necessário para enxergar as ressonâncias da cavidade. O campo do laser é acoplado com o acoplador da Thorlabs de eficiência $\eta=90 \%$. Parte da fibra que compõe o interior da cavidade é enrolado numa estrutura de PZT da forma cilíndrica em um número de 4 à 6 voltas. A outra ponta da fibra passa por um controlador de polarização para em seguida ser acoplado com a porta $b$. Por fim, o feixe de saída da cavidade é monitorado pelo detector PDA10CS da Thorlabs.

centrado em $\lambda=1,56 \mu \mathrm{m}$ foi utilizado como fonte de luz. Uma tensão de baixa frequência, triangular, centrada em $30 \mathrm{~V}$, com amplitude pico a pico de $10 \mathrm{~V}$ foi aplicada ao PZT possibilitando a varredura dos picos da cavidade. O sinal detectado pelo fotodiodo na saída da porta $d$ pode ser observado na figura 3.5

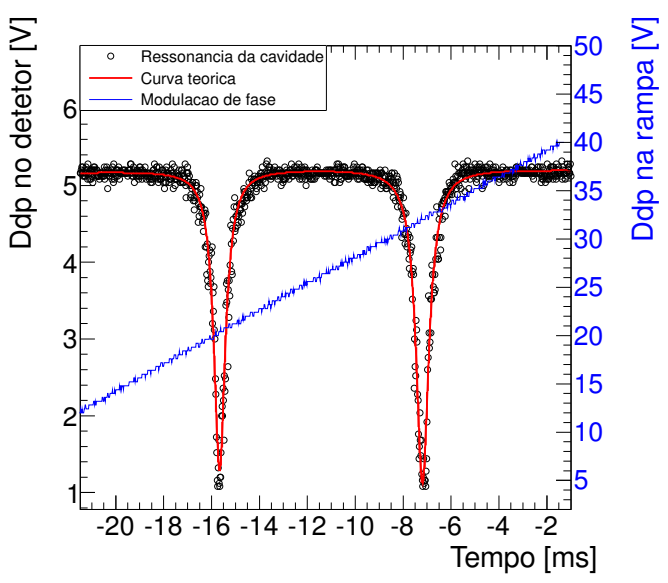

Figura 3.5: Uma tensão variável no forma de rampa de aproximadamente $30 \mathrm{~V}$ é aplicado a um modulador de fase no interior da cavidade. A partir da intensidade do campo na saída medimos uma finesse de 14,72 . 
Dos dados experimentais acima obtemos uma finesse de 14,72(19). A partir do valor da finesse, estimamos pela equação (3.3.10) as perdas intracavidade em aproximadamente $25 \%$. A atenuação da fibra é precisamente medida, e em 60 metros de comprimento representa uma perda da ordem de $5,8 \%\left(2 \alpha_{0} l=0,06\right)$, que implica em uma perda de inserção mais conexão de aproximadamente $19,2 \%$.

\subsection{Efeito da cavidade}

A cavidade óptica exerce um papel fundamental no tratamento quântico dos campos, fornecendo uma fronteira entre o sistema de estudo em questão e o meio exterior. A influência externa é tratada como um reservatório térmico, no sentido em que o sistema não é capaz de perturbá-lo devido à interação mútua. Por sua vez, a ação do reservatório sobre o sistema é a causa do ruído clássico e quântico.

O reservatório térmico é tratado como um conjunto de osciladores harmônicos com um espectro denso de frequências de oscilação. Esta abordagem é conveniente uma vez que também modelamos o campo eletromagnético como um conjunto de osciladores harmônicos, no entanto, esta não é o único tratamento possível [15]. Algumas considerações devem ser comentadas neste tipo de interação, as quais são: o acoplamento do banho térmico com o sistema deve ser linear nos operadores quânticos dos osciladores, e a constante de acoplamento do sistema com os operadores do banho deve ser uma função "comportada" na frequência.

Neste contexto, a cavidade fornece uma porta de interação entre o sinal e o banho térmico, cuja ação sobre o sistema é incluir termos aparentemente aleatórios, descritos por variáveis estocásticas. Este tipo de problema foi inicialmente tratado por Langevin, cujas equações levam o seu nome [15].

A cavidade então descreve um processo de entrada e saída, no qual o acoplamento com o banho térmico adiciona ruído de entrada ao sistema, e este irradia o ruído modificado. Desta forma, o campo de entrada é composto de um sinal, que é produzido por uma fonte de luz, um ruído coerente que é devido às flutuações da fonte de luz, e o ruído proveniente do banho térmico, que em óptica quântica é denominando de ruído de vácuo.

O ruído de vácuo existe mesmo na ausência do sinal e das flutuações térmicas, isto é, em um ambiente isolado e com temperatura em zero absoluto. Este ruído é caracterizado por possuir valor médio nulo, mas valor médio quadrático diferente de zero. Matematicamente, o efeito das flutuações de vácuo é garantir a existência de um termo estocástico de entrada, e um termo dissipativo. Estes termos sustentam a existência da relação de comutação dos operado- 
res, assegurando a validade da mecânica quântica, mais precisamente a relação de incerteza de Heisenberg.

Para uma cavidade construída com fibra óptica, os modos do campo eletromagnético interior à cavidade serão ditados pelos modos da fibra, e será o modo fundamental caso a fibra seja monomodo. Se o acoplamento com o meio externo também for por meio de uma fibra mono-modo com as mesmas especificações geométricas, então um acordo de modo perfeito é estabelecido na fronteira.

Para uma cavidade em anel do tipo da descrita na seção 3.3, há apenas um acoplamento entre os campos externo e interno, e este ocorre pelo acoplador com eficiência $1-\eta$. Se a cavidade estiver em ressonância, e o campo de entrada for desligado, o número de fótons internos, dado pelo operador número $\hat{a}^{\dagger} \hat{a}$, irá decair exponencialmente através desta porta e devido às perdas espúrias

$$
n_{\text {cav }}(t)=n_{\text {cav }}(0) \exp (-2 \kappa t)
$$

onde $\kappa$ contabiliza a taxa das perdas totais na cavidade, incluindo a taxa de acoplamento dos campos. Como o fluxo de entrada dos fótons é dado apenas por intermédio do acoplador, temos

$$
\frac{d n_{c a v}}{d t}=-2 \kappa n_{c a v}+(1-\eta) N_{a},
$$

onde $N_{a}$ é o fluxo de fótons externos que chegam à porta $a$ do acoplador. Na situação estacionária, o número de fótons intracavidade é

$$
n_{\text {cav }}=\tau(1-\eta) N_{a}
$$

Vemos que na situação de equilíbrio o número interno de fótons é proporcional a $\tau$, o tempo de decaimento da cavidade.

As perdas na cavidade representam uma porta de entrada e saída de valores médios e flutuações dos campos. Portanto, a equação de dinâmica que melhor descreve a intensidade os modos internos do sistema, com uma e entrada e perdas indesejadas, pode ser escrita como [3]

$$
\frac{d\left(\hat{a}^{\dagger} \hat{a}\right)}{d t}=-2 \kappa \hat{a}^{\dagger} \hat{a}+2 \kappa_{i n} \hat{a}_{a}^{\dagger} \hat{a}_{a}+2 \kappa_{l} \hat{a}_{l}^{\dagger} \hat{a}_{l}
$$


onde os subscritos $a$ e $l$ indicam o modo do campo de entrada e as perdas espúrias respectivamente. As constantes que acompanham os operadores número são dadas por

$$
\begin{aligned}
& \kappa=\frac{c\left[(1-\eta)+\left(1-\eta_{r}\right)\right]}{2 n l}, \\
& \kappa_{i n}=\frac{c(1-\eta)}{2 n l}, \\
& \kappa_{l}=\frac{c\left(1-\eta_{r}\right)}{2 n l}
\end{aligned}
$$

e quantificam a taxa de transferencia de fótons. O primeiro termo do lado direito da expressão (3.4.4) descreve o decaimento exponencial dos modos internos na ausência de um campo de entrada. O segundo termo descreve o fluxo de entrada de fótons pela porta a na cavidade devido ao campo de sinal, e o último termo completa a entrada indesejada dos valores médios dos campos através da porta responsável pelas perdas. Podemos fazer o valor médio $\left\langle\hat{a}_{l}^{\dagger} \hat{a}_{l}\right\rangle=0$, uma vez que, para o estado de vácuo, o número de fótons pode ser desprezado, diferente do que ocorre com as flutuações. Da mesma forma como definimos a (3.4.4) para o número de fótons, podemos definir um dinâmica semelhante para o modo interno, $\hat{a}$

$$
\frac{d \hat{a}}{d t}=-\kappa \hat{a}+\sqrt{2 \kappa_{i n}} \hat{a}_{a}+\sqrt{2 \kappa_{l}} \hat{a}_{l} .
$$

A condição de contorno no acoplador para o modo refletido $\hat{a}_{d}$ é

$$
\hat{a}_{d}=\sqrt{2 \kappa_{\text {out }}} \sqrt{1-\kappa_{l}} \hat{a}-\sqrt{\eta} \hat{a}_{a},
$$

onde $\kappa_{\text {out }}$ é a taxa na qual os fótons deixam a cavidade. Uma boa aproximação é considerar que $\kappa_{\text {in }}=\kappa_{\text {out }}$ para o acoplador estudado. A solução estacionária é encontrada fazendo $d \hat{a} / d t=0$

$$
\begin{aligned}
& \hat{a}^{\dagger} \hat{a}=\frac{2 \kappa_{\text {in }}}{\kappa^{2}} \hat{a}_{a}^{\dagger} \hat{a}_{a}, \\
& \hat{a}_{d}^{\dagger} \hat{a}_{d}=\left[\frac{4 \kappa_{\text {in }} \kappa_{\text {out }}\left(1-\kappa_{l}\right)}{\kappa^{2}}+\eta\right] \hat{a}_{a}^{\dagger} \hat{a}_{a} .
\end{aligned}
$$

Obtemos os mesmos resultados clássicos com o tratamento quântico da cavidade, em termos do número de fótons. Este resultado é esperado, uma vez que número de fótons está diretamente relacionado com as intensidades dos campos (3.3.4) e (3.3.5). Contudo, resultados novos são obtidos quando fazemos o tratamento quântico das flutuações dos campos.

As flutuações dos campos podem ser tratadas em termos de suas variâncias, e a equação quântica que descreve suas evoluções é análoga à (3.4.6). A solução da equação nos fornece informações a respeito do processo de transformação das flutuações do campo de entrada. Para uma cavidade 
em ressonância, as flutuações do campo de saída dependem das flutuações do campo de entrada, e das flutuações de vácuo, que são inseridas na cavidade através da mesma porta responsável pelas perdas. No caso de uma cavidade construída com fibra óptica, implica que as flutuações do vácuo irão adicionar ruído ao campo interno pelo acoplador, e ao longo de todo o comprimento da fibra.

O tratamento é feito a partir da linearização dos campos [55], onde consideramos os operadores dos campos descritos por um valor médio, representado por um número complexo, mais um termo de flutuação em torno deste valor

$$
\hat{a}=\alpha+\delta \hat{a}
$$

Linearizando todos os modos envolvidos e substituindo em (3.4.6) temos

$$
\frac{d \delta \hat{a}}{d t}=-\kappa \delta \hat{a}+\sqrt{2 \kappa_{i n}} \delta \hat{a}_{a}+\sqrt{2 \kappa_{l}} \delta \hat{a}_{l}
$$

Somando com o complexo conjugado da equação acima, obtemos a expressão que descreve a evolução das flutuações da quadratura de amplitude dos campos

$$
\frac{d \delta \hat{X}}{d t}=-\kappa \delta \hat{X}+\sqrt{2 \kappa_{i n}} \delta \hat{X}_{a}+\sqrt{2 \kappa_{l}} \delta \hat{X}_{l}
$$

O primeiro termo desta equação descreve a evolução do ruído da quadratura de amplitude do campo intracavidade, que não é acessível. O segundo termo especifica as flutuações do campo de sinal, isto é, as flutuações do laser. Enquanto que o último termo inclui o ruído de vácuo, que é não nulo.

A condição de contorno na porta $d$ do acoplador é

$$
\delta \hat{X}_{d}=\sqrt{2 \kappa_{\text {out }}} \delta \hat{X}-\delta \hat{X}_{a}
$$

onde $\delta \hat{X}_{a}$ descreve a flutuação da quadratura de amplitude do campo que atinge a porta $a$, e $\delta \hat{X}$ descreve a flutuação do campo intracavidade.

Como vimos na seção 2.2 é interessante analisar o comportamento espectral dos ruídos, para tanto é conveniente definir a transformada de Fourier para as flutuações. Aplicando a transformada de Fourier em (3.4.11) e levando em conta a condição de contorno (3.4.12), obtemos

$$
\delta \hat{X}_{d}=\frac{\left(\sqrt{4 \kappa_{\text {in }} \kappa_{\text {out }}}-\kappa+i \Omega\right) \delta \hat{X}_{a}+\sqrt{4 \kappa_{\text {out }} \kappa_{l}} \delta \hat{X}_{l}}{\kappa-i \Omega} .
$$


Esta expressão descreve como a flutuação do laser é modificada pelo ruído de vácuo ao passar pela cavidade. O espectro das flutuações é determinado através do cálculo da variância do valor médio da quadratura

$$
\operatorname{var}\left(\hat{X}_{d}\right)=\left\langle\delta \hat{X}_{d}^{*} \delta \hat{X}_{d}\right\rangle
$$

O primeiro caso interessante acontece quando a variância do campo de entrada é limitada ao shot-noise. Neste caso obtemos

$$
\operatorname{var}\left(\hat{X}_{d}\right)=1
$$

Se todas as flutuações dos campos de entrada obedecem ao limite quântico padrão, então a cavidade atua como um instrumento passivo, isto é, a variância do campo de saída também estará limitada ao shot-noise.

Vamos analisar agora o efeito das flutuações de vácuo sobre a cavidade quando a variância do campo de entrada é diferente do shot-noise, isto é, quando o campo de entrada apresenta excesso ou redução de ruído na quadratura de amplitude. Podemos ver pela figura 3.6, que

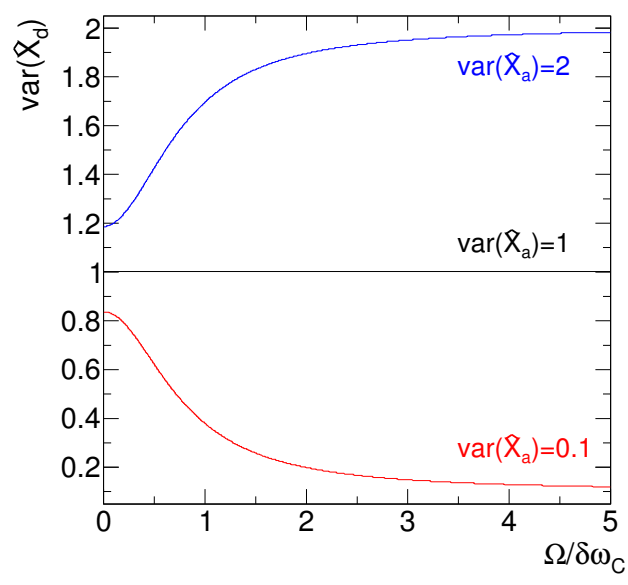

Figura 3.6: Curva teórica que explica o efeito da cavidade sobre a variância do campo de entrada devido à introdução do ruído de vácuo pelas perdas da mesma. Três casos foram considerados: quando a variância do campo de entrada é igual a 1; e quando esta apresenta excesso e redução de ruído. O parâmetros utilizados foram aqueles obtido na seção 3.3.

a variância do campo de saída depende da frequência de análise $\Omega$, como era de se esperar segundo a equação (3.4.13). Para frequências baixas, a cavidade é mais sensível ao ruído de vácuo, adicionando ruído ao campo de sinal. Enquanto que para frequências altas, a variância do campo de entrada sofre pouca influência do ruído indesejado, que é inserido pela porta das perdas incontroláveis.

É interessante notar que não temos acesso direto à variância do campo intracavidade, a descri- 
ção em termos deste campo é puramente teórica. Em muitos casos, a variância da quadratura de amplitude do campo de entrada é caracterizada como sendo própria de um estado coerente, e o processo não linear de redução ou aumento de ruído em uma das quadraturas, ocorre dentro da cavidade. Sendo assim, temos acesso apenas ao campo refletido, e este contém influências dos ruídos do campo circulante e do vácuo.

\subsection{Mistura de quatro ondas}

A mistura de quadro ondas é um processo paramétrico, no sentido em que o fenômeno não linear existente no meio material tem origem na modulação de algum parâmetro interno do meio material, tal como o índice de refração. Fundamentalmente, a explicação física da origem da modulação remete à resposta não linear dos elétrons ao meio material, quando este está sujeito a um campo intenso. Mais especificamente, a polarização induzida no meio material não é linear, mas contém termos proporcionais a potências superiores do campo elétrico. O termo relacionado com o campo elétrico ao quadrado, descrito por $\chi^{(2)}$, é responsável por processos conhecidos como soma e subtração de frequências, e geração de segundo harmônico [6]. Processos de segunda ordem não ocorre em meios isotrópicos, por isso esperamos que estes efeitos não ocorram em fibras de sílica ${ }^{1}$.

Processos não lineares de terceira ordem envolvem interações não lineares entre quatro campos, e inclui fenômenos como geração de terceiro harmônico, mistura de quatro ondas e amplificação paramétrica [2,35]. A eficiência destes efeitos requer o acordo de fase [49], que em linhas gerais, exige que todos os comprimentos de onda dos campos de interesse estejam próximos do zero de dispersão $(\mathrm{ZDW})$ da fibra. Isto implica que a dispersão cromática é pequena, e as quatro ondas estão espacialmente juntas. O acordo de fase, quando estabelecido em uma fibra com alta não linearidade, aumenta os efeitos do $4 \mathrm{WM}$.

Por simplificação e aplicação direta neste trabalho, vamos considerar apenas um bombeio com frequência $\omega_{1}$, o qual gera um ganho no sinal $\omega_{3}$ e $\omega_{4}$, e também no complementar $\omega_{3}^{\prime}$ e $\omega_{4}^{\prime 2}$. A partir da geração do sinal, múltiplas interações entre o bombeio e os sinais dão origem a termos secundários de 4WM, gerando novos campos. A figura abaixo exemplifica o surgimento de 4WM espúrios, devido à interação bombeio-sinal e sinal-sinal. A tabela 3.1 lista todos as possíveis combinações de 4WM que decorrem da interação do bombeio degenerado, do bombeio-sinal e

\footnotetext{
${ }^{1} \mathrm{Na}$ realidade, processos de segunda ordem podem ser observados em fibras devido a dipolos e quadrupolos magnéticamente induzidos, mas a eficiência de conversão é pequena tornando seu efeito desprezível.

${ }^{2} \mathrm{O}$ ganho na frequência do sinal é induzido injetando uma semente na fibra, isto é, um feixe de baixa intensidade.
} 


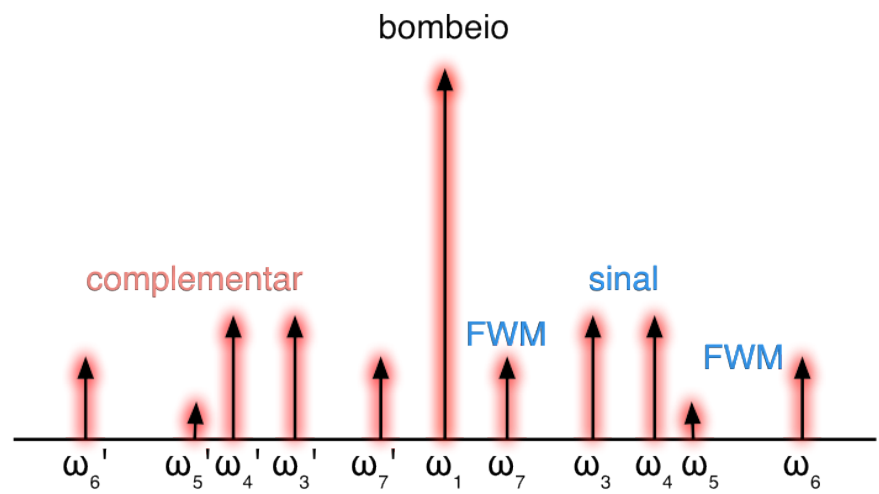

Figura 3.7: 4WM espúrios no bombeio degenerado: $\omega_{6}$ e $\omega_{7}$ são devidos à interação bombeiosinal e $\omega_{5}$ provém da interação sinal-sinal [35]. O complementar obedece a uma dinâmica semelhante.

do sinal-sinal. Os primeiros campos gerados pelo bombeio são denotados por $S_{1}$ e $S_{2}$ para o sinal, $I_{1}$ e $I_{2}$ para o complementar, e denominamos $P_{1}$ e $P_{2}$ os bombeios. A tabela expõe todos os 34 processos de $4 \mathrm{WM}$, ela contém 'x's", e indicam os termos envolvidos. Os processos podem ser degenerados, indicados com apenas dois " $x$ ", ou não degenerado, com três " $x$ ". O número total de termos envolvidos no processo de 4WM é igual ao número de "x's"na tabela.

Tanto para o bombeio degenerado quanto para o não degenerado, os 10 primeiros termos envolvem apenas os sinais e os complementares, e aparecem nos dois casos, somando um total de 24 termos. Para o bombeio degenerado, as linhas 12 e 13, e também 15 e 16 contabilizam o mesmo caso, portanto devem ser contadas apenas uma delas, contabilizando um total de $464 \mathrm{WM}$.

Para o bombeio não degenerado, até o processo 20 todos os termos devem ser contados, somando a estes, devemos incluir os processos relacionados com o segundo bombeio, de 20 a 30 . Há ainda quatro processos associados aos dois bombeios simultâneos. Portanto, 84 termos estão envolvidos nos processos de 4WM espúrios.

O processo de geração de novos feixes, envolve a interação de dois fótons no bombeio $\omega_{1}$ gerando novos fótons no sinal $\omega_{3}$, e complementar $\omega_{3}^{\prime}$. Como a interação dos pares de fótons é simultânea, correlações quânticas, como compressão de uma quadratura e emaranhamento entre os modos surgem do processo [52]. Os novos modos intensos gerados podem interagir entre si e entre os bombeios gerando outros modos correlacionados.

Abaixo do limiar de oscilação não há geração de campos intensos, mas ainda podem existir 
Tabela 3.1: Mistura de quatro ondas devido à interação entre os bombeios e o sinal.

\begin{tabular}{|c|c|c|c|c|c|c|}
\hline $4 \mathrm{WM}$ & $S_{1}$ & $S_{2}$ & $I_{1}$ & $I_{2}$ & $P_{1}$ & $P_{2}$ \\
\hline 1 & $\mathrm{x}$ & $\mathrm{x}$ & & & & \\
\hline 2 & & & $\mathrm{x}$ & $\mathrm{x}$ & & \\
\hline 3 & $\mathrm{x}$ & $\mathrm{x}$ & $\mathrm{x}$ & & & \\
\hline 4 & $\mathrm{x}$ & $\mathrm{x}$ & & $\mathrm{x}$ & & \\
\hline 5 & $\mathrm{x}$ & & $\mathrm{x}$ & $\mathrm{x}$ & & \\
\hline 6 & & $\mathrm{x}$ & $\mathrm{x}$ & $\mathrm{x}$ & & \\
\hline 7 & $\mathrm{x}$ & & $\mathrm{X}$ & & & \\
\hline 8 & & $\mathrm{x}$ & $\mathrm{x}$ & & & \\
\hline 9 & $\mathrm{x}$ & & & $\mathrm{x}$ & & \\
\hline 10 & & $\mathrm{x}$ & & $\mathrm{x}$ & & \\
\hline 11 & $\mathrm{x}$ & $\mathrm{x}$ & & & $\mathrm{x}$ & \\
\hline 12 & $\mathrm{x}$ & & & & $\mathrm{x}$ & \\
\hline 13 & & $\mathrm{x}$ & & & $\mathrm{x}$ & \\
\hline 14 & & & $\mathrm{X}$ & $\mathrm{x}$ & $\mathrm{x}$ & \\
\hline 15 & & & $\mathrm{x}$ & & $\mathrm{x}$ & \\
\hline 16 & & & & $\mathrm{x}$ & $\mathrm{x}$ & \\
\hline 17 & $\mathrm{x}$ & & $\mathrm{x}$ & & $\mathrm{x}$ & \\
\hline 18 & $\mathrm{x}$ & & & $\mathrm{x}$ & $\mathrm{x}$ & \\
\hline 19 & & $\mathrm{x}$ & $\mathrm{x}$ & & $\mathrm{x}$ & \\
\hline 20 & & $\mathrm{x}$ & & $\mathrm{x}$ & $\mathrm{x}$ & \\
\hline 21 & $\mathrm{x}$ & $\mathrm{x}$ & & & & $\mathrm{x}$ \\
\hline 22 & $\mathrm{X}$ & & & & & $\mathrm{x}$ \\
\hline 23 & & $\mathrm{x}$ & & & & $\mathrm{x}$ \\
\hline 24 & & & $\mathrm{X}$ & $\mathrm{X}$ & & $\mathrm{X}$ \\
\hline 25 & & & $\mathrm{x}$ & & & $\mathrm{X}$ \\
\hline 26 & & & & $\mathrm{x}$ & & $\mathrm{X}$ \\
\hline 27 & $\mathrm{x}$ & & $\mathrm{x}$ & & & $\mathrm{X}$ \\
\hline 28 & $\mathrm{x}$ & & & $\mathrm{x}$ & & $\mathrm{X}$ \\
\hline 29 & & $\mathrm{x}$ & $\mathrm{x}$ & & & $\mathrm{x}$ \\
\hline 30 & & $\mathrm{x}$ & & $\mathrm{x}$ & & $\mathrm{x}$ \\
\hline 31 & $\mathrm{x}$ & & & & $\mathrm{x}$ & $\mathrm{x}$ \\
\hline 32 & & $\mathrm{x}$ & & & $\mathrm{x}$ & $\mathrm{X}$ \\
\hline 33 & & & $\mathrm{X}$ & & $\mathrm{x}$ & $\mathrm{X}$ \\
\hline 34 & & & & $\mathrm{x}$ & $\mathrm{x}$ & $\mathrm{x}$ \\
\hline
\end{tabular}


correlações nos modos de vácuo [39]. Se estes modos forem gerados no interior de uma cavidade como a descrita na seção 3.3, os modos correlacionados estarão separados por múltiplas frequências do intervalo espectral livre. 



\section{Capítulo 4}

\section{Compressão em fibras}

Experimentos relacionados com a produção de estados comprimidos vêm sendo desenvolvidos desde 1985, e as ferramentas para a criação destes estados são bastante variadas, com a utilização de processos como: Efeito Kerr [32], 4WM [25] e geração de segundo harmônico [55]. O grupo da IBM Almaden Research Center, liderado por Shelby e Levenson, foi o primeiro a propor [27], e demonstrar experimentalmente [44], a geração de estados comprimidos em fibras ópticas através do processo de 4WM. A proposta era de realizar medidas de não demolição quântica do inglês (Quantum Nondemolition - QND) como forma de obter informações indiretamente em uma quadratura, de amplitude por exemplo, sem deturpar o estado do sistema. A técnica para tal medida foi correlacionar as quadraturas de amplitude e fase de dois campos intensos de bombeio por meio do $4 \mathrm{WM}$, e realizar medidas em apenas um dos campos, obtendo indiretamente informações da quadratura do outro campo sem alterar o estado do mesmo.

Nesta seção iremos descrever como o espectro de compressão nas quadraturas pode ser calculado quando o processo não linear responsável pelo efeito ocorre dentro de uma cavidade. Para uma cavidade perfeita, com poucas perdas e acoplamento crítico, o efeito será aumentar o grau de compressão nas quadraturas nas frequências de ressonância da cavidade. Iremos considerar o meio clássico, e desenvolver dois tratamentos, o primeiro no regime abaixo do limiar de oscilação, e o segundo, no regime acima do limiar.

\subsection{Tratamento estocástico}

O processo de mistura de quatro ondas não degenerado acopla o campo circulante em pares de frequências. Estas frequências estão relacionadas por $\omega_{3}+\omega_{4}=2 \omega_{1}$, onde $\omega_{3}$ é a frequência do 
campo denominado de sinal, $\omega_{4}$ do campo denominado de complementar e $\omega_{1}$ a frequencia do campo de bombeio. Descrevemos o tratamento quântico da interação como um par de fótons circulante do campo de bombeio interagindo dentro da cavidade. Fora da cavidade, existe um contínuo de frequências que somam $2 \omega_{1}$. Como o processo de interação ocorre dentro da cavidade, a eficiência do efeito causado pelo meio não linear sobre as bandas laterais é máximo nas frequências múltiplas do intervalo espectral livre. Portanto, o papel da cavidade aqui, é impor uma quantização na frequência dos efeitos não lineares.

Para um modo do campo eletromagnético fora da cavidade com frequência $\omega_{3}$, a frequência na cavidade será $\omega_{b}+m \omega_{c}$. Enquanto que, para um modo na frequência $\omega_{4}$, a frequência na cavidade será $\omega_{b}-m \omega_{c}$, onde $\omega_{c}$ é o intervalo espectral livre da cavidade. Na seção 3.2 deduzimos

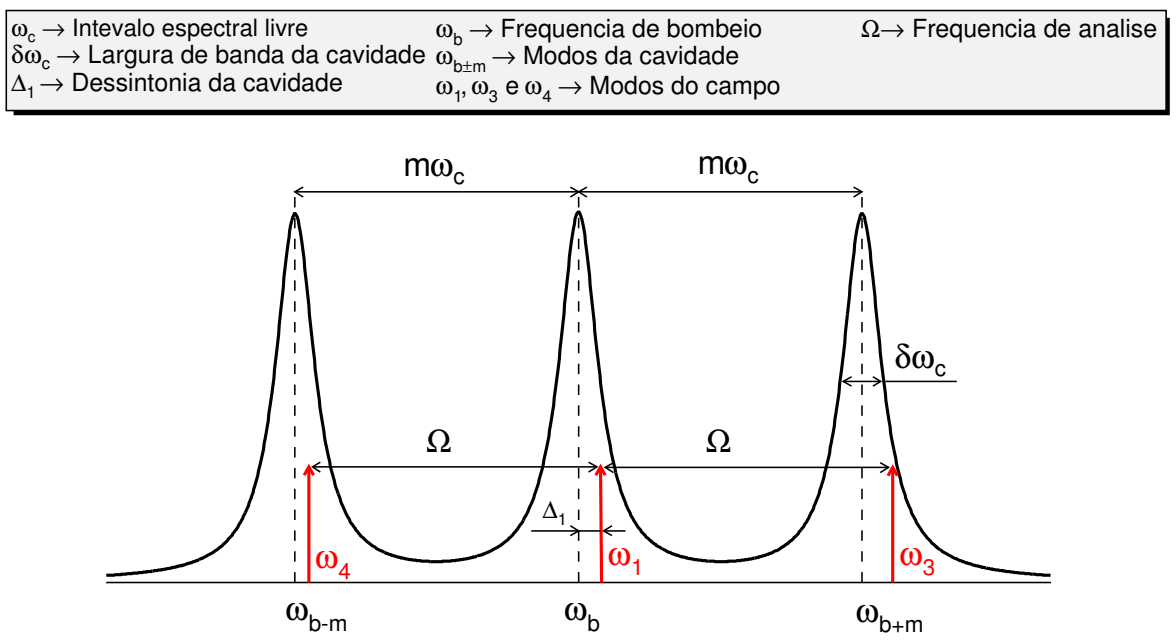

Figura 4.1: O intervalo espectral livre da cavidade é $\omega_{c}$, e é dado pela equação (3.3.8). Os modos de interesse são aqueles que estão distanciados em múltiplos inteiros de $\omega_{c}$. A frequência do campo de bombeio é representada por $\omega_{1}$, os campos de sinal e complementar são denominados de $\omega_{3}$ e $\omega_{4}$ respectivamente. A dessintonia dos campos com relação aos modos da cavidade é $\Delta_{i}$, com $i=1,3$ ou 4. A largura de linha de cada modo é $\delta \omega_{c}$, dado pela (3.3.9). A frequência de análise $\Omega$ é a frequência acessada pelo analisador de espectro.

a forma da Hamiltoniana não linear capaz de comprimir uma das quadraturas abaixo do limite quântico padrão (3.2.4), de maneira simplificada a escrevemos como

$$
\hat{H}_{4 W M}=i \hbar \epsilon \hat{a}_{1}^{2} \hat{a}_{3}^{\dagger} \hat{a}_{4}^{\dagger}+\text { h.c. }
$$


A constante complexa $\epsilon$ evidencia o acoplamento entre os diversos modos do campo intracavidade e compacta em seu valor o acordo de modo, o acordo de fase e as propriedades não lineares da fibra. Os modos dos campos de bombeio, sinal e complementar são designados pelos operadores de aniquilação da mecânica quântica $\hat{a}_{1}, \hat{a}_{3}$ e $\hat{a}_{4}$ respectivamente.

Fisicamente, a Hamiltoniana (4.1.1) representa um operador que descreve a conversão de dois fótons do bombeio com energia $\hbar \omega_{1}$ em dois fótons não degenerados dos campos de sinal e complementar, com energias $\hbar \omega_{3}$ e $\hbar \omega_{4}$. O processo inverso é descrito pelo Hermitiano conjugado da Hamiltoniana. Uma Hamiltoniana deste tipo foi utilizada inicialmente para descrever o processo de medida de não demolição quântica das quadraturas dos campos [21].

O acoplamento dos modos intracavidade com os feixes de bombeio, fora da cavidade, é descrita pela Hamiltoniana de acoplamento. Os feixes de bombeio são intensos e portanto são tratados classicamente

$$
\hat{H}_{e x t}=i \hbar\left[E_{1} \hat{a}_{1}^{\dagger} \exp \left(-i 2 \omega_{1} t\right)+E_{3} \hat{a}_{3}^{\dagger} \exp \left(-i \omega_{3} t\right)+E_{4} \hat{a}_{4}^{\dagger} \exp \left(-i \omega_{4} t\right)\right]+\text { h.c. }
$$

O caráter do tratamento clássico dos campos é simbolizado nas representações destes modos pelas grandezas complexas $E_{1}, E_{3}$ e $E_{4}$, que são proporcionais às amplitudes dos campos clássicos de entrada. É importante notar também que consideramos a frequência do campo de bombeio de $2 \omega_{1}$, descrito no argumento da exponencial. Esta é uma forma de ilustrar a interação de dois fótons do campo de bombeio no processo.

No caso de uma cavidade vazia, sem um meio sólido interagindo com o campo, como ocorre em uma cavidade Fabry-Perot por exemplo, a Hamiltoniana que descreve os modos dos campos intracavidade é $\hat{H}_{\text {livre }}=\sum_{1,3,4} \hbar \omega_{i} \hat{a}_{i}^{\dagger} \hat{a}_{i}$, ou seja, o campo é descrito como um oscilador harmônico no estado fundamental. Como visto na seção 3.4, a ação da cavidade sobre os campos é estabelecer um meio de interação entre os modos internos e o banho térmico, entendido como flutuações do vácuo. O acoplamento da onda eletromagnética aprisionada na cavidade com as flutuações de vácuo não é o único meio pelo qual o campo sofre atenuação, outras formas de interação como: o acoplamento dos modos internos com as oscilações elásticas do meio material, a absorção de fótons pelos átomos da fibra e outras formas relacionadas com a estrutura da cavidade, são responsáveis pela atenuação. Uma forma conveniente de modelar estas perdas é através do acoplamento dos modos internos com o meio exterior, estimado como responsável por toda atenuação sofrida pelo campo. Este modo externo é descrito pelo operador $\hat{\Gamma}_{i}$, que representa o operador de aniquilação do banho

$$
\hat{H}_{b a n h o}=\hbar \sum_{i=b, s, c} \hat{a}_{i} \hat{\Gamma}_{i}^{\dagger}+\text { h.c. }
$$


Dessa forma, escrevemos a Hamiltoniana que descreve o processo geral de mistura de quatro ondas dentro de uma cavidade como a soma destas quatro Hamiltonianas [11]

$$
\hat{H}=\hat{H}_{\text {livre }}+\hat{H}_{e x t}+\hat{H}_{4 W M}+\hat{H}_{\text {banho }}
$$

que contém o efeito da interação da radiação do campo com o meio não linear, assim como a interação dos modos intracavidade com o campo de bombeio, e também a ação do banho térmico, modelado como flutuações de vácuo, sobre os campos internos.

As propriedades estatísticas do sistema são descritas pelo operador densidade $\hat{\rho}$ que satisfaz a equação de movimento

$$
\frac{\partial \hat{\rho}}{\partial t}=\frac{1}{i \hbar}[\hat{H}, \hat{\rho}],
$$

onde $\operatorname{Tr}_{B, S} \hat{\rho}(t)=1$, e onde todos os operadores estão na representação de Schrödinger, e o traço é tomado sobre o banho e o sistema.

Em geral estamos interessados apenas nas propriedades estatísticas do sistema. Isto é, se desejamos conhecer alguma variável do sistema $M$, estamos interessados em conhecer o valor de $\langle M(t)\rangle=\operatorname{Tr}_{B, S} \hat{M} \hat{\rho}(t)=\operatorname{Tr}_{S} \hat{M} \operatorname{Tr}_{B} \hat{\rho}(t)$, onde o traço é tomado sobre o sistema e o banho, e os operadores destes comutam com os operadores do sistema, e $\hat{M}$ contém apenas operadores do sistema. Portanto, gostaríamos de obter valores esperados de variáveis do sistema sem a necessidade de conhecer o estado do reservatório. Isto é feito definindo o operador densidade reduzido, tomando o traço sobre o operador densidade, que contém apenas operadores do sistema. É interessante então, remover as informações desnecessárias da (4.1.5), e obter a equação de movimento diretamente em termos do operador densidade do sistema.

Este procedimento é feito passando a equação (4.1.5) para a representação de interação, eliminando os termos que variam rapidamente no sistema. Como resultado deste processo, obtemos a equação de dinâmica para o operador densidade na representação de interação, envolvendo apenas a Hamiltoniana de interação dos modos do campo com os operadores do banho. Neste processo, lançamos a dependência temporal sobre os operadores do sistema, descritos como uma evolução temporal em função da atuação das outras Hamiltonianas que não a de interação.

Dificilmente este procedimento nos leva a uma solução exata do sistema, e tratamos o problema no esboço da teoria de perturbação [10]. No processo, assume-se que a Hamiltoniana de interação do sistema com o banho possa ser escrita como uma soma de produtos dos operadores do campo com os operadores do banho, na representação de Schrödinger (4.1.3).

A diferença $\hat{\rho}(t)-\hat{\rho}\left(t_{0}\right)$, representa a evolução do sistema transcorrido um intervalo de tempo $t-t_{0}$. Por definição, um sistema é dito Markoviano quando seu futuro é determinado apenas pelo presente, sem dependência com o passado. Este é um sistema sem memória, cuja evolução é ditada pelo presente. Isso parece ser o suficiente para garantir que a energia que flui para o 
reservatório não irá retornar para o sistema. Caso contrário, o sistema desenvolveria memória [30].

Os operadores do banho possuem um tempo finito de correlação. Tipicamente eles são não nulos em um intervalo de tempo $\tau_{B}$, que é chamado de tempo de correlação do reservatório. Desta forma, se quisermos eliminar a influência do reservatório sobre o sistema, devemos estudar a evolução do sistema para tempos bem maiores do que o tempo de correlação do reservatório. Como o tempo de correlação é indiretamente proporcional à largura de linha, em termos desta escala, concluímos que a largura de linha do reservatório deve ser bem maior que a largura de linha do sistema. Superiormente o tempo de análise é limitado pelo amortecimento do sistema. $\mathrm{Na}$ representação de interação, com a aproximação de Markov o tempo de correlação do reservatório tende a zero. Esta aproximação permite a eliminação dos operadores do banho na (4.1.5), levando a uma equação de movimento que possui apenas operadores do sistema. Este tratamento é conhecido como representação de Schrödinger-Markov, e a equação resultante denominada de equação mestra [55, 15, 30].

Para o reservatório em equilíbrio térmico e com temperatura $T=0$, podemos escrever a equação mestra como

$$
\frac{\partial \hat{\rho}}{\partial t}=\frac{1}{i \hbar}\left[\hat{H}_{\text {livre }}+\hat{H}_{e x t}+\hat{H}_{4 W M}, \hat{\rho}\right]+\sum_{\imath=b, s, c} \hat{L}_{\imath} \hat{\rho} .
$$

O processo de obtenção da equação mestra elimina os operadores do banho, mas sua influência ainda existe, e é manifestada em termos dos operadores do sistema, representada nos operadores de Lindblad

$$
\hat{L}_{\imath}=\gamma_{\imath}\left(2 \hat{a}_{\imath} \hat{\rho} \hat{a}_{\imath}^{\dagger}-\hat{a}_{\imath}^{\dagger} \hat{a}_{\imath} \hat{\rho}-\hat{\rho} \hat{a}_{\imath}^{\dagger} \hat{a}_{\imath}\right),
$$

onde $\gamma_{\imath}$ está relacionado com o amortecimento do sistema, devido ao acoplador e às perdas espúrias.

Em geral, a equação de dinâmica para as variáveis que estão diretamente ligadas com as informações do sistema, como a amplitude média do estado ou o número de fótons, são dificilmente obtidas pela (4.1.6). Para lidar com esta situação, é conveniente converter a equação mestra em termos de operadores para uma equação equivalente, em termos de números complexos c-number - e derivadas parciais. Existem diversas abordagens nas quais isso pode ser feito, e que levam a diferentes representações para o mesmo problema. Neste trabalho escolhemos a representação de Wigner, escolha baseada na exposição direta das propriedades físicas do problema.

Na próxima seção faremos uma exposição breve de como a passagem de uma equação diferencial parcial envolvendo operadores, para uma equação diferencial parcial envolvendo números complexos, pode ser feita através da função de Wigner, já definida na seção 2.3. 


\subsection{Representação de Wigner}

A conversão da equação mestra (4.1.6) escrita em termos de operadores da mecânica quântica em uma equação diferencial parcial escrita em termos de c-numbers, pode ser feita através da definição da função característica [55]

$$
\chi(\beta)=\operatorname{Tr}(\hat{D} \hat{\rho})
$$

onde $\hat{D}$ é o operador deslocamento (2.3.7). E a função de Wigner pode ser definida em termos da função característica como

$$
W(\alpha)=\int \exp \left(\beta^{*} \alpha+\beta \alpha^{*}\right) \chi(\beta) d^{2} \beta
$$

A equação de movimento para $\chi(\beta)$ é dada por

$$
\frac{\partial \chi(\beta)}{\partial t}=\operatorname{Tr}\left\{\hat{D} \frac{\partial \hat{\rho}}{\partial t}\right\}
$$

A partir da fórmula de Baker-Hausdorff podemos escrever o operador deslocamento em ordem normal e obter as seguintes relações de equivalência entre operadores e derivadas:

$$
\hat{a}^{\dagger} \hat{D}=\left(\frac{\partial}{\partial \beta}+\frac{\beta^{*}}{2}\right) \hat{D}, \quad \text { e } \quad \hat{D} \hat{a}=\left(\frac{-\beta}{2}-\frac{\partial}{\partial \beta^{*}}\right) \hat{D} .
$$

Da mesma forma, escrevendo o operador deslocamento em ordem antinormal obtemos:

$$
\hat{D} \hat{a}^{\dagger}=\left(\frac{\partial}{\partial \beta}-\frac{\beta^{*}}{2}\right) \hat{D}, \quad \text { e } \hat{a} \hat{D}=\left(\frac{\beta}{2}-\frac{\partial}{\partial \beta^{*}}\right) \hat{D} .
$$

Então, com estas relações de equivalência e as equações (4.2.1) e (4.2.3), podemos escrever a equação mestra (4.1.6) para a função característica. Tomando a transformada de Fourier desta equação, obtemos a equação de dinâmica para a função de Wigner com origem na equação mestra. A conclusão final deste procedimento é que podemos substituir o operador densidade da equação mestra pela função de Wigner, e a atuação dos operadores sobre o operador densidade é substituída por operações de derivadas e multiplicações por números complexos sobre a função 
de Wigner. As relações de equivalência estão expostas abaixo:

$$
\begin{array}{ll}
\hat{a} \hat{\rho} \rightarrow\left(\alpha+\frac{1}{2} \frac{\partial}{\partial \alpha^{*}}\right) W\left(\alpha, \alpha^{*}\right), & \hat{a}^{\dagger} \hat{\rho} \rightarrow\left(\alpha^{*}-\frac{1}{2} \frac{\partial}{\partial \alpha}\right) W\left(\alpha, \alpha^{*}\right), \\
\hat{\rho} \hat{a} \rightarrow\left(\alpha-\frac{1}{2} \frac{\partial}{\partial \alpha^{*}}\right) W\left(\alpha, \alpha^{*}\right), & \hat{\rho} \hat{a}^{\dagger} \rightarrow\left(\alpha^{*}+\frac{1}{2} \frac{\partial}{\partial \alpha}\right) W\left(\alpha, \alpha^{*}\right) .
\end{array}
$$

\subsection{Dinâmica do $4 \mathrm{WM}$}

O tratamento teórico do processo de mistura de quatro ondas em fibras inicia-se escrevendo a equação mestra (4.1.6) para o operador densidade. De acordo com a seção 4.2, podemos interpretar a atuação dos operadores da mecânica quântica sobre o operador densidade como operações de derivada e multiplicação atuando sobre a função de Wigner

$$
\begin{aligned}
& \frac{\partial W\left(\boldsymbol{\alpha}, \boldsymbol{\alpha}^{*}\right)}{\partial t}=\left\{-\frac{\partial}{\partial \alpha_{1}}\left[\left(-i 2 \omega_{1}-\gamma_{1}\right) \alpha_{1}+E_{b} \exp \left(-i 2 \omega_{b} t\right)-2 \epsilon^{*} \alpha_{1}^{*} \alpha_{3} \alpha_{4}\right]\right. \\
& -\frac{\partial}{\partial \alpha_{1}^{*}}\left[\left(i 2 \omega_{1}-\gamma_{1}\right) \alpha_{1}^{*}+E_{b}^{*} \exp \left(i 2 \omega_{b} t\right)-2 \epsilon \alpha_{1} \alpha_{3}^{*} \alpha_{4}^{*}\right] \\
& -\frac{\partial}{\partial \alpha_{3}}\left[\left(-i \omega_{3}-\gamma_{3}\right) \alpha_{3}+E_{s} \exp \left(-i \omega_{s} t\right)+\epsilon \alpha_{1}^{2} \alpha_{4}^{*}\right] \\
& -\frac{\partial}{\partial \alpha_{3}^{*}}\left[\left(i \omega_{3}-\gamma_{3}\right) \alpha_{3}^{*}+E_{s}^{*} \exp \left(i \omega_{s} t\right)+\epsilon^{*} \alpha_{1}^{* 2} \alpha_{4}\right] \\
& -\frac{\partial}{\partial \alpha_{4}}\left[\left(-i \omega_{4}-\gamma_{4}\right) \alpha_{4}+E_{c} \exp \left(-i \omega_{c} t\right)+\epsilon \alpha_{1}^{2} \alpha_{3}^{*}\right] \\
& -\frac{\partial}{\partial \alpha_{4}^{*}}\left[\left(i \omega_{4}-\gamma_{4}\right) \alpha_{4}^{*}+E_{c}^{*} \exp \left(i \omega_{c} t\right)+\epsilon^{*} \alpha_{1}^{* 2} \alpha_{3}\right] \\
& +\frac{1}{2}\left(2 \gamma_{1} \frac{\partial^{2}}{\partial \alpha_{1} \alpha_{1}^{*}}+2 \gamma_{3} \frac{\partial^{2}}{\partial \alpha_{3} \alpha_{3}^{*}}+2 \gamma_{4} \frac{\partial^{2}}{\partial \alpha_{4} \alpha_{4}^{*}}\right) \\
& +\epsilon\left(\frac{1}{2} \alpha_{1} \frac{\partial^{3}}{\partial \alpha_{1}^{*} \alpha_{3} \alpha_{4}}-\frac{1}{4} \alpha_{4}^{*} \frac{\partial^{3}}{\partial \alpha_{1}^{*} \alpha_{1}^{*} \alpha_{3}}-\frac{1}{4} \alpha_{3}^{*} \frac{\partial^{3}}{\partial \alpha_{1}^{*} \alpha_{1}^{*} \alpha_{4}}\right) \\
& \left.+\epsilon^{*}\left(\frac{1}{2} \alpha_{1}^{*} \frac{\partial^{3}}{\partial \alpha_{1} \alpha_{3}^{*} \alpha_{4}^{*}}-\frac{1}{4} \alpha_{4} \frac{\partial^{3}}{\partial \alpha_{1} \alpha_{1} \alpha_{3}^{*}}-\frac{1}{4} \alpha_{3} \frac{\partial^{3}}{\partial \alpha_{1} \alpha_{1} \alpha_{4}^{*}}\right)\right\} W\left(\boldsymbol{\alpha}, \boldsymbol{\alpha}^{*}\right),
\end{aligned}
$$

onde $\boldsymbol{\alpha}$ é um vetor representativo das grandezas complexas $\left\{\alpha_{1}, \alpha_{1}^{*}, \alpha_{3}, \alpha_{3}^{*}, \alpha_{4}, \alpha_{4}^{*}\right\}$. Aqui, a constante $\gamma_{i}$ representa o acoplamento do modo $i$ do campo propagante na cavidade com o meio externo.

A expressão acima descreve a dinâmica mais geral dos modos dos campos quânticos quando sujeitos ao processo não linear de mistura de quatro ondas que ocorre em uma fibra de alta não linearidade geometricamente estruturada na forma de uma cavidade. Algumas simplificações 
são aplicáveis e facilmente justificáveis em termos das propriedades físicas do sistema. A parte mais delicada da dinâmica, e que impossibilita o tratamento analítico do problema, é causada pelas duas últimas linhas da equação, as derivadas terceiras destes termos tornam o problema analiticamente complicado. Sem nenhuma justificativa mais rigorosa, iremos impor a anulação destes termos tomando como base o argumento de que estes são menos significativos no processo de interação dos campos com o meio do que os termos lineares de modos distintos [37].

Sem os termos de derivada terceira, a equação (4.3.1) é reconhecida como uma equação de Fokker-Planck. Esta equação foi inicialmente aplicada como uma análise alternativa do estudo do movimento browniano, descrito como uma equação de Langevin. Planck derivou a equação que leva seu nome como uma aproximação da equação mestra [23]. Fisicamente, devemos restringir o processo de evolução do sistema estocástico a pequenos "pulos", isto é, o sistema evolui de uma forma não brusca, com pequenas mudanças a cada interação. Isto é o mesmo que considerar um processo do tipo random walks, onde o "tamanho" de cada passo é considerado pequeno, mas o número de passos é grande o suficiente para que o deslocamento médio resultante possa ser tratado de forma contínua.

Random walks é um formalismo matemático que estuda o movimento de um sistema como um conjunto de passos aleatórios, considerados sobre a aproximação de Markov. Este formalismo é comum em muitas áreas de estudos, como na física, economia, biologia, estatística de população, etc. A maneira mais interessante de descrever um processo deste tipo é através de seu tratamento diferencial, no qual expressamos, fundamentado na teoria de probabilidades, a probabilidade de encontrar uma partícula ou o estado de um sistema, que possa ser tratado no âmbito de variáveis aleatórias, na posição $x$ ou num estado específico, como uma equação diferencial [14]

$$
\frac{\partial}{\partial t} P(\mathbf{x}, t)=-\sum_{\imath} \frac{\partial}{\partial x_{\imath}} A_{\imath}(\mathbf{x}, t)+\frac{1}{2} \sum_{\imath \jmath} \frac{\partial}{\partial x_{\imath}} \frac{\partial}{\partial x_{\jmath}}\left[\mathbb{B}(\mathbf{x}, t) \mathbb{B}^{\top}(\mathbf{x}, t)\right]_{\imath \jmath} P(\mathbf{x}, t) .
$$

Esta equação é conhecida como equação de Fokker-Planck, ou equação de difusão. O termo pertencente à primeira derivada é responsável pelos valores médios determinísticos das variáveis, e é conhecido como termo de arrasto. O termo englobado na segunda derivada é responsável pelo "alargamento", difusão dos valores médios, e é conhecido como termo de difusão. É interessante a conexão direta entre a dinâmica encontrada para o processo de interação não linear, entre os fótons dos campos e os átomos que compõem o meio material, e processos tratados como random walks, como o movimento browniano.

A equação (4.3.2) pode ser escrita em uma forma completamente equivalente

$$
\frac{d \mathbf{x}}{d t}=\mathbf{A}(\mathbf{x}, t)+\mathbb{B}(\mathbf{x}) \boldsymbol{\eta}(t)
$$


onde $\boldsymbol{\eta}(t)$ são forças estocásticas com média zero e $\delta$ correlacionadas no tempo [55]

$$
\begin{aligned}
& \left\langle\eta_{\imath}(t)\right\rangle=\left\langle\eta_{\imath}^{\dagger}(t)\right\rangle=0, \\
& \left\langle\eta_{\imath}(t) \eta_{\jmath}^{\dagger}\left(t^{\prime}\right)\right\rangle=\delta_{\imath \jmath} \delta\left(t-t^{\prime}\right)
\end{aligned}
$$

A equação (4.3.3) é conhecida como equação de Langevin. A passagem da equação de FokkerPlanck para a forma da equação de Langevin pode ser derivada em termos das equação diferenciais estocásticas de Ito [16].

Desta forma, comparando a equação mestra (4.3.1) encontrada para o sistema, com a equação de Fokker-Planck (4.3.2), podemos escrever as equações de dinâmica dos modos na forma da equação de Langevin, com o vetor de arrasto dado por

$$
\mathbb{A}=\left[\begin{array}{c}
-\gamma_{1}\left(1-i \Delta_{1}\right) \alpha_{1}+E_{b}-2 \epsilon^{*} \alpha_{1}^{*} \alpha_{3} \alpha_{4} \\
-\gamma_{1}\left(1+i \Delta_{1}\right) \alpha_{1}^{*}+E_{b}^{*}-2 \epsilon \alpha_{1} \alpha_{3}^{*} \alpha_{4}^{*} \\
-\gamma_{3}\left(1-i \Delta_{3}\right) \alpha_{3}+E_{s}+\epsilon \alpha_{1}^{2} \alpha_{4}^{*} \\
-\gamma_{3}\left(1+i \Delta_{3}\right) \alpha_{3}^{*}+E_{s}^{*}+\epsilon^{*} \alpha_{1}^{* 2} \alpha_{4} \\
-\gamma_{4}\left(1-i \Delta_{4}\right) \alpha_{4}+E_{c}+\epsilon \alpha_{1}^{2} \alpha_{3}^{*} \\
-\gamma_{4}\left(1+i \Delta_{4}\right) \alpha_{4}^{*}+E_{c}^{*}+\epsilon^{*} \alpha_{1}^{* 2} \alpha_{3}
\end{array}\right]
$$

$$
\mathbb{B}=\left[\begin{array}{cccccc}
i \sqrt{\gamma_{1} / 2} & \sqrt{\gamma_{1} / 2} & 0 & 0 & 0 & 0 \\
-i \sqrt{\gamma_{1} / 2} & \sqrt{\gamma_{1} / 2} & 0 & 0 & 0 & 0 \\
0 & 0 & i \sqrt{\gamma_{3} / 2} & \sqrt{\gamma_{3} / 2} & 0 & 0 \\
0 & 0 & -i \sqrt{\gamma_{3} / 2} & \sqrt{\gamma_{3} / 2} & 0 & 0 \\
0 & 0 & 0 & 0 & i \sqrt{\gamma_{4} / 2} & \sqrt{\gamma_{4} / 2} \\
0 & 0 & 0 & 0 & -i \sqrt{\gamma_{4} / 2} & \sqrt{\gamma_{4} / 2}
\end{array}\right]
$$

A aproximação de onda girante foi feita, levando as grandezas complexas dos modos para um referencial girante na frequência dos campos de bombeio, $\alpha_{\imath} \rightarrow \alpha_{\imath} \exp \left(-i \omega_{\jmath}\right)$, com $\imath=1,3,4$ e $\jmath=b, s, c$ respectivamente. Os $\Delta^{\prime} s$ são as dessintonias normalizadas pela largura de linha dos modos da cavidade, $\Delta_{1}=\left(\omega_{b}-2 \omega_{1}\right) / \gamma_{1}, \Delta_{3}=\left(\omega_{s}-\omega_{3}\right) / \gamma_{3}$ e $\Delta_{4}=\left(\omega_{c}-\omega_{4}\right) / \gamma_{4}$. Note que a matriz de difusão, definida como $\mathbb{B} \cdot \mathbb{B}^{\top}$, depende apenas do acoplamento da cavidade com o ambiente. Ela está diretamente ligada às variâncias dos modos.

A equação de Langevin define a distribuição de probabilidade de Wigner como um conjunto complexo de equações diferenciais estocásticas no espaço de fase de seis dimensões. Estas seis equações estão acopladas entre si e não é possível resolvê-las independentemente. A atuação do vácuo é responsável pela existência de forças estocásticas, gerando um processo difusivo nos valores médios dos campos. A não consideração destas forças recupera a dinâmica do sistema 
isolado, equação (4.1.5).

A atuação do reservatório sobre o sistema com perdas é responsável por adicionar forças randômicas, tendo como consequência o acréscimo de ruídos ao sistema. Caso o interior do sistema seja caracterizado por possuir uma estatística específica de flutuação, e as flutuações do reservatório sejam caracterizadas por uma estatística diferente, as flutuações do reservatório irão forçar o sistema a ter a mesma estatística do reservatório. As forças estocásticas introduzidas pela equação de Langevin substituem como um todo a atuação do reservatório sobre o sistema. Estas forças têm um papel fundamental no entendimento da dinâmica do sistema fundamentado na mecânica quântica, pois representam termos de atenuação que mantém as relações de comutação válidas.

É importante neste ponto fazer algumas considerações a respeito do sistema. A equação (4.3.3) com o vetor de arrasto dado por (4.5.4), e matriz de difusão definida acima, descrevem as dinâmicas dos modos dos campos intracavidade, interagindo entre si pelo processo não linear de mistura de quatro ondas que ocorre em fibras com alta não linearidade, sobre a influência do vácuo. Para um sistema insensível a distâncias curtas em comprimento de onda, é normal considerar a mesma influência do reservatório para todos os modos dos campos intracavidade. Esta aproximação é de fato válida dentro de alguns intervalos espectrais livres quando o acoplador, que faz a porta de comunicação dos modos internos como os modos externos, for insensível a diferentes comprimentos de onda, e a frequência do campo de bombeio for próximo ao zero de dispersão da fibra.

Para um campo de bombeio monocromático, a interação dos modos internos por meio do acoplador é estabelecido apenas para um modo, $\alpha_{1}$. Como veremos adiante, a implicação da não existência de um campo estimulante no outros modos da cavidade resulta no equilíbrio das intensidades dos modos $\alpha_{3}$ e $\alpha_{4}$. 


\subsection{Solução estacionária}

A solução estacionária é obtida através das equações de dinâmica (4.3.3), fazendo as derivadas temporais nulas e desprezando as forças estocásticas

$$
\begin{gathered}
0=-\gamma_{1}\left(1-i \Delta_{1}\right) \alpha_{1}+E-2 \epsilon^{*} \alpha_{1}^{*} \alpha_{3} \alpha_{4}, \\
0=-\gamma_{1}\left(1+i \Delta_{1}\right) \alpha_{1}^{*}+E^{*}-2 \epsilon \alpha_{1} \alpha_{3}^{*} \alpha_{4}^{*}, \\
0=-\gamma_{3}\left(1-i \Delta_{3}\right) \alpha_{3}+\epsilon \alpha_{1}^{2} \alpha_{4}^{*}, \\
0=-\gamma_{3}\left(1+i \Delta_{3}\right) \alpha_{3}^{*}+\epsilon^{*} \alpha_{1}^{* 2} \alpha_{4}, \\
0=-\gamma_{4}\left(1-i \Delta_{4}\right) \alpha_{4}+\epsilon \alpha_{1}^{2} \alpha_{3}^{*}, \\
0=-\gamma_{4}\left(1+i \Delta_{4}\right) \alpha_{4}^{*}+\epsilon^{*} \alpha_{1}^{* 2} \alpha_{3} .
\end{gathered}
$$

No conjunto de equações acima consideramos a injeção do campo apenas no modo $\alpha_{1}$, e eliminamos o índice $b$. Escrevendo $\alpha_{\imath}=\left|\alpha_{\imath}\right| \exp \left(i \phi_{\imath}\right)$, temos que $\alpha_{\imath} \alpha_{\imath}^{*}=\left|\alpha_{\imath}\right|^{2} \equiv P_{\imath}$, representa a potência $\imath$ do modo circulante na cavidade. Multiplicando a equação (4.4.3) pela sua complexa conjugada (4.4.4), e também a (4.4.5) pela (4.4.6), temos

$$
\begin{aligned}
& |\epsilon|^{2} P_{1}^{2} P_{4}=\gamma_{3}^{2}\left(1+\Delta_{3}^{2}\right) P_{3}, \\
& |\epsilon|^{2} P_{1}^{2} P_{3}=\gamma_{4}^{2}\left(1+\Delta_{4}^{2}\right) P_{4} .
\end{aligned}
$$

As equações para as potências dos campos gerados (4.4.7) e (4.4.8) são semelhantes, indicando uma mesma dinâmica para o número de fótons, sendo ditadas pela resposta da cavidade a estes modos e às dessintonias da mesma. Vemos as diferentes respostas, dividindo uma pela outra

$$
\frac{P_{4}}{P_{3}}=\frac{\gamma_{3}}{\gamma_{4}} \sqrt{\frac{1+\Delta_{3}^{2}}{1+\Delta_{4}^{2}}} .
$$

Quando ainda não há geração de campos, é possível relacionar pela (4.4.1) a potência do modo do campo intracavidade $\alpha_{1}$ com a potência do campo de injeção $E$

$$
P_{1, \text { abaixo }}=\frac{|E|^{2}}{\gamma_{1}^{2}\left(1+\Delta_{1}^{2}\right)}
$$

Considerando que a cavidade possua a mesma resposta para ambos os campos gerados $\gamma_{3}=$ $\gamma_{4}=\gamma$, e que as dessintonias sejam as mesma $\Delta_{3}=\Delta_{4}=\Delta$, obtemos a potência do modo 
intracavidade do campo de bombeio após a geração dos campos

$$
P_{1}=\frac{\gamma \sqrt{1+\Delta^{2}}}{|\epsilon|}
$$

Notamos que o campo de bombeio intracavidade é limitado superiormente por um valor fixo, sendo inversamente proporcional ao fator de não linearidade da fibra. O valor máximo da intensidade é atingido no momento em que se inicia a geração dos feixes gêmeos. A existência do limiar de oscilação em OPO (Optical Parametric Oscillator), para a geração de feixes em cavidade do tipo Fabry-Perot, com um cristal altamente não linear já foi verificado em [13, 36]. Definimos a potência mínima como a menor potência necessária para iniciar a geração de feixes gêmeos

$$
P_{1, \min }=\frac{\gamma}{\epsilon}
$$

revelando que o limiar de oscilação para a geração de feixes gêmeos é diminuído de duas formas, aumentado a finesse da cavidade ou o fator de não lineardidade da fibra.

Nesta condição, a equação (4.4.3) fornece a relação de fase entre os três campos

$$
\exp \left[i\left(\phi_{+}-2 \phi_{1}\right)\right]=\frac{\epsilon P_{1}}{\gamma(1-i \Delta)}
$$

onde $\phi_{+}=\phi_{3}+\phi_{4}$ é a soma das fases dos campos gerados. Inserindo a equação (4.4.11) na (4.4.13) e considerando a constante $\epsilon$ real

$$
\exp \left[i\left(\phi_{+}-2 \phi_{1}\right)\right]=\frac{\sqrt{1+\Delta^{2}}}{1-i \Delta}
$$

obtemos que há uma relação de fase fixa entre os três campos, sendo determinada apenas pela dessintonia. Mantendo a fase do campo de bombeio fixa, temos um controle da fase relativa entre os modos do sinal e complementar pela dessintonia da cavidade nestes modos. Acima do limiar, segue das (4.4.2), (4.4.10), (4.4.11) e da (4.4.14) que

$$
P=\frac{\gamma_{1}}{2 \epsilon}\left[-\frac{1-\Delta \Delta_{1}}{\sqrt{1+\Delta^{2}}} \pm \sqrt{\frac{\left(1-\Delta \Delta_{1}\right)^{2}}{1+\Delta^{2}}-\left(1+\Delta_{1}^{2}\right)(1-\sigma)}\right]
$$

A relação acima informa a potência dos campos de sinal ou complementar em função da variável $\sigma$, definida como a intensidade do campo de bombeio intracavidade em unidades do limiar de oscilação. No caso em que o produto das dessintonias $\Delta \Delta_{1}$ é menor do que um, apenas a solução representada pela soma dos dois termos entre colchetes é aceitável. Em contrapartida, caso o produto das dessintonias seja maior do que um, as intensidades dos campos gerados admitem 
duas soluções, situação descrita como biestabilidade, estudado em [43, 13]. Notamos na figura
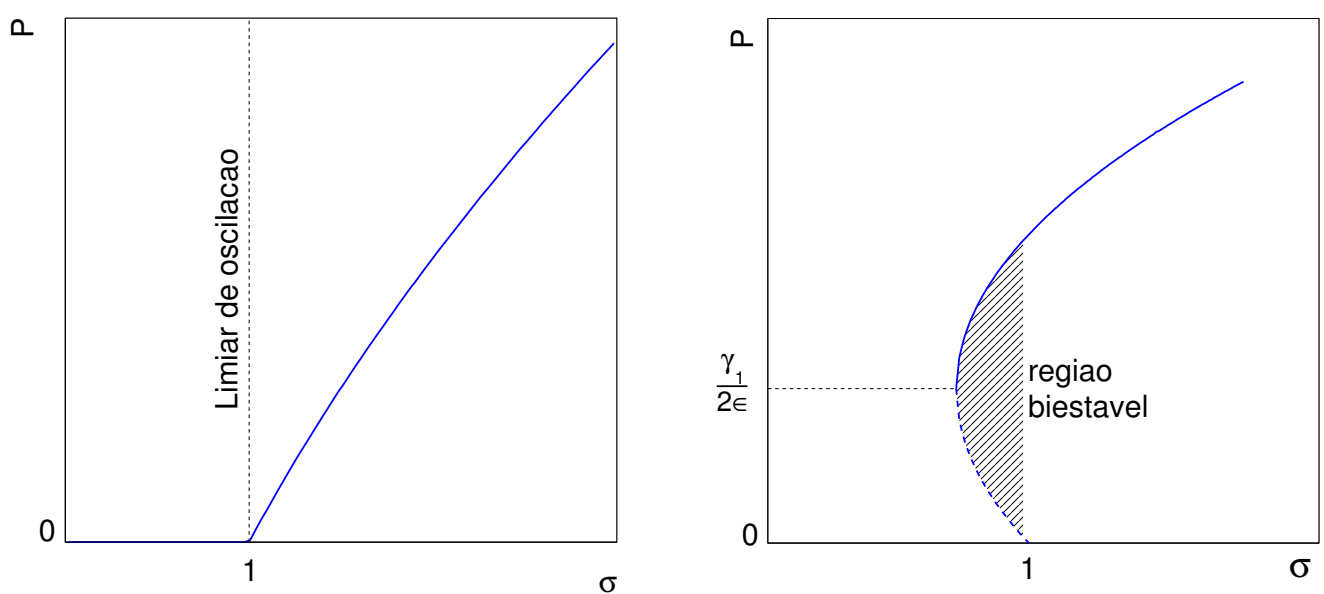

Figura 4.2: Possíveis soluções estacionárias para as intensidades dos modos internos dos campos gerados na cavidade. Antes do limiar, $\sigma<1$, não há feixes gêmeos e o modo interno do campo de bombeio escala linearmente com o campo clássico injetado. O primeiro gráfico mostra a solução única para o caso de dessintonias nulas. O segundo gráfico mostras as duas possíveis soluções para dessintonias satisfazendo $\Delta_{1}=\Delta$ e $\Delta_{1} \Delta=3$.

(4.2), que uma vez atingido o limiar de oscilação para dessintonias condicionadas à $\Delta_{1} \Delta>1$, é possível haver feixes gêmeos com intensidade menores àquela do limiar. Apesar da capacidade de manipulação dos estados dos campos nestas duas regiões de biestabilidade, isto é, de passar de uma região à outra de uma forma contínua e controlável, estas são regiões de equilíbrio delicado, perturbável por problemas técnicos.

\subsection{Equações linearizadas}

Nesta seção, nos concentraremos mais nas flutuações dos campos ao invés de seus valores médios. Para isso, consideramos a descrição das grandezas complexas representativa dos modos intracavidade como seus valores médios mais um termo de flutuação

$$
\alpha_{\imath}=\bar{\alpha}_{\imath}+\delta \alpha_{\imath}
$$

onde $\bar{\alpha}_{\imath}$ são os valores médios dos modos, cujas soluções foram obtidas na seção anterior para as quantidades $\left|\bar{\alpha}_{\imath}\right|^{2}$, com $\imath=1,3$ e 4 . O estudo das propriedades dos modos pelo processo de linearização foi primeiramente estudado em [31]. O processo consiste em reescrever as equações 
de Langevin (4.3.3), substituindo as grandezas complexas por (4.5.1), e desprezando os termos quadráticos e de ordem superior em $\delta$. As equações linearizadas podem ser escritas segundo a equação de Langevin na forma

$$
\frac{\partial}{\partial t} \delta \boldsymbol{\alpha}=-\mathbb{A} \delta \boldsymbol{\alpha}+\mathbb{D}^{1 / 2} \boldsymbol{\eta}(t),
$$

com

$$
\mathbb{A}=\left[\begin{array}{cccccc}
-\gamma_{1}\left(1-i \Delta_{1}\right) & -2 \epsilon^{*} \bar{\alpha}_{3} \bar{\alpha}_{4} & -2 \epsilon^{*} \bar{\alpha}_{1}^{*} \bar{\alpha}_{4} & 0 & -2 \epsilon^{*} \bar{\alpha}_{1}^{*} \bar{\alpha}_{3} & 0 \\
-2 \epsilon \bar{\alpha}_{3}^{*} \bar{\alpha}_{4}^{*} & -\gamma_{1}\left(1+i \Delta_{1}\right) & 0 & -2 \epsilon \bar{\alpha}_{1} \bar{\alpha}_{4}^{*} & 0 & -2 \epsilon \bar{\alpha}_{1} \bar{\alpha}_{3}^{*} \\
2 \epsilon \bar{\alpha}_{1} \bar{\alpha}_{4}^{*} & 0 & -\gamma(1-i \Delta) & 0 & 0 & \epsilon \bar{\alpha}_{1}^{2} \\
0 & 2 \epsilon^{*} \bar{\alpha}_{1}^{*} \bar{\alpha}_{4} & 0 & -\gamma(1+i \Delta) & \epsilon^{*} \bar{\alpha}_{1}^{* 2} & 0 \\
2 \epsilon \bar{\alpha}_{1} \bar{\alpha}_{3}^{*} & 0 & 0 & \epsilon \bar{\alpha}_{1}^{2} & -\gamma(1-i \Delta) & 0 \\
0 & 2 \epsilon^{*} \bar{\alpha}_{1}^{*} \bar{\alpha}_{3} & \epsilon^{*} \bar{\alpha}_{1}^{* 2} & 0 & 0 & -\gamma(1+i \Delta)
\end{array}\right]
$$

e

$$
\mathbb{D}=\left[\begin{array}{cccccc}
0 & \gamma_{1} & 0 & 0 & 0 & 0 \\
\gamma_{1} & 0 & 0 & 0 & 0 & 0 \\
0 & 0 & 0 & \gamma & 0 & 0 \\
0 & 0 & \gamma & 0 & 0 & 0 \\
0 & 0 & 0 & 0 & 0 & \gamma \\
0 & 0 & 0 & 0 & \gamma & 0
\end{array}\right]
$$

onde desta vez, fatoramos as flutuações $\delta \boldsymbol{\alpha}$, da matriz de arrasto $\mathbb{A}$. Notamos que, apesar de considerar a cavidade insensível aos campos gerados, informação contida na dessintonia e na constante $\gamma$, ainda fazemos distinção de seus modos. De fato, a dinâmica das intensidades dos modos são semelhantes, e portanto seus valores podem ser estudados sem levar em consideração informações dos modos individuais. No entanto, o mesmo não acontece para as flutuações, e devemos estudá-las separadamente.

Como vimos anteriormente, a descrição do campo eletromagnético é convenientemente feita através de suas variáveis canônicas: as quadraturas de amplitude e de fase. Portanto é razoável expressar as equações de dinâmica em termos destas variáveis. Podemos fazer essa passagem definindo os objetos que definem os modos como

$$
\alpha_{\imath} \equiv p_{\imath} \exp i \phi_{\imath},
$$

onde $p_{\imath}$ simboliza a amplitude, e $\phi_{\imath}$ a fase do modo $\imath$. Para os campos sinal e complementar temos $p_{3}=p_{4}=p$. 
Diferenciando a expressão (4.5.5) obtemos

$$
\delta \alpha_{\imath}=\exp \left(i \phi_{\imath}\right)\left(\delta p_{\imath}+i \delta q_{\imath}\right)
$$

onde $\delta p_{\imath}$ representa a flutuação na quadratura de amplitude e $\delta q_{\imath}$ a flutuação na quadratura de fase. Definimos também as flutuações estocásticas como

$$
\eta_{k}(t)=\eta_{\imath}(t)+i \eta_{\jmath}(t), \quad \operatorname{com} \imath \neq \jmath,
$$

que obedecem à correlação temporal (4.3.4). A decomposição das flutuações estocásticas em termos de uma parte real e uma parte imaginária, sugere a atuações destas forças estocásticas sobre as flutuações nas quadraturas de amplitude e fase.

Substituindo os modos dos campos, na forma descrita pela relação (4.5.5), na equação de Langevin (4.5.2) com as matrizes de arrasto e difusão dadas por $\mathbb{A}$ e $\mathbb{D}$ respectivamente, e separando a parte real, que corresponde à flutuação na quadratura de amplitude, da parte imaginária, correspondente à flutuação na quadratura de fase, obtemos um conjunto de seis equações diferenciais estocásticas acopladas para as flutuações nas quadraturas

$$
\begin{array}{r}
\dot{\delta} p_{1}=-\gamma_{1}\left(\delta p_{1}+\Delta_{1} \delta q_{1}\right)-2 \gamma \beta\left(\delta p_{3}+\delta p_{4}-\Delta \delta q_{3}-\Delta \delta q_{4}\right)-2 \gamma \beta^{2}\left(\delta p_{1}+\Delta \delta q_{1}\right) \\
+\sqrt{2 \gamma_{1}} \eta_{1}(t) \\
\dot{\delta} q_{1}=-\gamma_{1}\left(\delta q_{1}-\Delta_{1} \delta p_{1}\right)-2 \gamma \beta\left(\delta q_{3}+\delta q_{4}+\Delta \delta p_{3}+\Delta \delta p_{4}\right)-2 \gamma \beta^{2}\left(-\delta q_{1}+\Delta \delta p_{1}\right) \\
+\sqrt{2 \gamma_{1}} \eta_{2}(t) \\
\dot{\delta} p_{3}=-\gamma\left(\delta p_{3}+\Delta \delta q_{3}\right)+\gamma\left(\delta p_{4}-\Delta \delta q_{4}\right)+2 \gamma \beta\left(\delta p_{1}+\Delta \delta q_{1}\right)+\sqrt{2 \gamma} \eta_{3}(t) \\
\dot{\delta} q_{3}=-\gamma\left(\delta q_{3}-\Delta \delta p_{3}\right)-\gamma\left(\delta q_{4}+\Delta \delta p_{4}\right)+2 \gamma \beta\left(\delta q_{1}-\Delta \delta p_{1}\right)+\sqrt{2 \gamma} \eta_{4}(t) \\
\dot{\delta} p_{4}=-\gamma\left(\delta p_{4}+\Delta \delta q_{4}\right)+\gamma\left(\delta p_{3}-\Delta \delta q_{3}\right)+2 \gamma \beta\left(\delta p_{1}+\Delta \delta q_{1}\right)+\sqrt{2 \gamma} \eta_{5}(t) \\
\dot{\delta} q_{4}=-\gamma\left(\delta q_{4}-\Delta \delta p_{4}\right)-\gamma\left(\delta q_{3}+\Delta \delta p_{3}\right)+2 \gamma \beta\left(\delta q_{1}-\Delta \delta p_{1}\right)+\sqrt{2 \gamma} \eta_{6}(t)
\end{array}
$$

onde $\beta=p / p_{1}$ é a amplitude do campo gerado em unidades da amplitude do campo de bombeio. $\sqrt{2 \gamma_{1}}$ e $\sqrt{2 \gamma}$, representam os termos de acoplamento entre os modos do campo de bombeio, e dos campos gerados, respectivamente, com o reservatório. As equações dos campos gerados (4.5.10 - 4.5.13), são facilmente desacopladas definindo um novo par de variáveis

$$
\begin{aligned}
& \delta p_{ \pm}=\delta p_{3} \pm \delta p_{4}, \\
& \delta q_{ \pm}=\delta q_{3} \pm \delta q_{4} .
\end{aligned}
$$


É importante aqui, fazer distinção do acoplamento feito pelo próprio acoplador em questão, e as perdas espúrias modeladas pelo operador de aniquilação do banho. Para isso, escrevemos $\sqrt{2 \gamma}\left(\eta_{\jmath}(t)-\eta_{k}(t)\right) \equiv \sqrt{2 \gamma^{\prime}} \eta_{\jmath}(t)+\sqrt{2 \mu^{\prime}} \eta_{k}(t)$, com $\jmath \neq k$. A atuação do divisor de feixes de fibra sobre os modos dos campos é dada por $\sqrt{2 \gamma^{\prime}}$, e a ação das perdas gerais, estão separadas no fator $\sqrt{2 \mu^{\prime}}$. Com estas considerações obtemos, no espaço de soma e subtração, quatro equações diferenciais estocásticas desacopladas

$$
\begin{gathered}
\dot{\delta} p_{-}=-2 \gamma \delta p_{-}+\sqrt{2 \gamma^{\prime}} \eta_{1}(t)+\sqrt{2 \mu^{\prime}} \eta_{2}(t), \\
\dot{\delta} q_{-}=2 \gamma \Delta \delta p_{-}+\sqrt{2 \gamma^{\prime}} \eta_{3}(t)+\sqrt{2 \mu^{\prime}} \eta_{4}(t), \\
\dot{\delta} p_{+}=-2 \gamma \Delta \delta q_{+}+4 \gamma \beta\left(\delta p_{1}+\Delta \delta q_{1}\right)+\sqrt{2 \gamma^{\prime}} \eta_{5}(t)+\sqrt{2 \mu^{\prime}} \eta_{6}(t), \\
\dot{\delta} q_{+}=-2 \gamma \delta q_{+}+4 \gamma \beta\left(\delta q_{1}-\Delta \delta p_{1}\right)+\sqrt{2 \gamma^{\prime}} \eta_{7}(t)+\sqrt{2 \mu^{\prime}} \eta_{8}(t) .
\end{gathered}
$$

A descrição das equações de dinâmica no domínio temporal contém informações dispersas no espaço de frequências. Como vimos, o analisador de espectro realiza medidas no espaço de frequências, em um intervalo delimitado pelo seu filtro eletrônico. Portanto, é interessante explorar o comportamento das flutuações dos campos em um intervalo estreito de frequências, e relacionar as grandezas que são passíveis de serem medidas com as informações físicas provenientes da teoria.

\subsection{Espectro de flutuações}

Um sinal medido no domínio temporal, a menos que este seja gerado por um equipamento com a capacidade de gerar sinais com frequências únicas, como um gerador de sinal por exemplo, será composto por frequências principais, ou modos principais de oscilação, responsáveis pelo valor médio da amplitude, mais flutuações de amplitude e fase em torno do valor médio. Estas flutuações podem ser consideradas como sendo geradas por outros modos de oscilações da fonte principal, ou por fontes secundárias acopladas com a fonte principal, existindo neste caso, correlações entre os diversos modos. As flutuações também podem ser causadas por oscilações independentes da origem do sinal principal, e neste caso não haverá correlações.

Dessa forma, a melhor coisa a se fazer é decompor no espaço de Fourier as diversas componentes, que representam os diversos modos de oscilação. Isso nos traz a ideia de investigar o espectro de Fourier das flutuações, ou mais realisticamente, os valores médios das flutuações no espectro de frequências após muitos ciclos de medidas. A média tomada sobre o espectro de Fourier das flutuações está diretamente relacionada com as correlações espectrais dos modos de oscilação. A decomposição no espaço de Fourier de um sinal temporal proveniente de um processo físico, 
definido de $t=0$ até $t=\tau$, pode ser escrita como

$$
\tilde{X}(\omega)=\frac{1}{\sqrt{\tau}} \int_{0}^{\tau} d t \exp (i \omega t) X(t)
$$

onde o termo que acompanha a integral é responsável pela normalização. Sendo a função $X(t)$ real, temos $\tilde{X}^{*}(\omega)=\tilde{X}(-\omega)$. Um exemplo típico de um sinal temporal $X(t)$, e do módulo ao quadrado da quantidade $\tilde{X}(\omega)$, pode ser visto na figura 4.3. Um sinal temporal composto por uma oscilação principal na frequência $\Omega$, e flutuações de menor escala em frequências em torno deste valor, quando decompostos no espaço de Fourier, apresentam um perfil de distribuição simétrico com relação a zero, e centrado em $\Omega$.
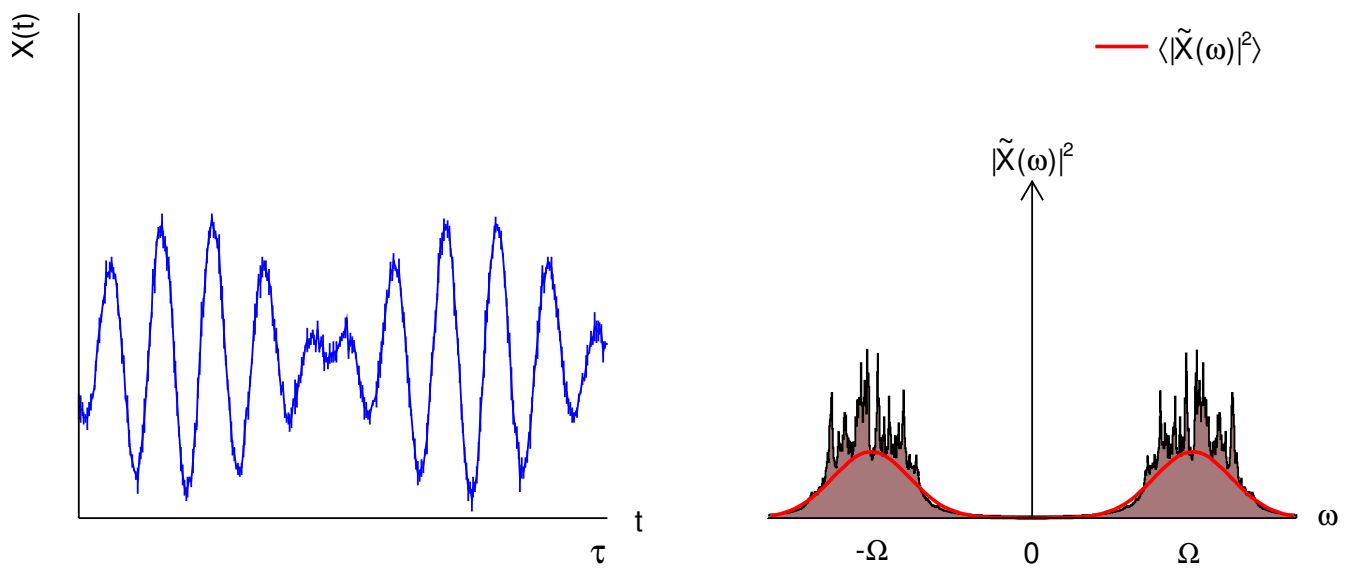

Figura 4.3: A figura da esquerda representa um sinal observado no tempo, originado de um processo físico com ruído. A figura da direita corresponde à decomposição do sinal temporal no espaço de Fourier. A curva em vermelho mostra a suavização do espectro através da média sobre muitos ciclos de medidas.

Se efetuarmos não uma, mas muitas medidas do sinal temporal, poderemos calcular uma média sobre várias medidas. Por não ser uma grandeza real, o objeto $\tilde{X}(\omega)$ não pode ser diretamente medido, portanto, é conveniente tomar a média sobre o módulo ao quadrado desta função,

$$
\left\langle|\tilde{X}(\omega)|^{2}\right\rangle=\frac{1}{\tau} \int_{0}^{\tau} d t_{1} d t_{2} \exp \left[i \omega\left(t_{2}-t_{1}\right)\right]\left\langle X\left(t_{1}\right) X\left(t_{2}\right)\right\rangle
$$

Na situação estacionária, os campos perderam qualquer referência da origem dos tempos, e não há nada de especial que tenha ocorrido neste instante. A consequência disto é que não há um ponto especial no tempo, e as medidas de correlações dependem apenas da diferença temporal $t_{2}-t_{1}$. É conveniente então, definir duas novas variáveis, de soma e subtração dos 
tempos. Como o argumento da exponencial depende apenas da diferença dos tempos, apenas a integração na variável de subtração nos interessa.

É necessário neste ponto, fazer alguns comentários a respeito da função de correlação temporal $\left\langle X\left(t_{1}\right) X\left(t_{2}\right)\right\rangle$. Tal função informa a existência de correlações temporais entre os modos dos campos em dois instantes de tempo diferentes $t_{1}$ e $t_{2}$. Obviamente a função de correlação deve ser limitada no espaço de subtração, uma vez que não deve haver correlações entre as flutuações de amplitude e fase de dois fotóns gerados em um intervalo de tempo muito distante. Em particular, quando analisamos correlações entre fótons gerados em um meio não linear dentro de uma cavidade, como a discutida na seção 3.3, o intervalo da janela temporal na qual é possível medir correlações, é fixado pela largura de linha da cavidade.

Portanto, a integral na variável de subtração pode ser estendida a valores tão grandes quanto se queira, uma vez que, para valores de tempo maiores do que o permitido pelo sistema, a função de correlação é nula

$$
\left\langle|\tilde{X}(\omega)|^{2}\right\rangle=\lim _{\tau \rightarrow \infty} \int_{-\tau}^{\tau} d t \exp (i \omega t)\langle X(t) X(0)\rangle,
$$

e o cálculo da integral na variável de soma resulta em $\tau$. A equação (4.6.3) nada mais é do que o teorema de Wiener Khinchin, já empregado na seção 2.2. O teorema diz que: a média do módulo ao quadrado da transformada de Fourier de um sinal medido resultante de um processo envolvendo ruído tende à transformada de Fourier da função de correlação no limite em que $\tau$ vai ao infinito. Essa relação é muito importante, porque associa a grandeza medida no experimento; o lado esquerdo da expressão, com a quantidade importantes na teoria, a função de correlação. Integrando em $\omega$ o lado direito da equação (4.6.3), e dividindo por $2 \pi$ obtemos

$$
\int_{-\infty}^{\infty} \frac{d \omega}{2 \pi}\langle\tilde{X}(-\omega) \tilde{X}(\omega)\rangle=\left\langle X^{2}\right\rangle
$$

Assim, sob condições estacionárias, a menos de um fator de $2 \pi$, a integral realizada sobre a média do espectro de flutuação corresponde ao valor esperado do quadrado da quantidade $X$.

\subsection{Espectro de compressão}

Com as ideias acima discutidas, é conveniente definirmos novamente a densidade de ruído espectral $S_{X}\left(\omega, \omega^{\prime}\right)$ como

$$
\left\langle X(\omega) X\left(-\omega^{\prime}\right)\right\rangle=2 \pi \delta\left(\omega-\omega^{\prime}\right) S_{X}\left(\omega, \omega^{\prime}\right)
$$


que define as correlações entre modos distintos no espaço de frequência.

Com essa quantidade em mãos, devemos calculá-la para as flutuações de amplitude e fase, no espaço de soma e subtração, dos campos gerados (4.5.15 - 4.5.18), para duas condições distintas. A primeira, quando estamos abaixo do limiar oscilação. Neste caso, não há campos gerados e obtemos informações sobre as correlações entre os modos de vácuo. Na segunda condição, acima do limiar de oscilação, a existência de novos campos distanciados de um FSR, nos permite analisar as correlação entre estes modos de frequências específicas, agora com um número significativo de fótons.

\subsubsection{Acima do limiar de oscilação}

Acima do limiar, há geração dos campos sinal e complementar, com um número significativo de fótons. Devido a este grande número de fótons gerados, um fluxo contínuo de fótons atinge o fotodiodo, estabelecendo uma corrente estacionária na eletrônica de amplificação que chega ao analisador de espectro. Dessa forma, é satisfeita a condição imposta sobre situações estacionárias de medida, discutida na seção anterior. Sendo assim, podemos usar diretamente a equação (4.6.4) na forma da (4.7.1). Definimos a subtração das flutuações de amplitude e fase, decompostas no espaço de Fourier, como

$$
\delta p_{-}(t)=\int_{-\infty}^{\infty} \frac{d \omega}{2 \pi} \exp (i \omega t) \delta p_{-}(\omega),
$$

e

$$
\delta q_{-}(t)=\int_{-\infty}^{\infty} \frac{d \omega}{2 \pi} \exp (i \omega t) \delta q_{-}(\omega) .
$$

As equações de dinâmica para estas variáveis no domínio temporal são equações diferenciais temporais estocásticas, (4.5.15) e (4.5.16). No espaço de Fourier, estas equações diferenciais estocásticas se transformam em equações algébricas estocásticas

$$
\begin{aligned}
& i \omega \delta p_{-}(\omega)=-2 \gamma \delta p_{-}(\omega)+\sqrt{2 \gamma^{\prime}} \eta_{1}(\omega)+\sqrt{2 \mu^{\prime}} \eta_{2}(\omega) \\
& i \omega \delta q_{-}(\omega)=2 \gamma \Delta \delta p_{-}(\omega)+\sqrt{2 \gamma^{\prime}} \eta_{3}(\omega)+\sqrt{2 \mu^{\prime}} \eta_{4}(\omega)
\end{aligned}
$$

onde definimos também as forças estocásticas no espaço de Fourier, sendo agora, $\delta$ correlacionada em frequência $\left\langle\eta_{\imath}^{*}\left(\omega^{\prime}\right) \eta_{\jmath}(\omega)\right\rangle=\delta_{\imath \jmath} \delta\left(\omega^{\prime}+\omega\right)$, com $\imath \neq \jmath$. O formalismo de entrada e saída da cavidade é dado por [55]

$$
\begin{aligned}
& \delta p_{-}^{\text {out }}=-\eta_{1}(\omega)+\sqrt{2 \gamma^{\prime}} \delta p_{-}^{\text {in }}(\omega), \\
& \delta q_{-}^{\text {out }}=-\eta_{3}(\omega)+\sqrt{2 \gamma^{\prime}} \delta q_{-}^{\text {in }}(\omega),
\end{aligned}
$$


onde $\delta p_{-}^{i n}(\omega)$ e $\delta q_{-}^{i n}(\omega)$ são os modos intracavidade, dados por (4.7.4) e (4.7.5).

Para uma cavidade ideal, sem perdas incontroláveis $(\mu=0)$, podemos encontrar o espectro de compressão para as flutuações de saída, inserindo (4.7.6) e (4.7.7) em (4.7.1),

$$
S p_{-}(\Omega)=1-\frac{4 \gamma^{\prime} / \gamma^{2}}{4+\Omega^{2}}\left(2 \gamma-\gamma^{\prime}\right)
$$

$\mathrm{e}$

$$
S q_{-}(\Omega)=1+\frac{4 \gamma^{\prime 2} / \gamma^{2}}{\Omega^{2}}\left(1+\frac{4 \Delta^{2}}{4+\Omega^{2}}\right)
$$

onde $\gamma$ é a largura de linha da cavidade, $\gamma^{\prime}$ é o acoplamento de entrada e saída dos modos através do acoplador de fibras, e $\Omega$ é a frequência de análise em unidades de largura de linha da cavidade. A figura 4.4 revela no espaço de subtração uma redução no ruído de amplitude

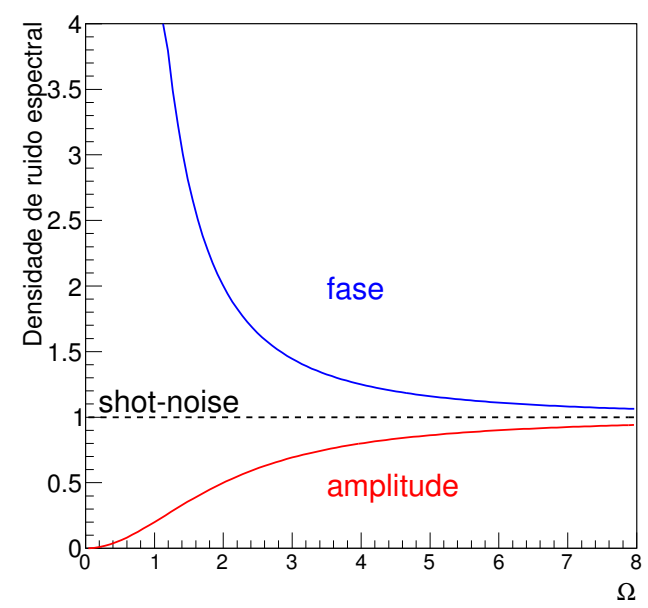

Figura 4.4: A figura representa graficamente, no espaço de subtração, o cálculo feito para densidade de ruído espectral das flutuações de amplitude e fase dos campos gerados. Consideramos a situação ideal, sem perdas incontroláveis $(\mu=0)$, com dessintonia nula e $\gamma=\gamma^{\prime}$.

dos campos gerados abaixo do shot-noise. Na condição ideal, isto é, sem perdas espúrias e a cavidade criticamente acoplada, é possível observar compressão nesta quantidade em até seis larguras de linha da cavidade, valor necessário para a observação de até $10 \%$ de compressão.

\subsubsection{Abaixo do limiar de oscilação}

Nesta situação, não há geração dos campos sinal e complementar, logo estes modos são caracterizados como modos de vácuo. Modos de vácuo, nada mais são do que modos ocupados por 
uma quantidade pequena de fótons, tendo um valor médio de campo nulo. Apesar de não ser possível realizar qualquer medição sobre seus valores médios, com a deteç̧ão em cavidade, ainda é possível obter informações sobre suas flutuações, devido ao batimento entres estes modos e a portadora.

Matematicamente, informamos a ausência dos campos gerados nas equações de dinâmica fazendo $\bar{\alpha}_{3}$ e $\bar{\alpha}_{4}$ nulos. Note que esta condição não é equivalente a se fazer $\beta=0$ nas equações (4.5.9 - 4.5.13), uma vez que assumimos a existência dos modos $\alpha_{3}$ e $\alpha_{4}$ para chegar naquelas equações. De fato, devemos retornar à equação de Langevin e rescrever a matriz de arrasto fazendo os valores esperados dos modos gerados iguais a zero. Repetindo as etapas que nos levaram às equações de dinâmica linearizadas no espaço de soma e subtração, temos

$$
\begin{gathered}
\dot{\delta} X_{-}=-\gamma\left(1+\sigma^{4}\right) \delta X_{-}-\gamma \Delta\left(1-\sigma^{4}\right) \delta Y_{-}+\sqrt{2 \gamma^{\prime}} \eta_{1}(t)+\sqrt{2 \mu^{\prime}} \eta_{2}(t), \\
\dot{\delta} Y_{-}=-\gamma\left(1-\sigma^{4}\right) \delta Y_{-}+\gamma \Delta\left(1+\sigma^{4}\right) \delta X_{-}+\sqrt{2 \gamma^{\prime}} \eta_{3}(t)+\sqrt{2 \mu^{\prime}} \eta_{4}(t), \\
\dot{\delta} X_{+}=-\gamma\left(1-\sigma^{4}\right) \delta X_{+}-\gamma \Delta\left(1+\sigma^{4}\right) \delta Y_{+}+\sqrt{2 \gamma^{\prime}} \eta_{5}(t)+\sqrt{2 \mu^{\prime}} \eta_{6}(t), \\
\dot{\delta} Y_{+}=-\gamma\left(1+\sigma^{4}\right) \delta Y_{+}+\gamma \Delta\left(1-\sigma^{4}\right) \delta X_{+}+\sqrt{2 \gamma^{\prime}} \eta_{7}(t)+\sqrt{2 \mu^{\prime}} \eta_{8}(t),
\end{gathered}
$$

onde $X_{ \pm}$e $Y_{ \pm}$são variáveis canonicamente conjugadas que descrevem os estados de vácuo nos espaços de soma e subtração, e $\sigma \equiv p_{1} / p_{1, \text { limiar }}$ é definido com a fração da intensidade do campo de bombeio intracavidade com relação à intensidade do limiar de oscilação (4.4.11).

Diferentemente do que ocorre com as equações de dinâmica quando há campos gerados, abaixo do limiar de oscilação as equações para os modos de soma e subtração continuam acopladas. A maneira mais simples de desacoplá-las é considerar a situação de dessintonia nula, fazendo $\Delta=0$. Nesta condição, decompomos novamente as flutuações temporais no espaço de Fourier, semelhante às equações (4.7.2) e (4.7.3), e obtemos a descrição algébrica destas quantidades

$$
\begin{aligned}
i \omega \delta X_{-}(\omega) & =-\gamma\left(1+\sigma^{4}\right) \delta X_{-}(\omega)+\sqrt{2 \gamma^{\prime}} \eta_{1}(\omega)+\sqrt{2 \mu^{\prime}} \eta_{2}(\omega), \\
i \omega \delta Y_{-}(\omega) & =-\gamma\left(1-\sigma^{4}\right) \delta Y_{-}(\omega)+\sqrt{2 \gamma^{\prime}} \eta_{3}(\omega)+\sqrt{2 \mu^{\prime}} \eta_{4}(\omega), \\
i \omega \delta X_{+}(\omega) & =-\gamma\left(1-\sigma^{4}\right) \delta X_{+}(\omega)+\sqrt{2 \gamma^{\prime}} \eta_{5}(\omega)+\sqrt{2 \mu^{\prime}} \eta_{6}(\omega), \\
i \omega \delta Y_{+}(\omega) & =-\gamma\left(1+\sigma^{4}\right) \delta Y_{+}(\omega)+\sqrt{2 \gamma^{\prime}} \eta_{7}(\omega)+\sqrt{2 \mu^{\prime}} \eta_{8}(\omega) .
\end{aligned}
$$

As expressões abaixo se referem às densidades de ruído espectrais para as flutuações das quadraturas conjugadas em uma cavidade ideal, sem perdas, com dessintonia nula e abaixo do limiar 
de oscilação

$$
\begin{gathered}
S X_{-}(\Omega)=1-\frac{4 \gamma^{\prime} / \gamma\left[\left(1+\sigma^{4}\right)-\gamma^{\prime} / \gamma\right]}{\left(1+\sigma^{4}\right)^{2}+\Omega^{2}}, \\
S Y_{-}(\Omega)=1-\frac{4 \gamma^{\prime} / \gamma\left[\left(1-\sigma^{4}\right)-\gamma^{\prime} / \gamma\right]}{\left(1-\sigma^{4}\right)^{2}+\Omega^{2}}, \\
S X_{+}(\Omega)=1-\frac{4 \gamma^{\prime} / \gamma\left[\left(1-\sigma^{4}\right)-\gamma^{\prime} / \gamma\right]}{\left(1-\sigma^{4}\right)^{2}+\Omega^{2}}, \\
S Y_{+}(\Omega)=1-\frac{4 \gamma^{\prime} / \gamma\left[\left(1+\sigma^{4}\right)-\gamma^{\prime} / \gamma\right]}{\left(1+\sigma^{4}\right)^{2}+\Omega^{2}} .
\end{gathered}
$$

A representação gráfica destas expressões pode ser vista na figura 4.5. Abaixo do limiar de
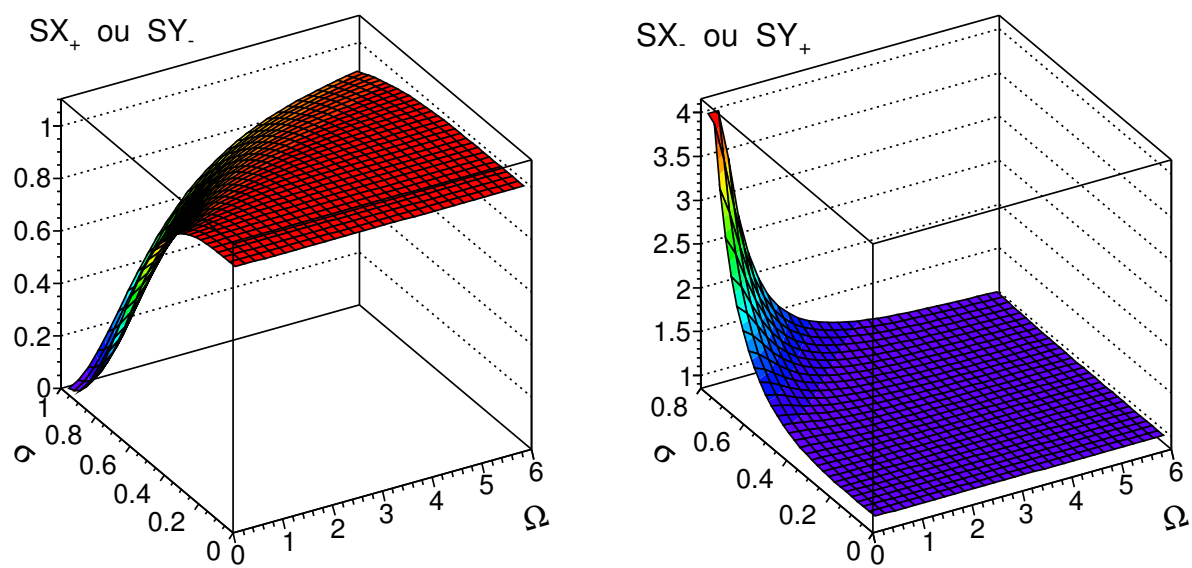

Figura 4.5: Redução do ruído de vácuo em uma quadratura, nos espaços de soma e subtração, abaixo do limite quântico padrão, com respectivo excesso de ruído na quadratura conjugada.

oscilação, obtemos quatro equações diferenciais estocásticas acopladas no espaço de soma e subtração dos campos sinal e complementar. Apesar da ausência de fótons em quantidade suficiente que caracterize a existência de novos campos gerados, com valores médios mensuráveis, ainda podemos estudar as flutuações destes modos a partir do processo de linearização dos campos. Fisicamente, estas quantidades representam o comportamento das flutuações dos estados de vácuo dos modos acoplados com a cavidade, que também podem sofrer alterações quando na presença de um meio não linear.

O caso mais simples de analisar o processo é na situação de dessintonia nula, isto é, os modos estão em ressonância com a cavidade. Nesta condição, as equações de dinâmica se desacoplam 
e podemos tratá-las facilmente no espaço de frequências.

Do tratamento estocástico desenvolvido neste capítulo, somos levados a procurar compressão de ruído, no regime de oscilação, na diferença entre as quadraturas de amplitude dos campos gerados. Consequentemente, aguardamos um excesso de ruído na quadratura conjugada, fase. Uma situação parecida com esta ocorre quando a intensidade do campo de bombeio é pequena, isto é, insuficiente para iniciar a geração dos campos. Neste caso, aguardamos também uma compressão no ruído de amplitude quando analisamos a diferença dos modos de vácuo, $\alpha_{3}$ e $\alpha_{4}$, acoplados com a cavidade.

Ainda abaixo do limiar, uma alternativa é olhar para a soma dos ruídos dos modos acoplados. Desta vez aguardamos uma medida com excesso de ruído em uma quadratura, e uma redução no ruído da quadratura conjugada, abaixo do limite quântico padrão.

A existência de fótons nos modos de vácuo depende da taxa de conversão dos fótons do bombeio para estes modos. Então, obviamente, o número de fótons presentes nestes modos dependem da intensidade do campo de bombeio. Esta ideia reflete na dependência das soluções com $\sigma$. É natural que, para uma intensidade de bombeio bem abaixo do limiar, esperamos que haja um menor efeito de compressão nas bandas laterais, já que a eficiência do processo para gerar as correlações entre os modos necessita da existência dos fótons do bombeio. Então, a máxima correlação possível de ser medida caminha no sentido de maximizar $\sigma$, isto é, atingir a unidade. Uma vez atingido este valor, o processo de geração dos campos se inicia, e um outro efeito interessante é observado, a biestabilidade.

Uma vez atingido o limiar de oscilação, a taxa de conversão de fótons através do processo de mistura de quatro ondas se estabiliza, e é desnecessário aumentar a intensidade do campo de bombeio. O limiar de oscilação é atingido quando o ganho, devido à conversão paramétrica, supera as perdas na cavidade em uma volta. Desta forma, para uma cavidade com perdas em demasia, o limiar de oscilação será maior e as correlações reduzidas. O motivo disso é que, com grandes perdas na cavidade, o ganho deverá ser maior para superá-las, e a dissipação de fótons para o reservatório reduz as correlações, simplesmente porque a probabilidade de que um dos fótons gêmeos seja absorvido pelo reservatório é maior, reduzindo a taxa de medição dos feixes gêmeos pelo detector. 



\section{Capítulo 5}

\section{Descrição Experimental}

Este capítulo trata das medidas realizadas ao longo do período de mestrado. Iniciamos com uma breve introdução teórica do espalhamento Brillouin estimulado, e também do espalhamento por ondas acústicas, fônons. Mostramos as primeiras medidas realizadas no sentido de determinar o limiar de oscilação Brillouin. Comentamos como este efeito parasita pode mascarar as medidas de ruído realizadas ao nível de shot-noise.

Seguindo, fazemos uma descrição pictórica e em palavras do aparato experimental proposto, e apresentamos as principais medidas de ruído.

\subsection{Espalhamento Brillouin}

Modos mecânicos de vibração do núcleo da fibra óptica são termicamente excitados em temperatura ambiente. Alguns destes modos podem modular o índice de refração da fibra, assim, modulando a fase do campo. Há também a excitação de fônons que se propagam transversalmente. Este efeito é conhecido como GAWBS, do inglês (Guided Acoustic Wave Brillouin Scattering).

\subsubsection{Espalhamento Brillouin estimulado}

De acordo com a teoria clássica, o espalhamento Brillouin estimulado (SBS) pode ser descrito como uma interação não linear entre a luz e as ondas acústicas. No modelo quântico, um fóton do bombeio é aniquilado para criar um fóton com energia menor, onda Stokes, e um fônon. Também é possível criar um fóton com energia maior do que o fóton de bombeio, denominada 
de onda Anti-Stokes, ver figura 5.1. Em ambos os casos, a energia e o momento são conservados [2].

$$
\begin{array}{ll}
\hbar \omega_{1}=\hbar \omega_{s c} \pm \hbar \omega_{b r} \\
\hbar \mathbf{k}_{1}=\hbar \mathbf{k}_{s c} \pm \hbar \mathbf{k}_{b r}
\end{array} \begin{cases}+ & \text { Stokes } \\
- & \text { Anti-Stokes }\end{cases}
$$

Na relação acima, $\omega_{1}$ é a frequência do campo de bombeio, $\omega_{s c}$ é a frequência do fóton originado do processo, e $\omega_{b r}$ é a frequência da onda acústica. Os vetores de onda destes campos são os vetores $\mathbf{k}_{1}, \mathbf{k}_{s c}$ e $\mathbf{k}_{b r}$, respectivamente. Obtemos pela conservação da energia e do momento

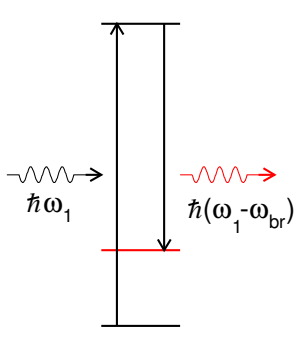

Espalhamento Stokes

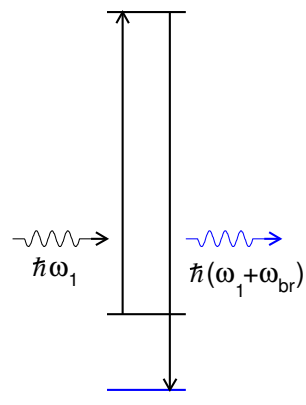

Espalhamento

Figura 5.1: Espalhamento Brillouin estimulado. No espalhamento Stokes, o campo de bombeio origina um campo com energia menor que se propaga no sentido contrário ao campo de bombeio, denominado de Stokes. No espalhamento do tipo Anti-Stokes, o campo criado é composto por fótons com energia maior que os do campo de bombeio.

$$
\omega_{b r} \approx 2 v\left|k_{1}\right| \sin (\theta / 2)
$$

onde $\theta$ é o ângulo entre o fóton do bombeio e o fóton Stokes, e $v$ é a velocidade do som na fibra. Como a fibra possui somente duas direções de propagação, para frente ou para trás, vemos que ocorre espalhamento Brillouin estimulado apenas para trás $(\theta=\pi)$.

Quando o limiar de oscilação do espalhamento Brillouin é alcançado, grande parte da energia do campo de bombeio será transferida para a onda Stokes, causando um efeito de saturação da energia do feixe de bombeio. O deslocamento Stokes, com relação ao campo de bombeio, é de aproximadamente $10 \mathrm{GHz}$, com uma largura de linha de 10MHz. O limiar de oscilação está 
associado principalmente com o campo propagante e com a estrutura da fibra óptica. Para um bombeio CW (Continuous Wave), o limiar pode ser tão baixo quanto $1 \mathrm{~mW}$. Em contraste, para feixes pulsados, com taxa de repetição inferior ao tempo de existência dos fônons, o que implica em uma largura espectral efetiva superior à largura espectral do SBS, o ganho do espalhamento Brillouin é reduzido. De fato, a largura da onda Stokes é caracterizada pelo ganho espectral do SBS, e está relacionada com o tempo de vida dos fônons na fibra, $\tau_{b r} \equiv \Gamma_{b r}^{-1} \approx 10 \mathrm{~ns}$. Como o espectro é relativamente estreito $(\sim 10 \mathrm{MHz})$, ele pode ser aproximado por um perfil Lorentziano, centrado na frequência de ressonância $\omega_{b r}$

$$
g_{b r}\left(\Delta_{b r}\right)=g_{p} \frac{\left(\Gamma_{b r} / 2\right)^{2}}{\Delta_{b r}^{2}+\left(\Gamma_{b r} / 2\right)^{2}},
$$

onde $\Delta_{b r}=\omega-\omega_{b r}$, é a distância em frequência ao pico Brillouin. A constante $g_{p}$ depende dos parâmetros de construção da fibra, como o material empregado, sua densidade, a elasticidade resultante, o índice de refração e a velocidade de propagação do som neste meio. Para fibras de sílica convencionais, o valor de $g_{p}$ é calculado em $5 \times 10^{-11} \mathrm{~m} / \mathrm{W}$.

Em um laser $\mathrm{CW}$, o ganho Brillouin é reduzido drasticamente quando a largura de linha do laser é superior à da onda Stokes. Isso acontece quando o laser de bombeio é multimodo, ou quando uma modulação de fase que varia rapidamente, em uma escala menor que o tempo de vida dos fônons, é aplicada a um laser monomodo. Sob este regime, mostra-se que, quando o comprimento de coerência do laser é maior que o comprimento de interação da onda Stokes, definido como a distância na qual a amplitude Stokes varia significativamente, a magnitude do SBS é reduzido por um fator $1+\delta_{b} / \delta_{b r}$, onde $\delta_{b}$ e $\delta_{s c}$ são as larguras de linha do campo de bombeio e da onda Stokes respectivamente [2].

O limiar de Brillouin também é alterado pelos parâmetros da fibra. Evidentemente, fibras maiores possuem um comprimento efetivo de interação da luz com a fibra maior, aumentando a probabilidade do fóton sofrer espalhamento, diminuindo o limiar. Algo semelhante ocorre com a área efetiva da fibra. Para fibras altamente não lineares, a região responsável pelo guiamento da luz é menor, deixando o diâmetro do feixe mais concentrado e aumentando a densidade óptica da luz. A consequência é uma densidade maior de fótons, aumentando também a probabilidade de que estes sofram espalhamento.

O limiar de oscilação pode ser estimado segundo a relação [48]

$$
\frac{g_{b r}(0) P_{b r}^{c r} L_{e f}}{A_{e f}} \approx 21,
$$

onde $P_{b r}^{c r}$ é a potência crítica para iniciar a geração de fótons Stokes. $L_{e f}$ e $A_{e f}$, são o comprimento e a área efetiva da fibra. Para fibras convencionais modernas, com poucas perdas, 


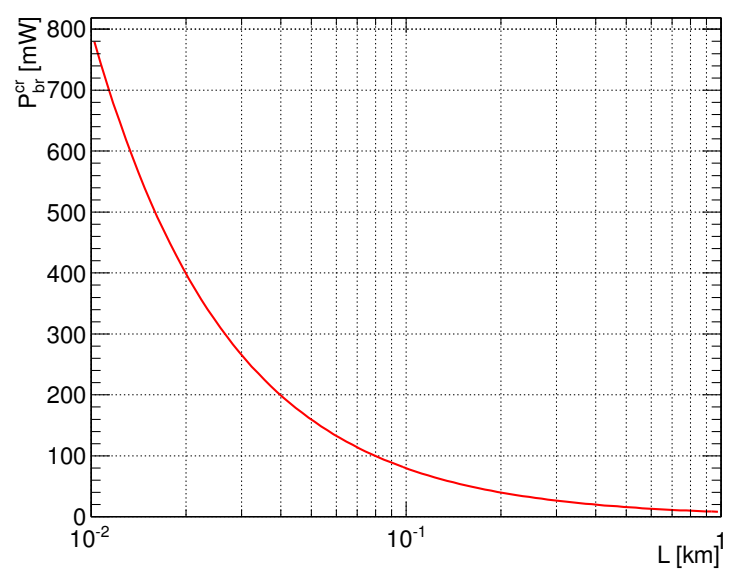

Figura 5.2: Este gráfico mostra a intensidade do limiar de oscilação do espalhamento Brillouin em função do comprimento $L$ da fibra. A área transversal foi medida em $A=1,9 \times 10^{-11} \mathrm{~m}^{2}$.

o comprimento efetivo pode ser aproximado pelo comprimento físico da fibra. Apenas para estimar o limiar, é comum considerar também a área efetiva como a área do núcleo. A figura 5.2 mostra o limiar do espalhamento Brillouin estimulado para uma fibra típica, altamente não linear, para diversos comprimentos. Enquanto que a figura 5.3, mostra um possível processo para a obtenção do limiar de oscilação Brillouin experimentalmente. O laser é acoplado com a fibra através de um circulador óptico. Observa-se a onda contra propagante Stokes por um analisador de espectro óptico, conectado diretamente ao circulador. A intensidade do laser é então aumentada gradualmente até o surgimento de um pequeno pico distanciando em aproximadamente $9,8 \mathrm{GHz}$, à direita do pico do bombeio em unidades de comprimento de onda. Uma vez iniciada a oscilação Brillouin, nota-se um efeito altamente não linear na amplitude. Para um pequeno aumento na intensidade do bombeio, o campo Stokes apresenta grandes flutuações na intensidade, aumentando exponencialmente em função da intensidade do bombeio.

Medimos o limiar de oscilação para uma fibra com 1,5km de comprimento, altamente não linear, com uma área transversal do núcleo de aproximadamente $1,9 \times 10^{-11} \mathrm{~m}^{2}$. O valor obtido foi de aproximadamente $7 \mathrm{dBm}$ na saída do laser. Em um ressoador, é de se esperar que este limiar seja menor, uma vez que as múltiplas reflexões sofridas pelo fóton aumentam a possibilidade deste sofrer espalhamento por fônon. 

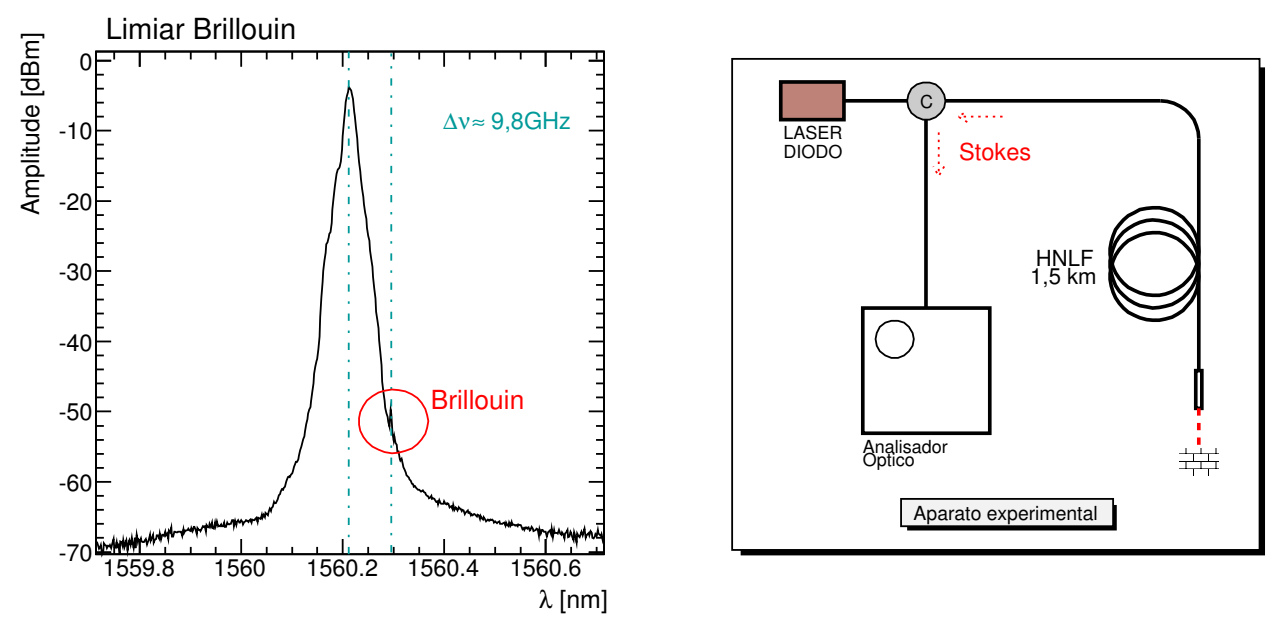

Figura 5.3: Obtenção experimental do limiar de oscilação do espalhamento Brillouin. Os dados obtidos no gráfico a esquerda se refere a uma medida realizada com o aparato experimental representado na figura da direita. A intensidade do laser de diodo é aumentada até o surgimento de um pequeno pico à direita do pico principal, representando o laser. O limiar obtido, para uma fibra HNLF de $1,5 \mathrm{~km}$, foi de $7 \mathrm{dBm}$. A distância entre os dois picos é de aproximadamente $0,08 \mathrm{~nm}$.

\subsubsection{GAWBS}

A equação (5.1.1) prediz corretamente a existência de fótons Stokes propagando no sentido contrário ao campo de bombeio - back scattering - devido ao espalhamento destes por fônons. No entanto, um modelo mais detalhado de vibrações termicamente induzidas no núcleo revelam um espalhamento espontâneo que pode ocorrer na mesma direção de propagação do campo de bombeio [45]. Isso se deve à natureza do guiamento das ondas acústicas na fibra, levando a um relaxamento nas regras de seleção dos vetores de onda. Este fenômeno é conhecido por GAWBS, do inglês (Guided Acoustic Wave Brillouin Scattering). O espectro de GAWBS pode ser estendido em múltiplas linhas, com largura de até $50 \mathrm{kHz}$, indo de $10 \mathrm{MHz}$ a $800 \mathrm{MHz}$. Resumidamente, estes espectros de linha correspondem à estrutura fina dos modos vibracionais, radiais e circunferenciais das ondas acústicas, que produzem constrição e dilatação do núcleo. O resultado desta oscilação do núcleo é causar modulações de fase e na polarização do campo propagante. A luz espalhada por este processo constitui uma fonte térmica de ruído em fibras ópticas, que apesar de sua menor magnitude com relação ao Brillouin estimulado, ainda pode acrescentar ruído significativo ao sistema.

Os modos vibracionais de um cilindro são caracterizados por perfis de deslocamentos transversos e um vetor de onda na direção paralela ao eixo [51]. Estes modos podem ser descritos de acordo 
com seu tipo de movimento, que podem ser radial, longitudinal, torsional, flexional, ou uma mistura deles [46]. Em frequências altas de modulação, o efeito do GAWBS é adicionar ruído nos modos das bandas laterais. GAWBS deve ser levado em conta em qualquer tipo de medida de ruído, principalmente naquelas relacionadas com as medidas de compressão nas quadraturas. Apesar de seu efeito ser quase insignificante com relação ao SBS, ele ainda pode mascarar medidas comparativas de ruído ao nível do limite quântico padrão.

A maneira de estudar a influência do GAWBS sobre o ruído do campo, guiado por uma fibra na configuração de cavidade, é descrever os modos mecânicos da fibra como um oscilador harmônico amortecido, com operadores de criação e aniquilação $\hat{b}_{G}^{\dagger}$ e $\hat{b}_{G}$ respectivamente, na frequência $\omega_{G}$. A atuação do processo de espalhamento sobre as equações de dinâmica, pode ser escrito na forma de uma Hamiltoniana de espalhamento do tipo

$$
\hat{H}_{G}=\hbar\left(\hat{a}_{A}^{\dagger} E+\hat{a}_{S} E^{*}\right) \hat{B}+h . c,
$$

onde

$$
\hat{B}=\tilde{R}^{*} \sum_{g} \hat{b}_{G}
$$

e $\tilde{R}$ é uma constante de acoplamento dimensional. Esta Hamiltoniana descreve o espalhamento dos fótons do bombeio em fótons Stokes e Anti-Sokes, representado pelos operadores $\hat{a}_{S}$ e $\hat{a}_{A}$ respectivamente. Acrescentada à equação mestra (4.1.6), juntamente com a Hamiltoniana que descreve o processo principal da mistura de quatro ondas em uma cavidade, podemos obter novamente as equações de dinâmica do sistema através do procedimento exposto no capítulo 4 . Na referência [43], mostra-se que o efeito do GAWBS sobre as variâncias dos modos das bandas laterais é acrescentar um termo de ruído

$$
\operatorname{var}_{\text {tot }}=\operatorname{var}_{\text {quantica }}+\frac{\beta(\Omega)}{\beta} \operatorname{var}_{G A W B S},
$$

onde o coeficiente $\beta(\Omega) / \beta$ reflete o perfil espectral do GAWBS na fibra, medido fora da cavidade. Em algumas fibras, a largura de linha dos picos espectrais pode ser bem estreita, existindo regiões entre dois picos em que a variância caia até o limite quântico. Estas regiões podem ser promissoras para a procura de compressão nas quadraturas.

\subsection{Caracterização instrumental}

Nesta seção apresentarei toda a instrumentação necessária para a realização prática da teoria desenvolvida no capítulo 4. 
Laser Durante o processo de construção e estabilização da cavidade testamos diversos lasers: Santec ECL 210, Agilent 81940A, Agilent 81600B e RIO (Orion Module). Os três primeiros, apesar de fornecerem uma boa potência de saída $(\sim 14,5 \mathrm{dBm})$, não tinham estabilidade em frequência nem largura de linha estreita o suficiente para manter uma cavidade estável, como pode ser visto na figura 5.4. Os modelos da Agilent, com largura de linha superior a $100 \mathrm{kHz}, \mathrm{e}$
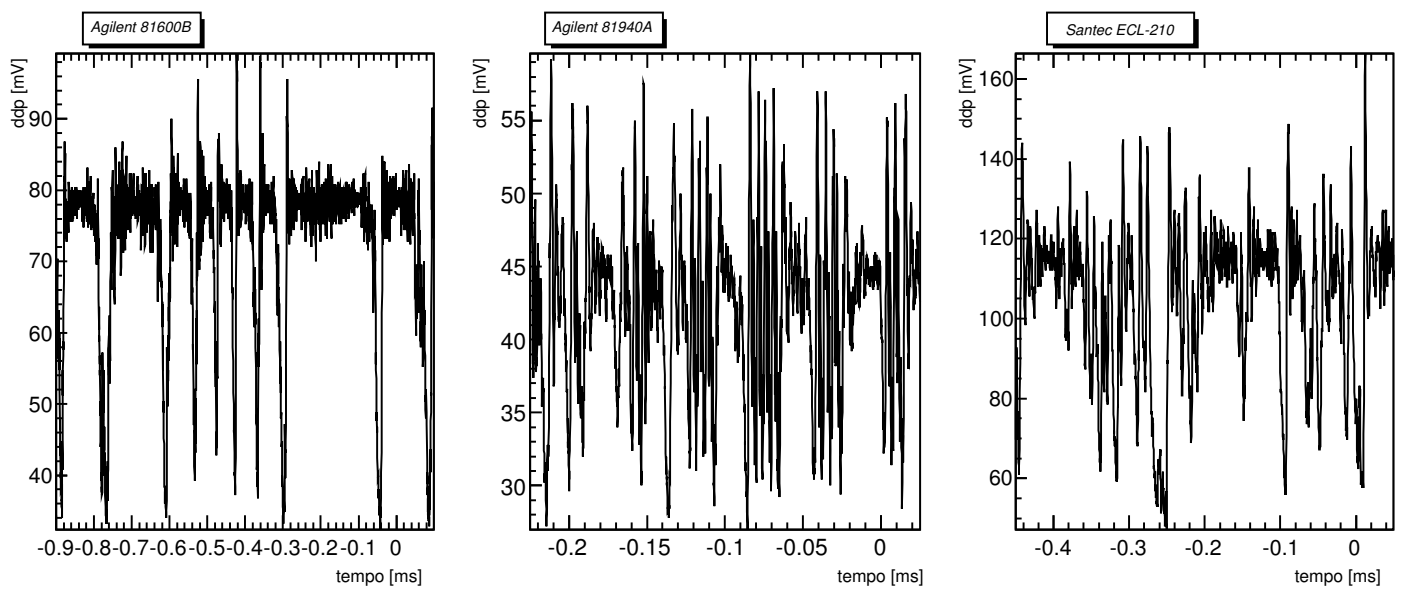

Figura 5.4: Comportamento da cavidade para diversos lasers. Os lasers da Santec e da Agilent não foram capazes de configurarem uma cavidade de centenas de metros estável.

estabilidade de $0,5 \mathrm{pm}$ por minuto, são capazes de varrer vários modos de uma cavidade de cem metros sem ao menos aplicar uma modulação sobre ela, cavidade parada. O laser da Santec apresentou o pior comportamento.

O que mais se adequou ao experimento proposto foi o laser da RIO, da REDFERN INTEGRATED OPTICS. O manual [1], informa que o laser fornece uma intensidade de até $20 \mathrm{~mW}$ na saída da fibra, e que possui ruídos de intensidade e fase muito baixos, largura de linha inferior a $3 \mathrm{kHz}$, excelente estabilidade em comprimento de onda e insensível a vibrações. A intensidade máxima medida na saída da fibra foi de $13 \mathrm{dBm}(\sim 20 \mathrm{~mW})$, com o pico em $1560 \mathrm{~nm}$. O comportamento de uma cavidade de $60 \mathrm{~m}$ bombeado por ele pode ser verificado na figura 3.5 .

O ruído de amplitude foi medido inicialmente através de uma medida de auto-homodinagem, descrita na seção 2.6, e mostramos realmente pouco ruído clássico nesta quadratura. No entanto, em uma medida do ruído de fase através da deteç̧ão com a cavidade de análise, o laser mostrou excesso de ruído nesta quadratura, como pode ser visto na figura $5.5^{1}$. O primeiro gráfico representa o sinal medido pelo analisador de espectro. Note que de $10 \mathrm{MHz}$ a $80 \mathrm{MHz}$, o shot-noise está a mais de 10dB acima do ruído eletrônico, região mais confiável do espectro.

\footnotetext{
${ }^{1}$ Os detalhes técnicos e procedimentos de medida serão descritos mais adiante.
} 

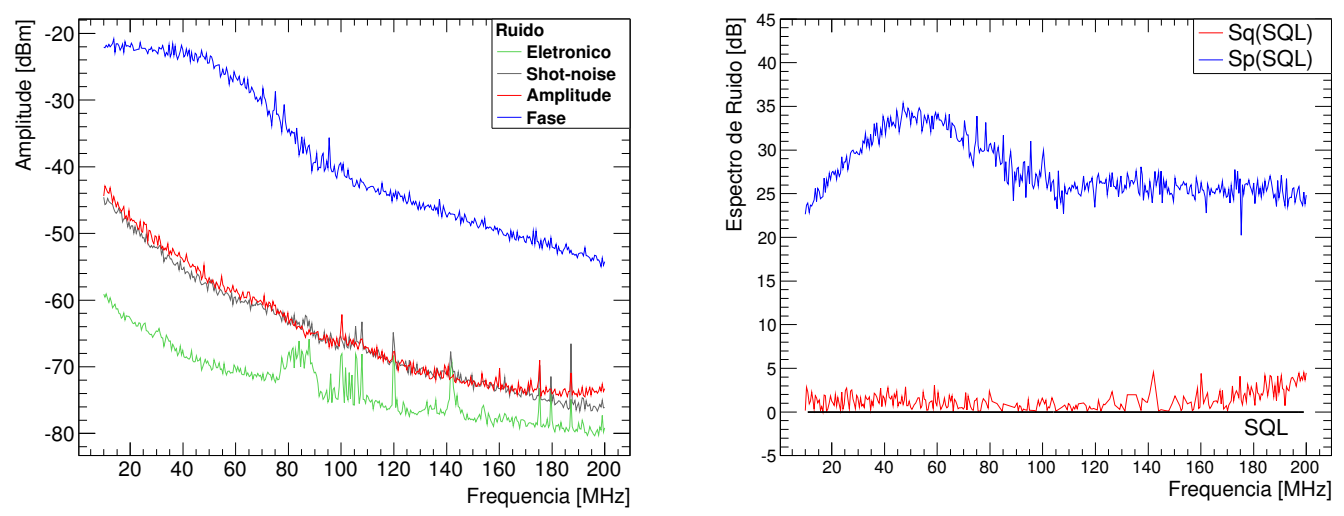

Figura 5.5: Medida de ruído do laser. As medidas foram feitas com a cavidade de análise. Apesar do ruído de intensidade ser próximo do shot-noise, a fase apresenta um excesso de ruído de mais de $20 \mathrm{~dB}$. A potência de luz iluminando cada fotodiodo era de aproximadamente $5 \mathrm{~mW}$.

Para frequências maiores, apesar de haver algumas regiões com grandes flutuações no ruído eletrônico, entre estas regiões conturbadas existem regiões confiáveis de medida de até $3 \mathrm{~dB}$ acima do eletrônico. Vale destacar que, nunca no grupo conseguimos medir espectros de ruído até 200MHz. O segundo gráfico mostra os níveis de ruído de amplitude e fase do laser com relação ao limite quântico padrão. Destaque para a quadratura de fase, com um excesso de ruído de 20dB acima do SQL, típico de um laser de diodo [59].

Durante todo o procedimento de medição, notamos um "drift" dos picos de reflexão da cavidade de fibra sempre para a mesma direção. Inicialmente pensamos que poderia ser a influência térmica da temperatura sobre a fibra, que poderia alterar seu comprimento, alterando o caminho óptico do campo guiado, e consequentemente, perturbando o FSR da cavidade. No entanto, em uma escala de tempo curta, da ordem de minutos, devido ao caráter aleatório das flutuações térmicas, isso causaria uma alteração também aleatória na distância entre dois picos de reflexão, aproximando-os ou afastando-os. Outra possibilidade seria a fibra sofrer com os valores médios da temperatura, que possui um comportamento contínuo em uma escala de horas. Mas neste caso, apesar de ser possível a observação do drift de apenas um pico de reflexão, em dois picos, observaríamos uma aproximação ou afastamento contínuo entre eles.

Contudo, o monitoramento de algumas horas dos parâmetros internos do laser, mostrou uma queda sistemática em sua temperatura, como pode ser visto na figura 5.6. Após uma hora e dez minutos de funcionamento o laser atinge uma certa estabilidade, mas seu valor médio apresenta uma ligeira queda com uma flutuação de aproximadamente 0,02\%. Esta queda constante na temperatura pode implicar uma mudança constante em uma direção na frequência do laser, causando o efeito observado na saída da cavidade. 


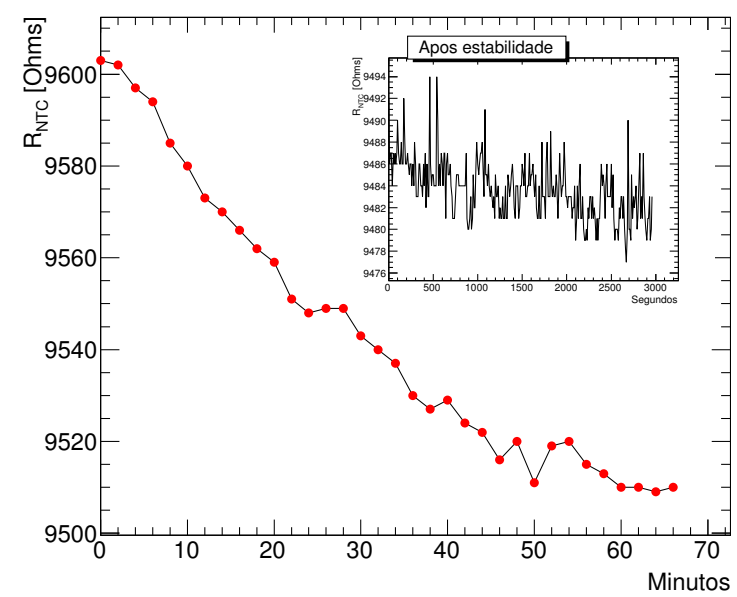

Figura 5.6: Monitoramento da flutuação térmica do laser. Este apresenta uma queda sistemática na temperatura interna. Mesmo após uma certa estabilidade, seu valor médio continua a cair lentamente.

Fibra óptica Dentre os vários tipos de fibras existentes atualmente, focamos nossas atenções nas fibras que possuem um alto fator de não linearidade e que apresentasse poucas perdas de propagação. O comprimento da fibra deve ser longo o suficiente para resultar em uma interação não linear significativa, mas não tão longa a ponto de atenuar muito o campo ao longo de sua propagação, de outra forma irá degradar a compressão. Procuramos fibras que apresentassem uma atenuação máxima da ordem de $0,5 \mathrm{~dB} / \mathrm{km}$. Os três principais parâmetros que devemos ter em mente: o fator de atenuação, a intensidade de limiar do espalhamento Brillouin, e o coeficiente de não linearidade. Observaremos compressão suficiente nas quadraturas, se a seguinte condição for alcançada

$$
\gamma I_{b} L_{e f} \geq 1
$$

para uma fibra com comprimento efetivo $L_{e f}$ sem estar configurada na forma de cavidade. A intensidade $I_{b}$ é a intensidade do campo de bombeio, e para uma maior eficiência no $4 \mathrm{WM}$, ela deve ser o mais próximo possível do limiar de oscilação do espalhamento Brillouin.

Como o nosso laser não tem uma intensidade muito grande, para satisfazer a condição (5.2.1) temos duas opções: aumentar o fator de não linearidade ou o comprimento da fibra. Atualmente, as fibras de cristais fotônico (microestruturadas) possuem um $\gamma$ de até $100 \mathrm{~W}^{-1} \mathrm{~km}^{-1}$. No entanto, estas fibras costumam ser sensíveis a deformações mecânicas e apresentar bastante perdas por inserção (50\%). As fibras HNLF são mais resistentes a deformações mecânicas e possuem em média $\gamma \sim 5 \mathrm{~W}^{-1} \mathrm{~km}^{-1}$, que para lasers com intensidades pequenas é necessário um comprimento de interação grande para satisfazer a condição de compressão. 
Modulador de fase Duas estruturas na forma de um carretel cortado longitudinalmente, levemente fora do eixo axial, são fixados cada um em uma extremidade de um bloco piezo-elétrico da THORLABS. A expansão máxima do bloco é de 4,6 $\mu \mathrm{m}$ quando submetido à tensão máxima, 150V. A fibra é então enrolada em torno da estrutura com um número de voltas suficiente para que o deslocamento do piezo possa varrer alguns modos da cavidade. As dimensões da nossa estrutura permitem que, com apenas quatro voltas de fibra, a cavidade consiga varrer alguns modos com o deslocamento do piezo.

Circulador óptico Utilizamos o circulador óptico da JDSU para fibra óptica. A perda por transmissão normal é de 1,48dB. O campo contrapropagante é rejeitado na transmissão normal em 40dB, e sofre uma atenuação na saída de monitoramento de 1,31dB. Alguns testes realizados mostraram que ele é insensível à polarização.

Controlador de polarização Trata-se de lâmina de onda para fibras. Esta consiste de loops de fibras em uma estrutura circular. O plano da estrutura pode ser girado em torno do eixo induzindo um retardamento de fase devido a uma deformação mecânica birrefringente [4]. Para uma fibra de raio $W$, o atraso de fase induzido pela deformação em um único loop de raio $R$ é

$$
\Delta \Psi=5,25 \frac{W^{2}}{R} .
$$

Acoplador Utilizamos acopladores comerciais conectorizáveis da THORLABS, com taxa de acoplamento 90/10. Como porta de entrada e saída de uma cavidade, suas perdas mais significativas são os conectores, somando mais de $1 \mathrm{~dB}$ em ambos, contra 0,64dB na inserção. Possui uma diferença na perda de inserção por polarização menor que $0,1 \mathrm{~dB}$, e uma estabilidade térmica menor que $0,3 \mathrm{~dB}$.

\subsection{Aparato Experimental}

A proposta experimental para a geração e deteç̧ão de estados comprimidos está representada pictoricamente na figura 5.7. A saída do laser de diodo é acoplado à cavidade de fibra através de um circulador óptico. Devido a atenuação sofrida pelo feixe do laser ao atravessar o circulador, a intensidade máxima na entrada $a$ do acoplador é de $16 \mathrm{~mW}$. Apenas $10 \%$ desta intensidade entra para a cavidade de fibra óptica. O ressoador em anel é constituído, em sua maior parte, por uma fibra óptica não birrefringente altamente não linear, com um fator de não linearidade 


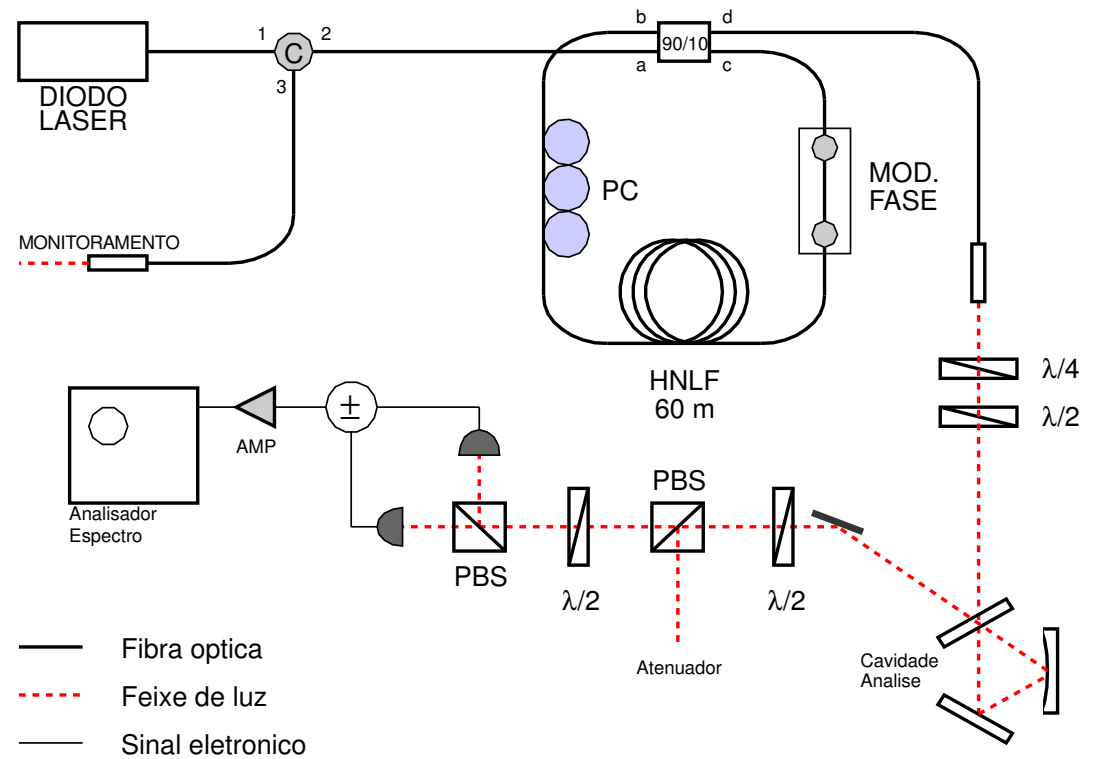

Figura 5.7: Proposta experimental para a geração e detecção de estados comprimidos, gerados através de um processo de 4WM em fibras ópticas. O feixe do laser de diodo é injetado no ressoador em anel de fibra óptica através de um acoplador comercial 90/10. Um sistema servo pode travar a cavidade em dessintonia nula, maximizando a intensidade do campo circulante e aumentando a eficiência na geração dos feixes gêmeos. Correlações de amplitude e fase entre pares de fótons podem ser investigadas através de um deslocamento de fase induzido pela cavidade de análise entre a portadora e as bandas laterais. As flutuações de intensidade e fase são transformadas em flutuações de corrente elétrica na saída do fotodiodo, e podem ser analisadas espalhando estas flutuações temporais no domínio da frequência pelo analisador de espectro.

de $6 \mathrm{~W}^{-1} \mathrm{~km}^{-1}$, e atenuação de $0,5 \mathrm{~dB} / \mathrm{km}$. O acoplador é fabricado com uma fibra óptica monomodo convencional, e possui alguns metros de comprimento. O comprimento total da cavidade é superior a $60 \mathrm{~m}$. Após a porta $c$ do acoplador, o acoplamento da fibra convencional com a fibra altamente não linear é estabelecido. A perda por inserção na conexão é cerca de $0,5 \mathrm{~dB}$, e é causada pelo desacordo de modo entre os dois modos fundamentais guiados pelas fibras.

A fibra é então enrolada em uma estrutura na forma de um carretel com raio de 1", cortado longitudinalmente, levemente deslocado do diâmetro. Fixado entre as duas paredes do carretel, um piezoelétrico de cerâmica atua sobre estes, alterando levemente a distância e expandindo a fibra enrolada. O número de voltas da fibra óptica sobre a estrutura não deve ser muito grande, 
caso contrário será induzida uma birrefringência pela deformação. Aplicada uma modulação sobre o piezo, reflete em uma modulação de fase do campo guiado, devido à oscilação no comprimento do caminho óptico.

A fibra de sessenta metros está enrolada em um cilindro, e este armazenado em uma caixa isolante para reduzir os efeitos causados pelas flutuações na temperatura. Depois, existe mais uma conexão de $0,5 \mathrm{~dB}$ acoplando a fibra não linear com a fibra convencional. Seguindo a conexão, a fibra passa através de três loops, cada um com raio de $33 \mathrm{~mm}$, constituindo como um todo, uma lâmina de $\frac{1}{2}$ onda. A fibra então volta ao acoplador pela porta $b$, fechando o loop da cavidade. As perdas estimadas em uma volta completa é de $1,03 \mathrm{~dB}$, contra 1,25dB medida.

A saída do ressoador de fibra pela porta $d$ é colimado por uma objetiva em um feixe propagante com cintura de aproximadamente $2 \mathrm{~mm}$. Duas lâminas de onda, $\frac{1}{4}$ e $\frac{1}{2}$, servem para corrigir qualquer efeito causado pela birrefringência induzida e possibilitar um controle manual sobre a polarização, respectivamente. Em seguida, o feixe de luz é acoplado à cavidade de análise em anel, feita com 2 espelhos planos e um curvo, com raio de curvatura de $50 \mathrm{~cm}$. Os espelhos são fabricados para serem altamente refletores em $1550 \mathrm{~nm}$ com incidência a $45^{\circ}$. O espelho plano de entrada possui reflexão de 0,99 , o segundo espelho plano de 0,995 , e o espelho curvo é suposto totalmente refletor. O perímetro da cavidade é medido em cerca de $34 \mathrm{~cm}$, permitindo estimar um FSR de $882 \mathrm{MHz}$. Com uma finesse de 337 medida, estimamos uma largura de linha de 2,6 MHz. Para o melhor funcionamento deste interferômetro, um acordo de modo rigoroso é necessário.

O caráter dispersivo da cavidade introduz uma fase relativa entre a fase da portadora e a fase dos modos vizinhos de ressonância da cavidade de fibra, tratados como bandas laterais. Para certos valores de dessintonia, flutuações de intensidade do campo refletido, na realidade, correspondem às flutuações de fase das bandas laterais. A portadora tem um papel análogo ao oscilador local na deteç̧ão homodina.

O feixe refletido pela cavidade de análise é composto pela oscilador local assim como muitos pares de modos do sinal e do complementar. Este feixe é então atenuado por um conjunto lâmina de meia onda mais PBS (Polarization Beam Splitter), a um nível de não saturação dos fotodiodos. Depois, o feixe atravessa novamente um conjunto idêntico ao anterior, desta vez para ser repartido igualmente e enviados a dois sistemas de detecção similares.

Cada detector é composto pelo fotodiodo ETX 300 da EPITAXX, mais uma eletrônica de amplificação de sinais elétricos de alta frequência. O sinal DC é medido por um osciloscópio com o propósito de ter um sinal de referência para uma possível normalização dos ruídos de alta frequência. O sinal HF é enviado a um divisor de tensão da Mini-Circuits, que realiza passivamente a operação de soma e a subtração dos sinais. Em seguida, o sinal resultante é amplificado novamente pelo amplificador eletrônico da SRS, modelo SR445, e medido pelo analisador de 
espectro da Agilent, modelo E4401B.

A grande dificuldade do experimento foi a estabilização e o travamento da cavidade de fibra. Começamos a testar cavidades com comprimentos de fibra óptica da ordem de km's de comprimento. Para estas cavidades, o FSR é da ordem de centenas de $\mathrm{kHz}$, isto é, começa a ser comparável às vibrações mecânicas $(\sim 500 \mathrm{~Hz})$, tornando impraticável a sua estabilização. A própria flutuação do laser em frequência é o suficiente para varrer milhares de modos da cavidade. O primeiro sinal de uma cavidade funcional foi obtido para uma fibra de $300 \mathrm{~m}$. No

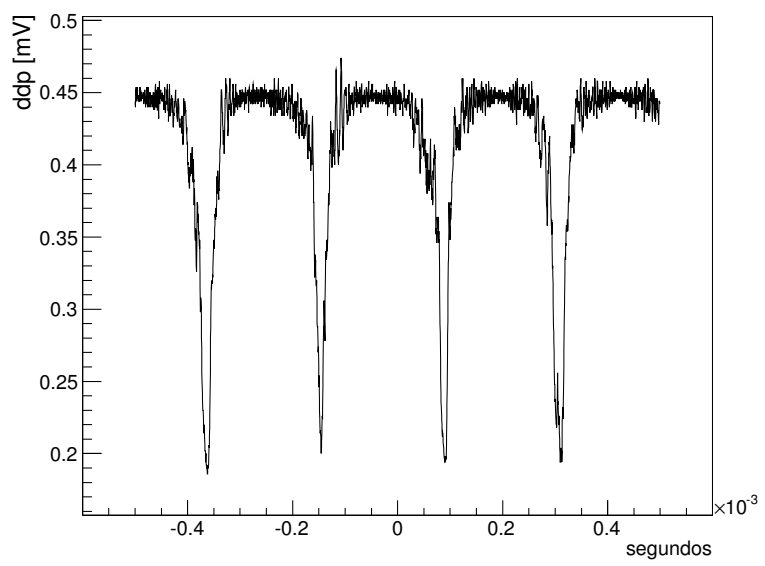

Figura 5.8: Comportamento de uma cavidade de 300m com o laser RIO.

entanto, flutuações térmicas causam alterações aleatórias no comprimento total da fibra. Para variações de temperatura da ordem de mili graus Celsius, a alteração no comprimento da fibra é o suficiente para alcançar um modo da cavidade.

Em uma cavidade de $60 \mathrm{~m}$, temos estabilidade suficiente para implementar medidas. Se uma modulação fraca de $\mathrm{kHz}$ é aplicada ao piezoelétrico, a oscilação na intensidade do campo de bombeio pode ser observada no sinal DC medido pelo fotodiodo. O sistema de travamento baseado na geração de um sinal de erro a partir de uma modulação dos picos de Airy da cavidade, conhecido como dither, juntamente com um sistema de travamento (Servo System), pode ser utilizado para travar a cavidade de fibra em uma dessintonia de interesse. O funcionamento básico de um sistema de travamento pode ser verificado no anexo A.

\subsection{Primeiras análises}

Na seção 4.4, obtivemos o limiar de oscilação dos feixes gêmeos (4.4.12), decorrente de um processo não linear $\chi^{(3)}$, que ocorre dentro de uma cavidade em anel de fibra óptica. Mediante 
o formalismo de entrada e saída de cavidades [55], podemos obter o limiar de oscilação do feixe de bombeio do lado de fora da cavidade, na entrada $a$

$$
P_{\text {limiar }}^{a}=\frac{\left(2-\eta-\eta_{r}\right)^{3}}{8 \kappa(1-\eta) L}
$$

onde, 1- $\eta$ é o acoplamento de entrada da cavidade, $1-\eta_{r}$ representa as perdas incontroláveis em uma volta completa, $L$ é o comprimento da fibra e $\kappa$, seu fator de não linearidade. Nesta cálculo, consideramos que a cavidade tem a mesma resposta para todos os modos. A representação gráfica deste valor em função do comprimento da cavidade pode ser observada na figura 5.9. Em uma cavidade de $60 \mathrm{~m}$ com fator de não linearidade $\kappa=6 \mathrm{~W}^{-1} \mathrm{~km}^{-1}$, obtemos um limiar

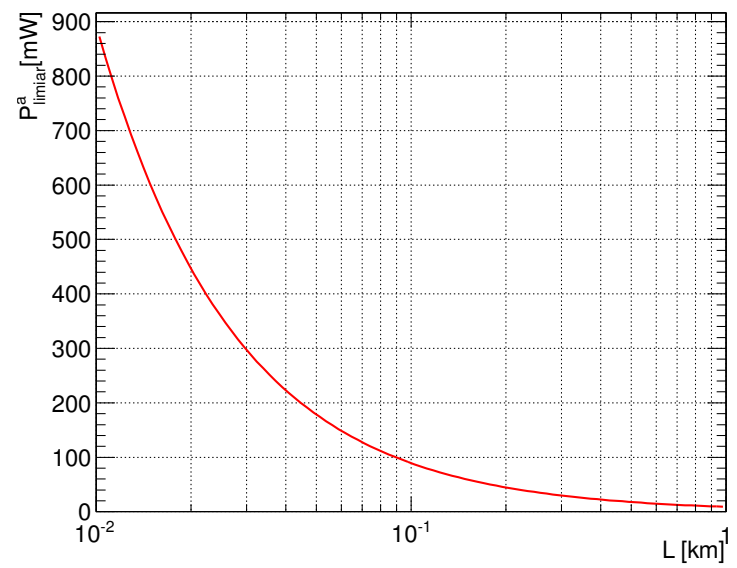

Figura 5.9: Limiar de oscilação dos feixes gêmeos para uma cavidade em anel de fibras. O campo aprisionado sofre efeitos não lineares do tipo Kerr. Para uma cavidade de $60 \mathrm{~m}$, o ganho supera as perdas para um feixe de bombeio de $150 \mathrm{~mW}$, iniciando a geração dos feixes gêmeos.

de oscilação de $150 \mathrm{~mW}$. O limiar de oscilação do espalhamento Brillouin para uma fibra com as mesmas especificações é de $133 \mathrm{~mW}$, sem cavidade. Portanto, com esta fibra não é possível conseguir a geração dos feixes gêmeos sem antes iniciar a geração estimulada do espalhamento Brillouin. No artigo [43], os autores propõem a construção de um isolador óptico que reduz significativamente o espalhamento Stokes a um nível em que seja possível medir compressão nas quadraturas acima do limiar de Brillouin. 


\subsubsection{Efeito cavidade}

O sistema de travamento permite estabilizar o ressoador de fibra em duas condições de dessintonia. Em ressonância, a reflexão pela cavidade é mínima e o campo circulante atinge o seu maior valor de intensidade. Fora de ressonância, a cavidade é travada no máximo de reflexão e a intensidade do campo circulante é mínima. Um efeito interessante acontece quando travamos a cavidade fora de ressonância. Logo que o sistema de travamento é ativado, e a cavidade é travada no máximo, observamos no analisador de espectro o sinal resultante da soma dos ruídos de amplitude. O gráfico representativo do sinal está exposto na figura 5.10. Inúmeros
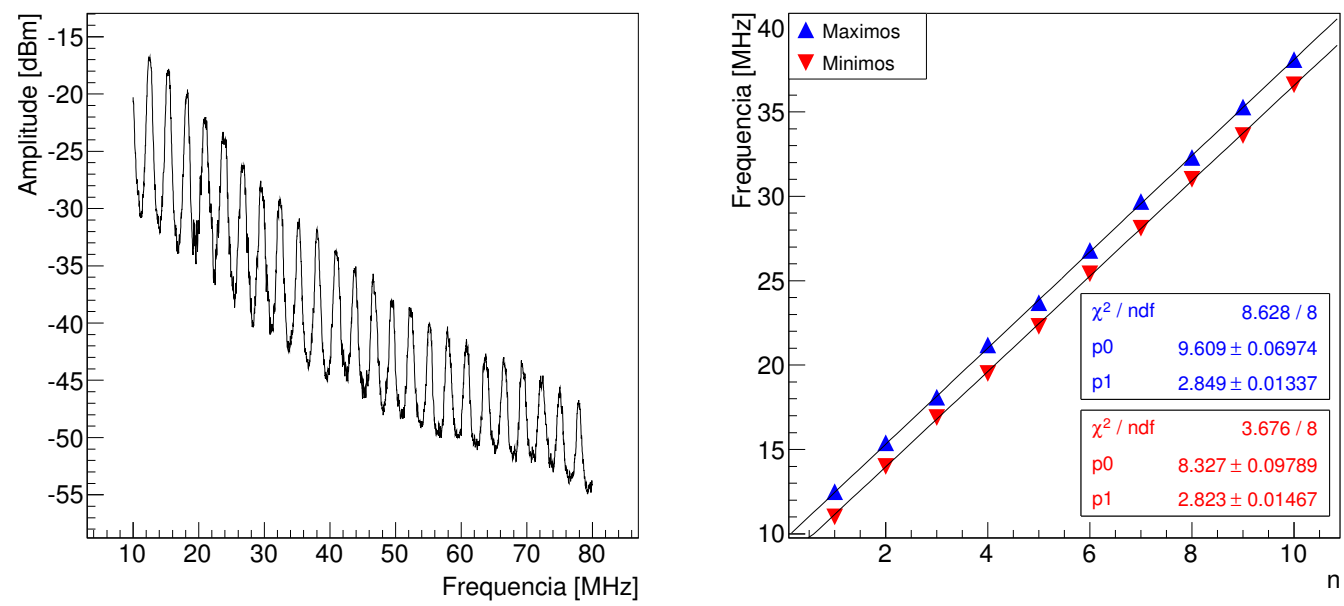

Figura 5.10: O gráfico da esquerda mostra o sinal observado no analisador de espectro quando medimos a soma dos ruídos de intensidade, na situação de máxima dessintonia. O sinal é observado somente por alguns instantes quando bloqueamos o campo circulante dentro da cavidade de análise. O gráfico da direita mostra as curvas dos valores de máximos e mínimos observados. Obtemos que os máximos correspondem a valores semi-inteiros do FSR, enquanto que os mínimos correspondem a valores inteiros do FSR.

máximos e mínimos são observados, com frequências indo de 0 até o limite do sistema de deteç̧ão, em 200MHz. Os valores de máximos do gráfico estão todos distanciados entre si por uma frequência de $2,849 \pm 0,005 \mathrm{MHz}$, enquanto que os valores de mínimos estão distanciados de $2,823 \pm 0,005 \mathrm{MHz}$

As condições de travamento da cavidade quando observamos o efeito, e quando ele desaparece, pode ser observado na figura 5.11. A curva em preto representa os vales de reflexão da cavidade e as flutuações em azul indicam o travamento da cavidade em ressonância. Com a altura máxima destas flutuações podemos estimar os desvios da dessintonia no mínimo. As duas retas em vermelho nos gráficos mostram a cavidade travada fora de sintonia. No gráfico da esquerda, 
o efeito de máximos e mínimos é observado. O tempo de travamento nesta condição é muito curto, e o estado da cavidade passa rapidamente para a condição representada no gráfico da direita.

A primeira reação foi relacionar o perfil da curva com o efeito da cavidade sobre o ruído. Uma vez que não conhecemos o tamanho da cavidade com grande precisão, os espaçamentos entre cada máximo ou mínimo seria a assinatura da cavidade, pois estes valores são compatíveis com o intervalo espectral livre. Notando o comportamento linear dos máximos e mínimos, percebemos que os máximos ocorrem sempre em múltiplos de meio intervalo espectral livre, enquanto que os mínimos estão localizados em intervalos inteiros do FSR. Observando o travamento da cavidade de fibra na figura 5.11, temos que o efeito surge quando a portadora está sintonizada exatamente entre duas ressonâncias. No entanto, as bandas laterais estão entrando e sendo

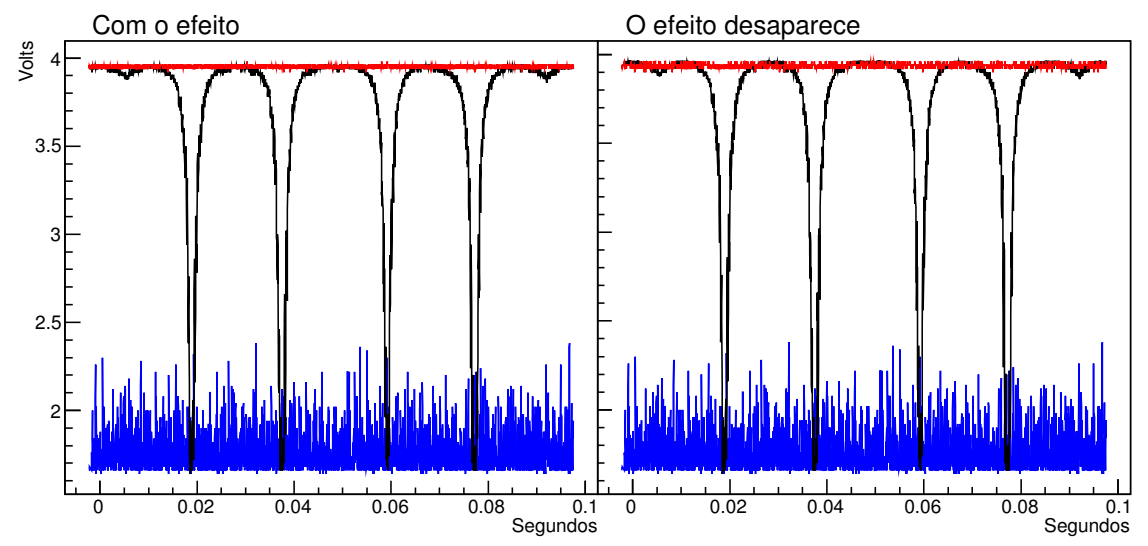

Figura 5.11: Gráficos mostrando as duas condições de travamento, fora de ressonância, em vermelho, e em ressonância, em azul. Por um instante curto de tempo, um efeito interessante acontece quando a cavidade encontra-se bem no máximo da dessintonia.

processadas pela cavidade a cada semi-múltiplo $(n+1 / 2)$ do intervalo espectral livre. Nesta condição, a cavidade de fibra atua como uma cavidade de análise, impondo uma defasagem de $\pi$ entre a portadora e as bandas laterais, o resultado será uma meia rotação de elipse de ruído naquela frequência específica $(n+1 / 2) F S R$, revelando o ruído de fase. Os mínimos correspondem aos pontos em que os modos das bandas laterais, assim como a portadora, não entram na cavidade, e são diretamente refletidos, mantendo seu caráter original. Todo o comportamento é linear, e é um interessante artifício da cavidade em fibra óptica com um FSR pequeno.

Infelizmente, o sistema de travamento não consegue manter a cavidade nesta condição por muito tempo. Após alguns segundos, a cavidade ainda é mantida fora de ressonância, mas a um nível um pouco inferior. A figura 5.12 mostra um tratamento estatístico do travamento da cavidade. Em média, o travamento em ressonância é dado com uma dessintonia de $\Delta=0,22$, em uni- 

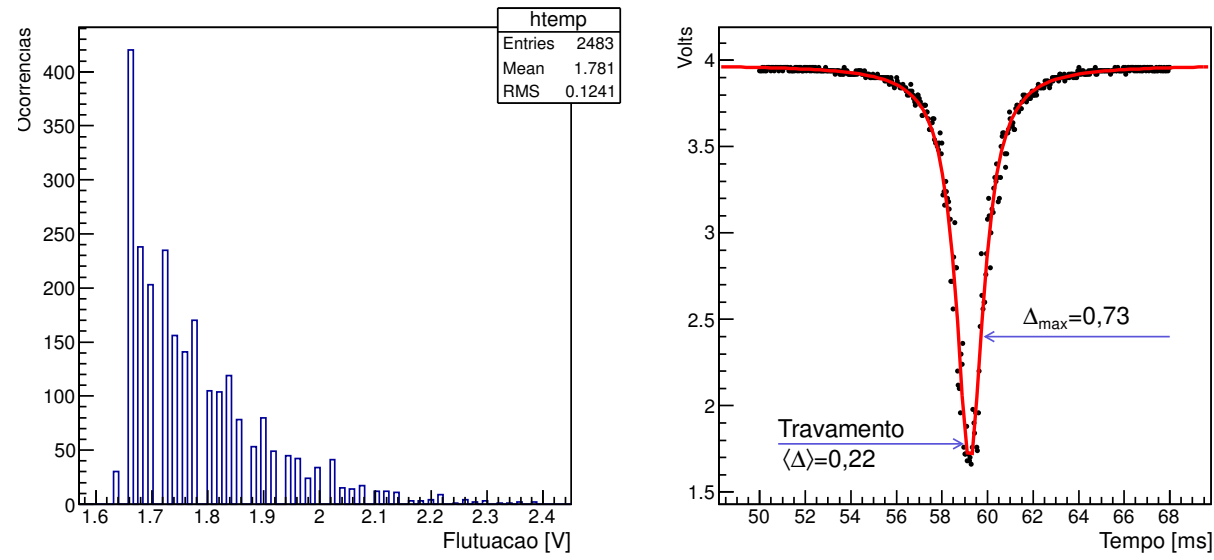

Figura 5.12: Tratamento estatístico da dessintonia do travamento da cavidade. O gráfico da esquerda é um histograma da flutuação do campo intracavidade quando está em ressonância com a cavidade. O valor médio de dessintonia com a cavidade em ressonância é de $\Delta=0,22$, com flutuação máxima levando a valores de até $\Delta=0,73$.

dades de largura de linha da cavidade. A máxima flutuação da cavidade em ressonância é de $\Delta=0,73$.

\subsection{Medidas de ruído}

Com a cavidade de análise funcionando, as primeiras medidas de ruído realizadas foram aquelas da caracterização do ruído do laser. Evidenciamos este ruído, sem a cavidade de fibra, injetando o laser diretamente na cavidade de análise e fazendo as operações de soma e diferença dos campos, ver figura 5.5. Observamos que, o ruído de intensidade do laser está ao mesmo nível do shot-noise, apresentando pouco ruído clássico. Em contraste, um excesso de ruído de fase do laser é verificado em todo o intervalo de frequência mensurável, típico de um laser de diodo.

É importante perceber também que, entre $80 \mathrm{MHz}$ e $140 \mathrm{MHz}$, o ruído eletrônico apresenta um comportamento diferente daquele mostrado para frequências menores. Flutuações de grande amplitude em múltiplas frequências podem comprometer qualquer análise futura feita neste intervalo. Apesar de existir um intervalo entre $140 \mathrm{MHz}$ e $170 \mathrm{MHz}$, onde o ruído eletrônico não apresenta grandes variações, o nível do shot-noise está a menos de $3 \mathrm{~dB}$ do eletrônico, limitando qualquer observação neste intervalo. Portanto, temos um intervalo de confiança garantido pelo sistema de detecção de $10 \mathrm{MHz}$ à $80 \mathrm{MHz}$. 
O fato de haver de início um excesso de ruído intrínseco ao laser, de aproximadamente $20 \mathrm{~dB}$, compromete qualquer expectativa de medir uma compressão nesta quadratura abaixo do limite quântico. Qualquer observação feita sobre esta quadratura será no sentido comparativo.

Com o aparato experimental funcionando, comparamos a influência da cavidade sobre os ruídos de quadraturas nas duas configurações da cavidade, fora de ressonância e em ressonância. Os resultados obtidos podem ser vistos na figura 5.13. As medidas foram realizadas com duas
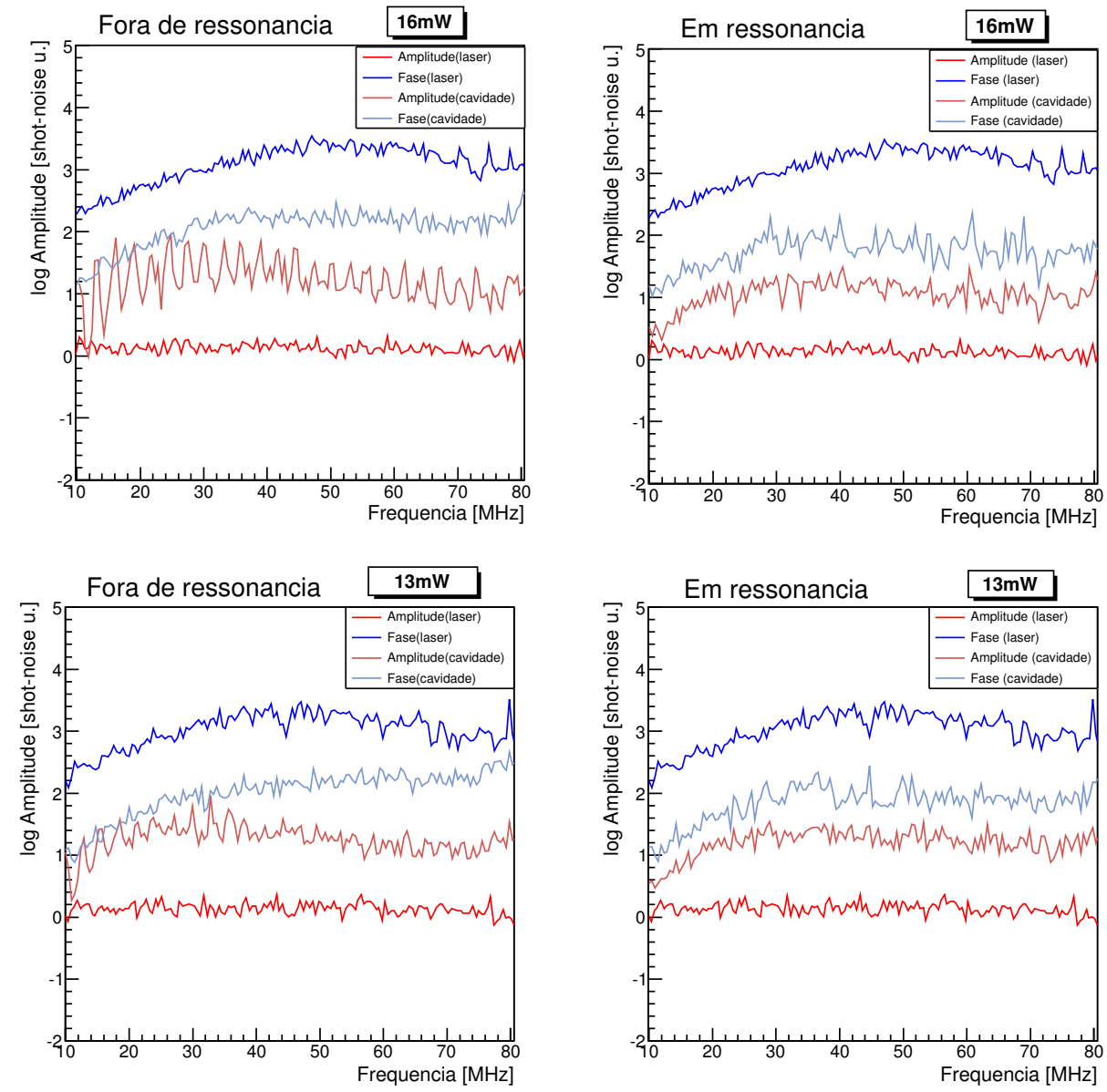

Figura 5.13: Gráficos comparativos entre os ruídos de amplitude e fase do laser, sem e sob a atuação da cavidade em duas situações de dessintonia, longe da ressonância e em ressonância. Medimos para duas potências de bombeio, $16 \mathrm{~mW}$ e $13 \mathrm{~mW}$. Apesar da cavidade fora de ressonância não atuar sobre o ruído da portadora, ela ainda pode atuar sobre as bandas laterias da mesma.

potências do campo de bombeio na entrada do ressoador, $16 \mathrm{~mW}$ e $13 \mathrm{~mW}$. Os valores de intensidade são alterados usando atenuadores ópticos de $1 \mathrm{~dB}$ para fibras. Nos limitamos a estes valores porque atenuações superiores a estas, o sinal ótico nos detectores se aproxima do ruído 
de fundo.

Observamos que nas duas condições de dessintonia, no intervalo de frequências que vai de $10 \mathrm{MHz}$ à $80 \mathrm{MHz}$, a cavidade atua no sentido de reduzir o ruído de fase do laser às custas da amplificação no ruído de amplitude. O interessante é que mesmo fora da ressonância, a cavidade induz uma redução no ruído de fase, quando esperaríamos que nesta condição, ela atuasse como um objeto passivo, sem alterar significativamente o ruído.

A figura 5.14 mostra também uma análise comparativa entre a atuação da cavidade longe da ressonância e em ressonânica. Observamos que a atuação da cavidade em ressonância é ligeiramente mais significativa do que a cavidade fora de ressonância. As medidas de rotação de
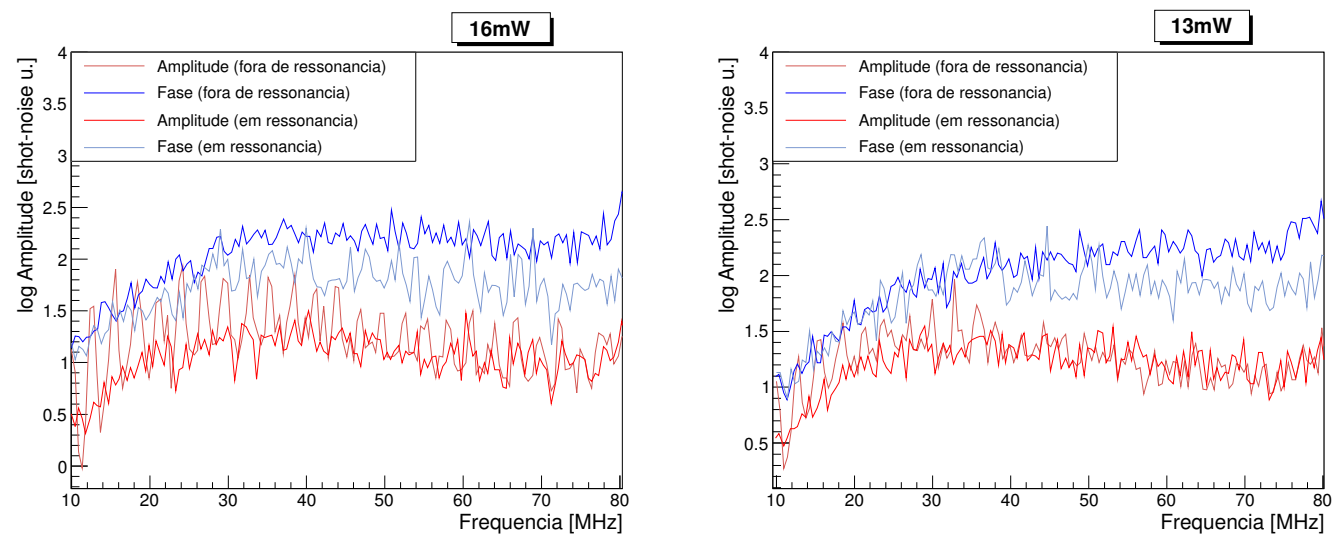

Figura 5.14: Gráficos comparativos mostrando a diferença de atuação da cavidade fora de ressonância e em ressonância. Em ressonância, devido à presença da portadora no ressoador, o processo que leva à redução de ruído de fase é mais significativo. As medidas foram realizadas com uma potência de bombeio na entrada da cavidade de $16 \mathrm{~mW}$ e $13 \mathrm{~mW}$.

elipse foram realizadas para frequências inferiores a $20 \mathrm{MHz}$, pois sabemos da literatura que não há picos de GAWBS até esta frequência. Apesar da liberdade de podermos realizar medidas em qualquer frequência, escolhemos aquelas que apresentam um máximo no ruído de amplitude dado pelo efeito da cavidade, ver figura 5.10. Contudo, desta vez com a cavidade em ressonância, encontramos um excesso de ruído nas quadraturas de fase com relação ao ruído da cavidade fora de ressonância. O resultado pode ser visto na figura 5.15 .

Infelizmente, o excesso de ruído de fase do laser impediu qualquer estudo mais detalhado da atuação da cavidade como um meio de intensificar os processos não lineares. Apesar da observação direta de uma redução do ruído de fase às custas de um aumento no ruído de amplitude, não é permitido afirmar isto está acontecendo devido à não linearidade da fibra. Qualquer afirmação neste sentido é necessário um laser com pouco ruído em ambas as quadraturas, e a realização de diversas medidas de ruído para diferentes potências de bombeio. Entretanto, para 


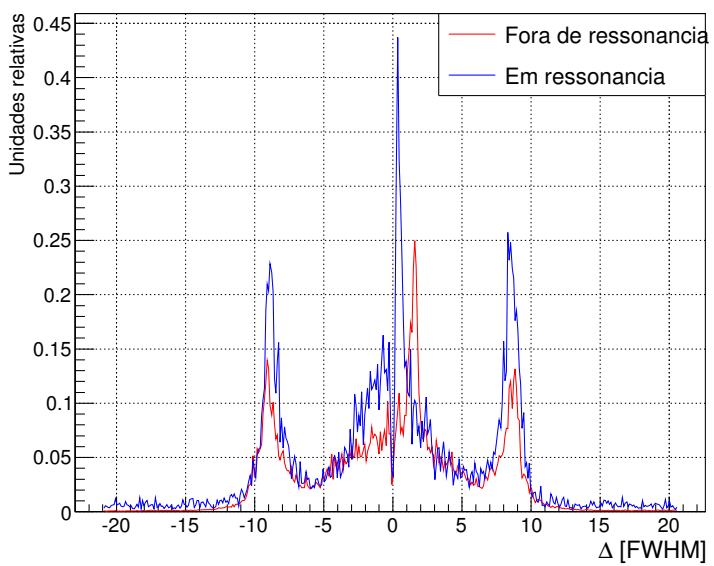

Figura 5.15: Medida de rotação de elipse do campo, na frequência de 19,6MHz. Ambas as curvas estão normalizadas pelo sinal DC, e o eixo da abscissa representa a dessintonia da cavidade de análise em unidades de largura de linha.

a visualização de uma redução de ruído abaixo do limite quântico, é necessário realizar medidas de ruído para frequências específicas, entre dois picos de GAWBS. Portanto a caracterização do espectro de GAWBS é necessária, e para isso, precisamos também de um perfil espectral, intrínseco do laser, com pouco ruído, o excesso de ruído de fase do laser pode encobrir qualquer determinação do perfil do espectro de GAWBS. 


\section{Conclusões e perspectivas}

Neste trabalho, através de um desenvolvimento teórico do processo de $4 \mathrm{WM}$ que ocorre em uma fibra óptica na geometria de cavidade, mostramos teóricamente que é possível gerar estados comprimidos da luz abaixo e acima do limiar de oscilação. Uma vez atingido o limiar de geração dos feixes gêmeos, múltiplos modos distanciados de um intervalo espectral livre podem estar correlacionados. Abaixo do limiar, encontramos uma dependência do espectro de compressão com a potência do campo de bombeio. Encontramos a máxima compressão nas quadraturas quando atingimos o limiar de oscilação dos feixes gêmeos, neste caso, as solução para os campos sinal e complementar se tornam biestáveis.

Dois fatores impediram uma investigação detalhada destas situações. O primeiro, uma limitação física, não podemos alcançar o limiar de geração dos campos sem antes atingir o limiar de oscilação do espalhamento Brillouin estimulado. A segunda limitação é puramente técnica, não tínhamos um laser em 1550nm capaz de fornecer a intensidade necessária para entrarmos neste regime.

Através de uma caracterização detalhada do espectro de GAWBS, é possível determinar janelas de frequências onde a queda entre dois picos Brillouin atinja o nível de shot-noise. Estas são regiões promissoras para a procura de compressão nas quadraturas.

Por fim, descrevemos o aparato experimental necessário para a produção e medição de estados comprimidos. Infelizmente, devido ao excesso de ruído de fase intrínseco aos lasers de diodo, não pudemos medir uma redução de ruído desta quadratura a níveis abaixo do limite quântico. No entanto, observamos que o efeito da cavidade sobre os ruídos de amplitude e fase dos modos de vácuo, atua no sentido de reduzir o ruído de fase como consequência de um aumento no ruído de amplitude, comportamento previsto para um processo de compressão condicionado ao limite da incerteza.

Ao longo deste trabalho, testamos e estudamos o comportamento de diversos lasers para ser utilizado como fonte de luz coerente em um experimento envolvendo uma cavidade de centenas ou dezenas de metros. Grande parte deste período esteve ligado à construção e estabilidade 
da cavidade. Testamos diversos modelos e comprimentos de fibras para escolher a que melhor se adaptasse à proposta experimental, e que dentre os lasers disponíveis mostrasse uma estabilidade suficiente para realizar medidas em um intervalo de tempo razoável. Contudo, o fator decisivo foi o excesso de ruído de fase do laser, impedindo qualquer análise ao nível quântico do comportamento dos ruídos.

Este trabalho serviu como base para uma familiarização com processos não lineares que ocorrem em guias de ondas, como as fibras ópticas. Os resultados serviram para uma preparação de investigações futuras em sistema integrados. Experimentos recentes no grupo da profa. Michal Lipson, na Univ. de Cornell demonstraram a construção de osciladores paramétricos em chips de silício, compátiveis com a tecnologia de construção CMOS empregada nos dispositivos semicondutores [29]. Desta vez, para uma cavidade com perímetro da ordem de dezenas de micrômetros, teremos cavidades em anel com um FSR da ordem de $100 \mathrm{GHz}$, com fator de qualidade altíssimo $Q \sim 500000$. Nesta situação, potências pequenas do feixe de bombeio acoplados com a cavidade são capazes de estabelecer intensidades muito altas para o campo propagante dentro da cavidade.

a
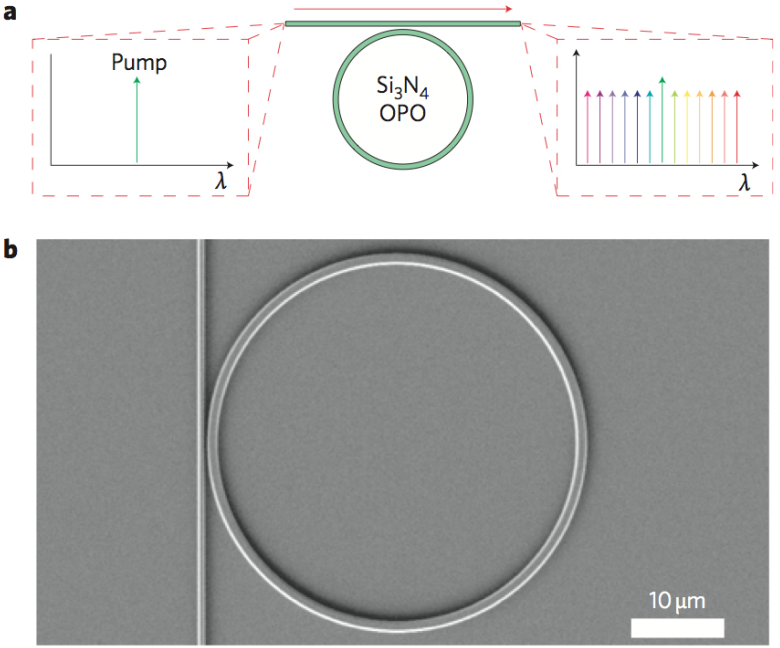

Figura 6.16: Em a), mostra uma presentação da geração de múltiplos modos de oscilação, distanciados de um FSR. Em b), temos uma foto da microcavidade em anel, também capaz de impor interações não lineares entre a radiação e a matéria. Figuras extraídas da referência [29]. 


\section{Apêndice A}

\section{Sistema de travamento}

Neste anexo irei tratar superficialmente a idéia por trás do sistema de travamento, cuja aplicação se faz necessária a qualquer experimento que exija uma configuração estável do sistema. Para que se consiga estabilizar a configuração de um experimento, devemos ser capazes de estabilizar seus parâmetros envolvidos. A estabilização é feita por um processo interativo, no qual um ciclo de medida e realimentação é repetido constantemente.

O sistema de travamento, conhecido como dither $\&$ locking, nos permite travar o sistema em um pico de ressonância, quer este seja um máximo ou um mínimo. Isso é feito medindo a derivada do sinal de saída do experimento. Tal medida nos permite conhecer o quão longe estamos do ponto desejado, isto é, de derivada nula. O sistema então gera um sinal elétrico que é proporcional ao desvio da derivada com relação ao zero. Este sinal é responsável direto pelo processo de realimentação. Qualquer perturbação externa sobre o parâmetro do experimento, haverá um sinal elétrico contrário ao sentido da perturbação gerado pelo sistema de travamento, tal processo é conhecido como realimentação negativa.

O processo de geração do sinal de erro baseia-se na análise da alteração do sinal de saída do fotodetector como consequência de uma modulação realizada em um parâmetro do experimento. Partindo da consideração de que o experimento tenha uma resposta linear à modulação, a alteração na intensidade de luz sobre o fotodetector será proporcional à derivada do pico de ressonância. Portanto, a partir da medida feita da mudança da amplitude no fotodetector, devido à modulação, conseguimos saber em que ponto do pico de ressonância o sistema se encontra, este processo é conhecido como dithering. A figura (A.1) mostra um diagrama de blocos do sistema de travamento. O gerador de sinal fornece um sinal senoidal que irá modular o parâmetro do experimento, este parâmetro pode ser a frequência do laser ou a tensão sobre o PZT (piezoeletric-actuator). O sinal modulado de saída do experimento é enviado para um 


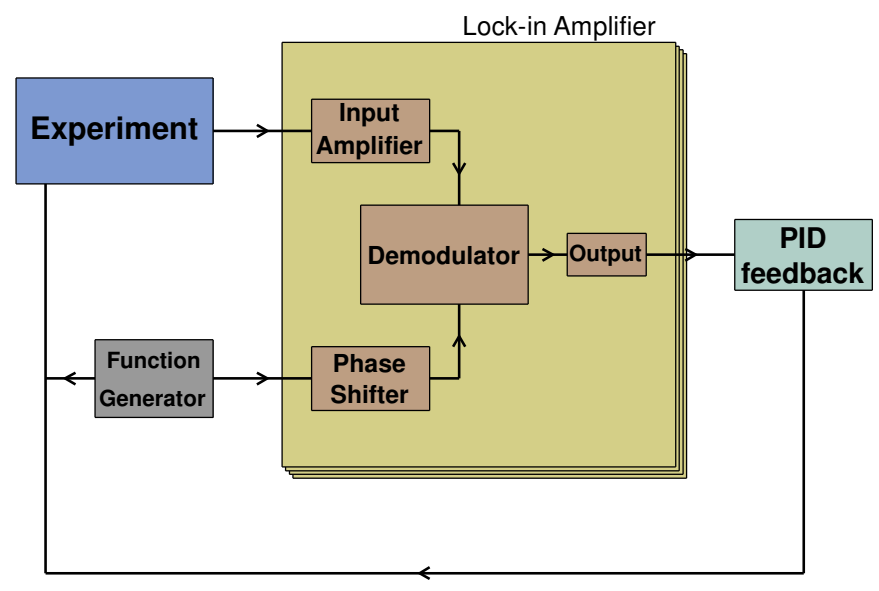

Figura A.1: Diagrama de blocos do sistema de travamento

amplificador de ganho variável. Simultaneamente, um sinal de referência de onda quadrada é enviado do gerador de sinal para o controlador de fase. O controlador de fase corrige a diferença de fase entre o sinal do experimento e a referência, que pode ser causada pela diferença de percurso entre os dois sinais.

Em seguida ambos os sinais são enviados ao demodulador. O demodulador gera um sinal DC cuja amplitude é proporcional ao valor da derivada do sinal. Posteriormente o sinal é amplificado na saída.

O processo de geração do sinal de erro ocorre no bloco conhecido como Lock-in Amplifier. Estes são usados para medir com bastante acurácia a amplitude de um sinal $A C$ que pode estar imerso em ruído ou como também medir a derivada do sinal de entrada.

Freqüentemente desejamos medir a amplitude de um sinal que é medido por um detector. Se o sinal tiver uma intensidade pequena, a relação sinal-ruído pode ser pobre, dificultando uma medida precisa da amplitude. O que o lock-in faz é medir a amplitude da componente de Fourier em determinada frequência de referência do sinal ruidoso. A frequência de referência é determinada pelo experimento, que pode ser definida pelo gerador de função que atua sobre um oscilador.

Suponha que queiramos medir um sinal senoidal na frequência de referência $\omega_{s}$ definida pelo oscilador. O lock-in gera seu próprio sinal senoidal de frequência $\omega_{L}$ e faz a multiplicação dos dois sinais.

$$
V_{\text {out }}=V_{s} V_{L} \sin \left(\omega_{s} t-\theta_{s}\right) \sin \left(\omega_{L} t-\theta_{L}\right),
$$


onde $V_{s}$ e $V_{L}$ são as amplitudes dos sinais de referência e do lock-in respectivamente. O resultado final são dois sinais $A C$, um na frequência da diferença $\omega_{s}-\omega_{L}$ e outro na frequência da soma $\omega_{s}+\omega_{L}$. Em seguida, a saída $V_{\text {out }}$ passa por um filtro passa baixa e o sinal $A C$ é filtrado. Em geral não sobraria nada, a menos que a frequência do Lock-in seja a mesma que a frequência do sinal. Neste caso temos que o argumento do co-seno é zero, e como consequencia temos um sinal $D C$ proporcional à amplitude do sinal na frequência de referência

$$
V_{\text {out }}=1 / 2 V_{s} V_{L} \cos \left(\theta_{s}-\theta_{L}\right)
$$

O fato importante é que o lock-in mede a contribuição de cada frequência no espaço de Fourier sobre o sinal de saída do experimento, bastando escolher a frequência desejada na referência do lock-in. Por exemplo, para uma onda quadrada de amplitude pico a pico de 2, podemos escrever sua decomposição no espaço de Fourier como 1, $27 \sin (\omega t)+0,42 \sin (3 \omega t)+0,25 \sin (5 \omega t)+\ldots$. Portanto, podemos ter acesso a cada amplitude da decomposição, devemos apenas escolher a frequência desejada na referência do lock-in. A precisão na medida da contribuição de cada frequência depende do ajuste da constante de tempo do lock-in, que está relacionado com a largura do filtro passa baixa.

À essa capacidade de realizar medidas de amplitude em determinadas frequências nos leva a uma técnica de importância fundamental no esquema de travamento: a medição da derivada do sinal. A idéia é modular com uma senóide de frequência $\Omega$ o oscilador do experimento, produzindo um sinal de saída $V(t)=V(x) \approx V\left[x_{\text {center }}+\Delta x \cos (\Omega t)\right]$, onde $x(t)$ é o parâmetro controlado pelo oscilador.

O lock-in amplifier com frequência de referência $\Omega$ produzirá então um sinal, definido como sinal de erro $\epsilon(t)$, que é a componente de Fourier de $V(t)$ na frequência $\Omega$. Expandindo $V(x)$ em série de Taylor, para um sinal de modulação pequeno, temos

$$
V(x)=V\left[x_{\text {center }}+\Delta x \cos (\Omega t)\right] \approx V\left(x_{\text {center }}\right)+\partial_{x} V\left(x_{\text {center }}\right) \cdot \Delta x \cos (\Omega t)+\ldots
$$

e a componente de Fourier é

$$
\epsilon(x) \sim \Delta x \cdot \partial_{x} V(x) .
$$

Independente da forma de $V(t)$, selecionando a componente de Fourier do sinal modulado produz um sinal de erro proporcional à derivada $\partial_{x} V(x)$.

No processo de travamento da cavidade ao laser, o oscilador pode ser tanto o laser, no qual é feito a modulação na frequência do mesmo, ou quanto o PZT, onde a modulação é feita no comprimento óptico da cavidade.

A figura abaixo exemplifica com dois gráficos o processo de estabilização do sistema. O primeiro 
mostra o pico de reflexão da cavidade com uma modulação de frequência $\Omega$ atuando sobre o $P Z T$, enquanto que o segundo gráfico mostra o sinal de erro gerado pelo Lock-in Amplifier quando oscila com a mesma frequência da modulação. O sinal de erro possuí propriedades
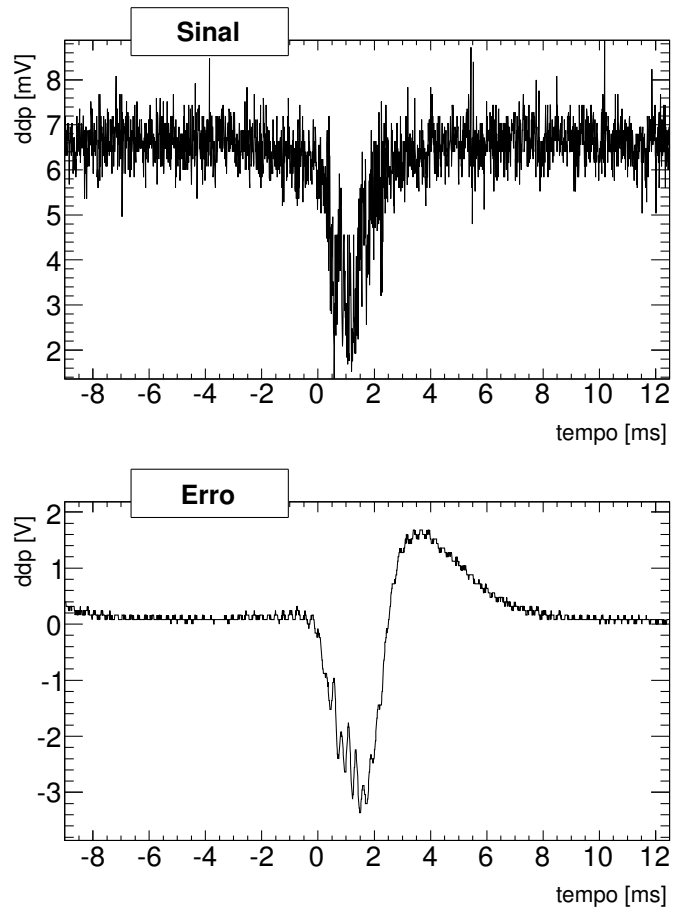

Figura A.2: O primeiro gráfico representa o pico de ressonância da cavidade. O segundo gráfico mostra o sinal de erro gerado pelo Lock-in Amplifier, sintonizado na frequência de referência da modulação.

desejadas de que $\epsilon\left(x_{0}\right)=0$ e $d \epsilon / d x\left(x_{0}\right) \neq 0$; então ele pode ser usado em um processo de realimentação para travar o sistema no pico de ressonância.

O sinal de erro está pronto para ser lido pelo PID, cujo propósito é determinar a distância da configuração do sistema ao estado desejado. O PID gera um sinal elétrico de correção $u(\epsilon)$ que irá realimentar o oscilador na direção de estabilizar o sistema. A eletrônica do PID executa três operações sobre o sinal de erro: proporcionalidade, integração e diferenciação;

$$
u(\epsilon, t)=g_{p} \epsilon(t)+g_{i} \int_{0}^{\tau} \epsilon(t) d t+g_{d} \frac{d \epsilon(t)}{d t} .
$$

Onde $g_{p}, g_{i}$ e $g_{d}$ são ganhos ajustáveis das operações de proporcionalidade, integração e diferenciação respectivamente. Sem o sistema de realimentação do PID, o estado do sistema não evoluirá ou ficará à disposição de flutuações externas. 
Seja $x_{0}$ o estado atual do oscilador a ser controlado, com o PID ligado, o estado evoluirá segundo a correção

$$
x(t+\Delta t)=x_{0}+u(\epsilon, t)
$$

Se considerarmos uma resposta temporal rápida $r_{t}$ do sistema devido à modulação, podemos escrever a equação (A.0.6) como

$$
x(t)+r_{t} \frac{x(t)}{d t}=x_{0}+g_{p} \epsilon(t)+g_{i} \int_{0}^{\tau} \epsilon(t) d t+g_{d} \frac{d \epsilon(t)}{d t} .
$$

Normalmente, no processo de realimentação, não utiliza-se a operação de derivação. Esta operação aumenta a velocidade de estabilização do sistema, mas geralmente é desnecessária. Outra razão para desligar esta operação é devido à dificuldade de controla-lá na prática, devido à sensibilidade do sistema ao processo [60]. Com a operação de derivação desligada e escrevendo $\epsilon(t)=x(t)-x_{d}$ a distância do estado atual ao estado desejado, podemos derivar a equação (A.0.7) e escreve-lá na forma

$$
r_{t} \frac{d^{2} x(t)}{d t^{2}}+\left(1-g_{p}\right) \frac{d x(t)}{d t}-g_{i} x(t)=-g_{i} x_{d},
$$

cuja solução por verificação direta é

$$
x(t)=A \exp \left(b_{+} t\right)+B \exp \left(b_{-} t\right)+x_{d},
$$

com $b_{ \pm}$dado por

$$
b_{ \pm}=\frac{\left(g_{p}-1\right) \pm \sqrt{\left(g_{p}-1\right)^{2}+4 g_{i} r_{t}}}{2 r_{t}} .
$$

As constantes $A$ e $B$ são obtidas pelas condições iniciais. O importante é que no caso de realimentação negativa, isto é $b \pm$ negativo, as exponenciais vão à zero e a variável do oscilador converge para o valor de desejado. 



\section{Bibliografia}

[1] rio Orion Laser Module. http://rio-inc.com/pdf/Rio_Orion_Product_Brief_Update_WEB.pdf.

[2] Govind P. Agrawal. Nonlienar Fiber Optics. ACADEMIC PRESS, 2001.

[3] Hans-A. Bachor and Timothy C. Ralph. A Guide to Experiments in Quantum Optics. WILEY-VCH, 2004.

[4] Ralph A Bergh, Herbert C Lefevre, and Herbert J Shaw. Single-mode fiber-optic polarizer. Optics letters, 5(11):479-481, 1980.

[5] Roy S. Bondurant, Prem Kumar, Jeffrey H. Shapiro, and Mari Maeda. Degenerate fourwave mixing as a possible source of squeezed-state light. Phys. Rev. A, 30:343-353, Jul 1984.

[6] RW Boyd. Nonlinear Optics. 2003.

[7] Claude Cohen-Tannoudji, Bernard Diu, and Frank Laloë. Quantum mechanics, 2 volume set, 2006.

[8] MJ Collet and DF Walls. Squeezing spectra for nonlinear optical systems. Phys. Rev. A, $32: 2887,1985$.

[9] G Mauro D'Ariano, Matteo GA Paris, and Massimiliano F Sacchi. Quantum tomography. Advances in Imaging and Electron Physics, 128:205-308, 2003.

[10] A. F. R. de Toledo Piza. Mecânica Quântica. Editora da Universidade de São Paulo, 2003.

[11] P.D. Drummond, K.J. McNeil, and D.F. Walls. Non-equilibrium transitions in sub/second harmonic generation. Optica Acta: International Journal of Optics, 27(3):321-335, 1980.

[12] Claude Fabre. Quantum fluctuations in light beams. 4 place Jussieu, F75252 Paris Cedex 05, France. 
[13] Claude Fabre, E Giacobino, A Heidmann, L Lugiato, S Reynaud, M Vadacchino, and Wang Kaige. Squeezing in detuned degenerate optical parametric oscillators. Quantum Optics: Journal of the European Optical Society Part B, 2(2):159, 1990.

[14] William Feller. An introduction to the theory of probability and its applications. Mir, Moscow, 1967.

[15] C. Gardiner and P. Zoller. Quantum Noise: A Handbook of Markovian and Non-Markovian Quantum Stochastic Methods with Applications to Quantum Optics. Springer Series in Synergetics. Springer, 2004.

[16] C. W. Gardiner. Quantum Noise. Springer-Verlag, 1991.

[17] Roy Glauber". The quantum theory of optical coherence. Physical Review, 130(6):25292539, 1963.

[18] Roy J Glauber. Coherent and incoherent states of the radiation field. Physical Review, 131(6):2766, 1963.

[19] Gilbert Grynberg, Alain Aspect, and Claude Fabre. Introduction to quantum optics: from the semi-classical approach to quantized light. Cambridge university press, 2010.

[20] Hermann A. Haus. Waves and fields in optoeletronics. Prentice-Hall, Inc., Englewood Cliffs, New Jersey 07632, 1984.

[21] N. Imoto". Quantum nondemolition measurement of the photon number via the optical kerr effect. Physical Review A, 32(4):2287-2292, 1985.

[22] JD Jackson. 1975classical electrodynamics. Wiley, New York., 1962.

[23] NG Van Kampen. Stochastic Processes in Physics and Chemistry, Third Edition (NorthHolland Personal Library). North Holland, 3 edition, 2007.

[24] H. J. Kimble and D. F. Walls. Squeezed states of the electromagnetic field: Introduction to feature issue. J. Opt. Soc. Am. B, 4(10):1449, Oct 1987.

[25] M. Levenson. Generation and detection of squeezed states of light by nondegenerate fourwave mixing in an optical fiber. Physical Review A, 32(3):1550-1562, 1985.

[26] M. D. Levenson, R. M. Shelby, and S. H. Perlmutter. Squeezing of classical noise by nondegenerate four-wave mixing in an optical fiber. Opt. Lett., 10(10):514-516, Oct 1985.

[27] M. D. Levenson, R. M. Shelby, M. Reid, and D. F. Walls. Quantum nondemolition detection of optical quadrature amplitudes. Phys. Rev. Lett., 57:2473-2476, Nov 1986. 
[28] MD Levenson and RM Shelby. Experimentalists' difficulties in optical squeezed state generation. In Laser Spectroscopy VII, pages 250-253. Springer, 1985.

[29] Jacob S Levy, Alexander Gondarenko, Mark A Foster, Amy C Turner-Foster, Alexander L Gaeta, and Michal Lipson. Cmos-compatible multiple-wavelength oscillator for on-chip optical interconnects. Nature Photonics, 4(1):37-40, 2009.

[30] William H. Louisell. Quantum Statistical Properties of Radiation. John Wiley and Sons, 1973.

[31] LA Lugiato and G Strini. On the squeezing obtainable in parametric oscillators and bistable absorption. Optics Communications, 41(1):67-70, 1982.

[32] S. S. Kano M. D. Levenson. Introduction to nonlinear optics. Academic Press, 1988.

[33] Leonard Mandel and Emil Wolf. Coherence properties of optical fields. Reviews of modern physics, 37(2):231, 1965.

[34] Leonard Mandel and Emil Wolf. Optical coherence and quantum optics. Cambridge university press, 1995.

[35] Michel E. Marhic. Fiber Optical Parametric Amplifiers, Oscillators and Related Devices. Cambridge University Press, New York, NY, USA, 1st edition, 2007.

[36] M Martinelli, CL Garrido Alzar, PH Souto Ribeiro, and P Nussenzveig. Classical and quantum properties of optical parametric oscillators. Brazilian journal of physics, 31(4):597-615, 2001.

[37] Marcelo Martinelli. Compressão de Ruído Quântico e Efeitos Transversos em Osciladores Paramétricos Óticos. PhD thesis, Universidade de São Paulo, 2002.

[38] Piort Myslinski, Czeslaw Szubert, Allan J Bruce, David J DiGiovanni, and Bera Palsdottir. Performance of high-concentration erbium-doped fiber amplifiers. Photonics Technology Letters, IEEE, 11(8):973-975, 1999.

[39] Z. Y. Ou, S. F. Pereira, H. J. Kimble, and K. C. Peng. Realization of the einstein-podolskyrosen paradox for continuous variables. Phys. Rev. Lett., 68:3663-3666, Jun 1992.

[40] Wolfgang P. Schleich. Quantum optics in phase space. WILEY-VCH, 1st edition edition, 2001.

[41] Bonny L Schumaker. Noise in homodyne detection. Optics letters, 9(5):189-191, 1984. 
[42] Marlan O Scully, M Suhail Zubairy, and Ian A Walmsley. Quantum optics. American Journal of Physics, 67:648, 1999.

[43] R. Shelby. Generation of squeezed states of light with a fiber-optic ring interferometer. Physical Review A, 33(6):4008-4025, 1986.

[44] R. M. Shelby, M. D. Levenson, S. H. Perlmutter, R. G. DeVoe, and D. F. Walls. Broadband parametric deamplification of quantum noise in an optical fiber. Phys. Rev. Lett., 57:691-694, Aug 1986.

[45] RM Shelby, MD Levenson, and PW Bayer. Guided acoustic-wave brillouin scattering. Physical Review B, 31(8):5244, 1985.

[46] EK Sittig and GA Coquin. Visualization of plane-strain vibration modes of a long cylinder capable of producing sound radiation. The Journal of the Acoustical Society of America, 48:1150, 1970.

[47] R_E Slusher, LW Hollberg, Bernard Yurke, JC Mertz, and JF Valley. Observation of squeezed states generated by four-wave mixing in an optical cavity. Physical Review Letters, $55(22): 2409,1985$.

[48] Richard G Smith. Optical power handling capacity of low loss optical fibers as determined by stimulated raman and brillouin scattering. Applied Optics, 11(11):2489-2494, 1972.

[49] R.H. Stolen, J.E. Bjorkholm, and A. Ashkin. Phase matched three wave mixing in silica fiber optical waveguides. Applied Physics Letters, 24(7):308-310, 1974.

[50] RH Stolen and EP Ippen. Raman gain in glass optical waveguides. Applied Physics Letters, 22(6):276-278, 1973.

[51] Ro N Thurston. Elastic waves in rods and clad rods. The Journal of the Acoustical Society of America, 64:1, 1978.

[52] A. S. Villar, L. S. Cruz, K. N. Cassemiro, M. Martinelli, and P. Nussenzveig. Generation of bright two-color continuous variable entanglement. Phys. Rev. Lett., 95:243603, Dec 2005.

[53] Alessandro S Villar. The conversion of phase to amplitude fluctuations of a light beam by an optical cavity. American Journal of Physics, 76:922, 2008.

[54] Daniel F Walls. Squeezed states of light. nature, 306:141-146, 1983.

[55] DF Walls and GJ Milburn. Quantum Optics. Springer, 1995.

[56] Yariv. Quantum Electronics. A. Wiley, New York, 1989. 
[57] Horace P Yuen and Vincent WS Chan. Noise in homodyne and heterodyne detection. Optics Letters, 8(3):177-179, 1983.

[58] Bernard Yurke. Use of cavities in squeezed-state generation. Physical Review A, 29(1):408410, 1984.

[59] T-C Zhang, J-Ph Poizat, P Grelu, J-F Roch, Ph Grangier, F Marin, A Bramati, V Jost, MD Levenson, and E Giacobino. Quantum noise of free-running and externally-stabilized laser diodes. Quantum and Semiclassical Optics: Journal of the European Optical Society Part B, 7(4):601, 1995.

[60] Pavel Zhuravlev. Development of a Stable Laser Lock System. PhD thesis, 2012. 